\title{
Combining Very Large Quadratic and Cubic Nonlinear Optical Responses in Extended, Tris-Chelate Metallochromophores with Six $\pi$-Conjugated Pyridinium Substituents
}

\author{
Benjamin J. Coe, ${ }^{*, \dagger}$ John Fielden, ${ }^{\dagger}$ Simon P. Foxon, ${ }^{\dagger}$ Bruce S. Brunschwig, ${ }^{\neq}$ \\ Inge Asselberghs, ${ }^{\S}$ Koen Clays, ${ }^{\S}$ Anna Samoc," and Marek Samoc ${ }^{\prime l, \perp}$ \\ School of Chemistry, University of Manchester, Oxford Road, Manchester M13 9PL, U.K., \\ Molecular Materials Research Center, Beckman Institute, MC 139-74, California Institute of \\ Technology, 1200 East California Boulevard, Pasadena, California 91125, Department of \\ Chemistry, University of Leuven, Celestijnenlaan 200D, B-3001 Leuven, Belgium, Laser Physics \\ Centre, Research School of Physics and Engineering, Australian National University, \\ Canberra, Australian Capital Territory 0200, Australia, and Institute of Physical and \\ Theoretical Chemistry, Wroctaw University of Technology, \\ Wybrzeże Wyspiańskiego 27, 50-370 Wrocław, Poland
}

Received December 14, 2009; E-mail: b.coe@manchester.ac.uk

\begin{abstract}
We describe a series of nine new complex salts in which electron-rich Rull or Fe" centers are connected via $\pi$-conjugated bridges to six electron-accepting $\mathrm{N}$-methyl-/ $\mathrm{N}$-arylpyridinium groups. This work builds upon our previous preliminary studies (Coe, B. J. J. Am. Chem. Soc. 2005, 127, 13399-13410; J. Phys. Chem. A 2007, 111, 472-478), with the aims of achieving greatly enhanced NLO properties and also combining large quadratic and cubic effects in potentially redox-switchable molecules. Characterization has involved various techniques, including electronic absorption spectroscopy and cyclic voltammetry. The complexes display intense, visible $d \rightarrow \pi^{*}$ metal-to-ligand charge-transfer (MLCT) bands, and their $\pi \rightarrow \pi^{\star}$ intraligand charge-transfer (ILCT) absorptions in the near-UV region show molar extinction coefficients as high as ca. $3.5 \times 10^{5} \mathrm{M}^{-1} \mathrm{~cm}^{-1}$. Molecular quadratic nonlinear optical (NLO) responses $\beta$ have been determined by using hyper-Rayleigh scattering at 800 and $1064 \mathrm{~nm}$ and also via Stark (electroabsorption) spectroscopic studies. The directly and indirectly derived $\beta$ values are very large, with the Stark-based static first hyperpolarizabilities $\beta_{0}$ reaching as high as ca. $10^{-27}$ esu, and generally increase on extending the $\pi$-conjugation and enhancing the electron-accepting strength of the ligands. Cubic NLO properties have also been measured by using the Z-scan technique, revealing relatively high two-photon absorption cross sections of up to $2500 \mathrm{GM}$ at $750 \mathrm{~nm}$.
\end{abstract}

\section{Introduction}

The promise of diverse applications, including optical data processing and biological imaging, has stimulated many studies with organic nonlinear optical (NLO) materials over recent years. ${ }^{1}$ For example, NLO effects allow the manipulation of laser light beams and may thus form the basis of all-optical computing devices. Although purely organic compounds have received most attention, transition metal coordination and

†niversity of Manchester.

California Institute of Technology.

$\S$ University of Leuven.

"Australian National University.

${ }^{\perp}$ Wrocław University of Technology.

(1) (a) Zyss, J. Molecular Nonlinear Optics: Materials, Physics and Devices; Academic Press: Boston, 1994. (b) Bosshard, Ch.; Sutter, K.; Prêtre, Ph.; Hulliger, J.; Flörsheimer, M.; Kaatz, P.; Günter, P. Organic Nonlinear Optical Materials; Gordon \& Breach: Amsterdam, The Netherlands, 1995; Advances in Nonlinear Optics Vol. 1. (c) Nonlinear Optics of Organic Molecules and Polymers; Nalwa, H. S., Miyata, S., Eds.; CRC Press: Boca Raton, FL, 1997. (d) Marder, S. R. Chem. Commun. 2006, 131-134. (e) Nonlinear Optical Properties of Matter: From Molecules to Condensed Phases; Papadopoulos, M. G., Leszczynski, J., Sadlej, A. J., Eds.; Springer: Dordrecht, The Netherlands, 2006 organometallic complexes are especially interesting in this context. Such compounds offer unparalleled structural diversity and scope for the creation of multifunctional optical materials, ${ }^{2}$ featuring for example redox-switchable NLO responses. ${ }^{3}$

Most NLO chromophores comprise relatively simple dipolar electronic structures, but octupolar compounds have also attracted considerable interest, especially for potential quadratic (second-order) applications. ${ }^{4}$ Octupolar molecules have zero net ground-state dipole moments, but large dipole moment changes accompanying intramolecular charge-transfer (ICT) excitations can lead to substantial values of the first hyperpolarizability $\beta$, which governs quadratic NLO effects at the molecular level. Molecules with large two-photon absorption (2PA) cross sections $\sigma_{2}$ are of major interest for a wide range of potential applications, including optical power limiting, two-photon upconversion lasing, 3D optical data storage, and photodynamic cancer therapy. ${ }^{5}$ The quantity $\sigma_{2}$ is related to the imaginary part of the second hyperpolarizability $\gamma$, and the latter is the origin of cubic NLO responses. It is established that large $\gamma$ and $\sigma_{2}$ values require extended conjugated $\pi$-systems, and recent studies 
have featured various branched structures, including octupolar chromophores. ${ }^{6}$

Zyss et al. first highlighted the octupolar nature of $D_{3}$ trischelate transition metal complexes, using hyper-Rayleigh scattering (HRS) measurements and a three-state theoretical model to derive reasonably large static first hyperpolarizabilities $\beta_{0}$ of ca. $50 \times 10^{-30}$ esu for the salts $\left[\mathrm{Ru}^{\mathrm{II}}(\mathrm{bpy})_{3}\right] \mathrm{Br}_{2}\left(\mathrm{bpy}=2,2^{\prime}-\right.$ bipyridyl) and $\left[\mathrm{Ru}^{\mathrm{II}}(\text { phen })_{3}\right] \mathrm{Cl}_{2}$ (phen $=1,10$-phenanthroline). ${ }^{7}$ An off-resonance $\beta_{0}$ response is the quantity that is of most relevance to quadratic NLO applications, because these normally require the avoidance of any actual light absorption. Later HRS studies on $\mathrm{a}\left[\mathrm{Ru}^{\mathrm{II}}(\mathrm{bpy})_{3}\right]^{2+}$ derivative with six electron-donating styryl substituents revealed a huge $\beta_{0}$ value of $2200 \times 10^{-30}$

(2) (a) Kanis, D. R.; Ratner, M. A.; Marks, T. J. Chem. Rev. 1994, 94, 195-242. (b) Long, N. J. Angew. Chem., Int. Ed. Engl. 1995, 34, 2138. (c) Whittall, I. R.; McDonagh, A. M.; Humphrey, M. G.; Samoc, M. Adv. Organomet. Chem. 1998, 42, 291-362. (d) Whittall, I. R.; McDonagh, A. M.; Humphrey, M. G.; Samoc, M. Adv. Organomet. Chem. 1998, 43, 349-405. (e) Heck, J.; Dabek, S.; Meyer-Friedrichsen, T.; Wong, H. Coord. Chem. Rev. 1999, 190-192, 1217-1254. (f) Gray, G. M.; Lawson, C. M. In Optoelectronic Properties of Inorganic Compounds; Roundhill, D. M., Fackler, J. P., Jr., Eds.; Plenum: New York, 1999; pp 1-27. (g) Shi, S. In Optoelectronic Properties of Inorganic Compounds; Roundhill, D. M., Fackler, J. P., Jr., Eds.; Plenum: New York, 1999; pp 55-105. (h) Le Bozec, H.; Renouard, T. Eur. J. Inorg. Chem. 2000, 229-239. (i) Barlow, S.; Marder, S. R. Chem. Commun. 2000, 1555-1562. (j) Lacroix, P. G. Eur. J. Inorg. Chem. 2001, 339-348. (k) Di Bella, S. Chem. Soc. Rev. 2001, 30, 355-366. (1) Goovaerts, E.: Wenseleers, W. E.: Garcia, M. H.: Cross, G. H. In Handbook of Advanced Electronic and Photonic Materials and Devices; Nalwa, H. S., Ed.; Academic Press: San Diego, CA, 2001; Vol. 9, pp 127-191. (m) Coe, B. J. In Comprehensive Coordination Chemistry II; McCleverty, J. A., Meyer, T. J., Eds.; Elsevier Pergamon: Oxford, U.K., 2004; Vol. 9, pp 621-687. (n) Maury, O.; Le Bozec, H. Acc. Chem. Res. 2005, 38, 691-704. (o) Cariati, E.; Pizzotti, M.; Roberto, D.; Tessore, F.; Ugo, R. Coord. Chem. Rev. 2006, 250, 1210-1233. (p) Coe, B. J. Acc. Chem. Res. 2006, 39, 383-393. (q) Thompson, M. E.; Djurovich, P. E.; Barlow, S.; Marder, S. In Comprehensive Organometallic Chemistry III; Crabtree, R. H., Mingos, D. M. P., Eds.; Elsevier: Oxford, U.K., 2006; Vol. 12, pp. 101-194. (r) Zhang, C.; Song, Y.-L.; Wang, X. Coord. Chem. Rev. 2007, 251, 111-141. (s) Andraud, C.; Maury, O. Eur. J. Inorg. Chem. 2009, 4357-4371.

(3) (a) Coe, B. J.; Houbrechts, S.; Asselberghs, I.; Persoons, A. Angew. Chem., Int. Ed. 1999, 38, 366-369. (b) Weyland, T.; Ledoux, I.; Brasselet, S.; Zyss, J.; Lapinte, C. Organometallics 2000, 19, 52355237. (c) Malaun, M.; Reeves, Z. R.; Paul, R. L.; Jeffery, J. C.; McCleverty, J. A.; Ward, M. D.; Asselberghs, I.; Clays, K.; Persoons, A. Chem. Commun. 2001, 49-50. (d) Malaun, M.; Kowallick, R.; McDonagh, A. M.; Marcaccio, M.; Paul, R. L.; Asselberghs, I.; Clays, K.; Persoons, A.; Bildstein, B.; Fiorini, C.; Nunzi, J.-M.; Ward, M. D.; McCleverty, J. A. Dalton Trans. 2001, 3025-3038. (e) Cifuentes, M. P.; Powell, C. E.; Humphrey, M. G.; Heath, G. A.; Samoc, M.; Luther-Davies, B. J. Phys. Chem. A 2001, 105, 9625-9627. (f) Paul, F.; Costuas, K.; Ledoux, I.; Deveau, S.; Zyss, J.; Halet, J.-F.; Lapinte, C. Organometallics 2002, 21, 5229-5235. (g) Powell, C. E.; Cifuentes, M. P.; Morrall, J. P.; Stranger, R.; Humphrey, M. G.; Samoc, M.; Luther-Davies, B.; Heath, G. A. J. Am. Chem. Soc. 2003, 125, 602610. (h) Asselberghs, I.; Clays, K.; Persoons, A.; McDonagh, A. M.; Ward, M. D.; McCleverty, J. A. Chem. Phvs. Lett. 2003, 368, 408411. (i) Powell, C. E.; Humphrey, M. G.; Cifuentes, M. P.; Morrall, J. P.; Samoc, M.; Luther-Davies, B. J. Phvs. Chem. A 2003, 107, 11264-11266. (j) Sporer, C.; Ratera, I.; Ruiz-Molina, D.; Zhao, Y.X.; Vidal-Gancedo, J.; Wurst, K.; Jaitner, P.; Clays, K.; Persoons, A.; Rovira, C.; Veciana, J. Angew. Chem., Int. Ed. 2004, 43, 52665268. (k) Cifuentes, M. P.; Powell, C. E.; Morrall, J. P.; McDonagh, A. M.; Lucas, N. T.; Humphrey, M. G.; Samoc, M.; Houbrechts, S.; Asselberghs, I.; Clays, K.; Persoons, A.; Isoshima, T. J. Am. Chem. Soc. 2006, 128, 10819-10832. (1) Samoc, M.; Gauthier, N.; Cifuentes, M. P.; Paul, F.; Lapinte, C.; Humphrey, M. G. Angew. Chem., Int. Ed. 2006, 45, 7376-7379. (m) Dalton, G. T.; Cifuentes, M. P.; Petrie, S.; Stranger, R.; Humphrey, M. G.; Samoc, M. J. Am. Chem. Soc. Clays, K.; Foerier, S.; Verbiest, T.; Asselberghs, I. J. Am. Chem. Soc. 2008, 130, 3286-3287. (o) Wahab, A.; Bhattacharya, M.; Ghosh, S.; Samuelson, A. G.; Das, P. K. J. Phvs. Chem. B 2008, 112, 28422847. esu. $^{8}$ However, other workers found that this $\beta_{0}$ value is overestimated due to contributions from $2 \mathrm{PA}$-induced luminescence, ${ }^{9}$ and subsequent investigations have thus given a smaller (albeit still large) $\beta_{0}$ value of $380 \times 10^{-30}$ esu for this complex. ${ }^{10}$ A number of other quadratic NLO studies have been carried out with derivatives of $\left[\mathrm{Ru}^{\mathrm{II}}(\mathrm{bpy})_{3}\right]^{2+}$ and related complexes of other metals. ${ }^{2 n, 10,11}$ Very recent reports concerning such chromophores have also focused on their 2PA properties, from both a largely experimental perspective ${ }^{12}$ and purely theoretical analyses. $^{13}$

(4) Selected examples: (a) Ledoux, I.; Zyss, J.; Siegel, J. S.; Brienne, J.; Lehn, J.-M. Chem. Phys. Lett. 1990, 172, 440-444. (b) Joffre, M.; Yaron, D.; Silbey, R. J.; Zyss, J. J. Chem. Phvs. 1992, 97, 56075615. (c) Verbiest, T.; Clays, K.; Persoons, A.; Meyers, F.; Brédas, J. L. Opt. Lett. 1993, 18, 525-527. (d) Zyss, J. J. Chem. Phys. 1993, 98, 6583-6599. (e) Verbiest, T.; Clays, K.; Samyn, C.; Wolff, J.; Reinhoudt, D.; Persoons, A. J. Am. Chem. Soc. 1994, 116, 93209323. (f) Zyss, J.; Ledoux, I. Chem. Rev. 1994, 94, 77-105. (g) Wolff, J. J.; Wortmann, R. Adv. Phvs. Org. Chem. 1999, 32, 121-217. (h) Wolff, J. J.; Siegler, F.; Matschiner, R.; Wortmann, R. Angew. Chem.. Int. Ed. 2000, 39, 1436-1439. (i) Cho, B. R.; Piao, M. J.; Son, K. H.; Lee, S. H.; Yoon, S. J.; Jeon, S.-J.; Cho, M.-H. Chem. Eur. J. 2002, 8, 3907-3916. (j) Brunel, J.; Mongin, O.; Jutand, A.; Ledoux, I.; Zyss, J.; Blanchard-Desce, M. Chem. Mater. 2003, 15, 4139-4148. (k) Cui, Y. Z.; Fang, Q.; Lei, H.; Xue, G.; Yu, W. T. Chem. Phvs. Lett. 2003, 377, 507-511. (1) Ray, P. C.; Leszczynski, J. Chem. Phvs. Lett. 2004, 399, 162-166. (m) Claessens, C. G.; González-Rodríguez, D.; Torres, T.; Martín, G.; Agulló-Lopez, F.; Ledoux, I.; Zyss, J.; Ferro, V. R.; García de la Vega, J. M. J. Phys. Chem. B 2005, 109, 3800-3806. (n) Le Floch, V.; Brasselet, S.; Zyss, J.; Cho, B. R.; Lee, S. H.; Jeon, S.-J.; Cho, M.-H.; Min, K. S.; Suh, M. P. Adv. Mater. 2005, 17, 196200. (o) Jeong, M.-Y.; Kim, H. M.; Jeon, S.-J.; Brasselet, S.; Cho, B. R. Adv. Mater. 2007, 19, 2107-2111. (p) Liu, Y.; Xu, X.; Zheng, F.; Cui, Y. Angew. Chem., Int. Ed. 2008, 47, 4538-4541. (q) Kim, H. M.; Cho, B. R. J. Mater. Chem. 2009, 19, 7402-7409.

(5) Selected examples: (a) Wang, C.; Wang, X.-M.; Shao, Z.-S.; Zhao, X.; Zhou, G.-Y.; Wang, D.; Fang, Q.; Jiang, M.-H. Appl. Opt. 2001, 40, 2475-2478. (b) Zheng, Q.-D.; He, G. S.; Lin, T.-C.; Prasad, P. N. J. Mater. Chem. 2003, 13, 2499-2504. (c) He, G. S.; Lin, T.-C.; Prasad, P. N.; Cho, C.-C.; Yu, L.-J. Appl. Phys. Lett. 2003, 82, 4717-4719. (d) Yang, Z.; Wu, Z.-K.; Ma, J.-S.; Xia, A.-D.; Li, Q.-S.; Liu, C.-L.; Gong, Q.-H. Appl. Phys. Lett. 2005, 86, 061903. (e) Samoc, M.; Morrall, J. P.; Dalton, G. T.; Cifuentes, M. P.; Humphrey, M. G. Angew. Chem., Int. Ed. 2007, 46, 731-733. (f) Terenziani, F.; Katan, C.; Badaeva, E.; Tretiak, S.; Blanchard-Desce, M. Adv. Mater. 2008, 20, 4641-4678. (g) Jana, A.; Jang, S. Y.; Shin, J.-Y.; De, A. K.; Goswami, D.; Kim, D.; Bharadwaj, P. J. Chem. Eur. J. 2008, 14, 10628-10638. (h) D’Aléo, A.; Picot, A.; Baldeck, P. L.; Andraud, C.; Maury, O. Inorg. Chem. 2008, 47, 10269-10279. (i) He, G. S.; Tan, L.-S.; Zheng, Q.-D.; Prasad, P. N. Chem. Rev. 2008, 108, 12451330. (j) Collings, J. C.; Poon, S.-Y.; Le Droumaguet, C.; Charlot, M.; Katan, C.; Pålsson, L.-O.; Beeby, A.; Mosely, J. A.; Kaiser, H. M.; Kaufmann, D.; Wong, W.-Y.; Blanchard-Desce, M.; Marder, T. B. Chem. Eur. J. 2009, 15, 198-208. (k) Pawlicki, M.; Collins, H. A.; Denning, R. G.; Anderson, H. L. Angew. Chem., Int. Ed. 2009, 48, 3244-3266. (1) Dvornikov, A. S.; Walker, E. P.; Rentzepis, P. M. $\underline{J}$ Phvs. Chem. A 2009, 113, 13633-13644.

(6) Selected examples: (a) Lee, W.-H.; Lee, H.-C.; Kim, J.-A.; Choi, J.H.; Cho, M.-H.; Jeon, S.-J.; Cho, B. R. J. Am. Chem. Soc. 2001, 123, 10658-10667. (b) Beljonne, D.; Wenseleers, W.; Zojer, E.; Shuai, Z.G.; Vogel, H.; Pond, S. J. K.; Perry, J. W.; Marder, S. R.; Bredas, J.-L. Adv. Funct. Mater. 2002, 12, 631-641. (c) Kannan, R.; He, G. S.; Lin, T.-C.; Prasad, P. N.; Vaia, R. A.; Tan, L.-S. Chem. Mater. 2004, 16, 185-194. (d) Ray, P. C.; Leszczynski, J. J. Phys. Chem. A 2005, 109, 6689-6696. (e) Zhou, X.; Feng, J.-K.; Ren, A.-M. Chem. Phvs. Lett. 2005, 403, 7-15. (f) Easwaramoorthi, S.; Shin, J.-Y.; Cho, S.; Kim, P.-S.; Inokuma, Y.; Tsurumaki, E.; Osuka, A.; Kim, D.-H. Chem. Eur. J. 2009, 15, 12005-12017. (g) Kim, H. M.; Seo, M. S.; Jeon, S.-J.; Cho, B. R. Chem. Commun. 2009, 7422-7424.

(7) Zyss, J.; Dhenaut, C.; Chauvan, T.; Ledoux, I. Chem. Phys. Lett. 1993, 206, 409-414.

(8) Dhenaut, C.; Ledoux, I.; Samuel, I. D. W.; Zyss, J.; Bourgault, M.; Le Bozec, H. Nature 1995, 374, 339-342.

(9) Morrison, I. D.; Denning, R. G.; Laidlaw, W. M.; Stammers, M. A. Rev. Sci. Instrum. 1996, 67, 1445-1453.

(10) Le Bozec, H.; Renouard, T.; Bourgault, M.; Dhenaut, C.; Brasselet, S.; Ledoux, I.; Zyss, J. Synth. Met. 2001, 124, 185-189. 
While $\left[\mathrm{M}^{\mathrm{II}}(\mathrm{bpy})_{3}\right]^{2+}(\mathrm{M}=\mathrm{Fe}, \mathrm{Ru}, \mathrm{Zn}$, etc. $)$ complex units themselves have octupolar ground states, they form dipolar ICT excited states. Polarized HRS and Stark spectroscopic measurements have shown that the $\beta$ responses of $\left[\mathrm{Ru}^{\mathrm{II}}(\mathrm{bpy})_{3}\right]^{2+}$ derivatives hexasubstituted with electron-donating styryl groups are dominated by multiple degenerate dipolar intraligand chargetransfer (ILCT) excitations, rather than an octupolar transition. ${ }^{14}$ These ILCT transitions are red-shifted by metal coordination and are directionally opposed to the metal-to-ligand chargetransfer (MLCT) excitations. ${ }^{10,14}$ Since all previous NLO studies with this class of tris-chelate complexes have involved electrondonor-substituted species, ${ }^{2 n, 10-14}$ we recently prepared a series of $\left[\mathrm{M}^{\mathrm{II}}(\mathrm{bpy})_{3}\right]^{2+}(\mathrm{M}=\mathrm{Fe}, \mathrm{Ru})$ derivatives bearing electronwithdrawing pyridinium groups and carried out quadratic ${ }^{15}$ and cubic $^{16}$ NLO investigations. In these complexes, the MLCT transitions are expected to dominate the NLO responses because the ILCT processes, despite also being directed toward the pyridinium units, occur at very short wavelengths (below about $330 \mathrm{~nm}$ ). The present report concerns extended relatives of our previously reported chromophores, with the aim being to achieve greatly enhanced quadratic and cubic NLO properties. The contributions of the ILCT transitions to the $\beta$ responses become more significant in these new complexes and are even amenable to assessment by Stark spectroscopy in two cases.

\section{Experimental Section}

Materials and Procedures. The compounds cis- $\mathrm{Ru}^{\mathrm{II}} \mathrm{Cl}_{2}$ (DMSO) ${ }_{4},{ }^{17} 4,4^{\prime}$-bis[(diethoxyphosphinyl)methyl]-2,2'-bipyridyl, ${ }^{18}$ 2,2'-bipyridyl-4,4'-dicarboxaldehyde, ${ }^{19} N$-phenyl-4-picolinium chloride hydrate $\left(\left[\mathrm{Phpic}^{+}\right] \mathrm{Cl} \cdot 1.25 \mathrm{H}_{2} \mathrm{O}\right),{ }^{20}(E)$-3-(4-pyridyl)-2-prope-

(11) Selected examples: (a) Le Bouder, T.; Maury, O.; Le Bozec, H.; Ledoux, I.; Zyss, J. Chem. Commun. 2001, 2430-2431. (b) Le Bozec, H.; Le Bouder, T.; Maury, O.; Bondon, A.; Ledoux, I.; Deveau, S.; Zyss, J. Adv. Mater. 2001, 13, 1677-1681. (c) Sénéchal, K.; Maury, O.; Le Bozec, H.; Ledoux, I.; Zyss, J. J. Am. Chem. Soc. 2002, 124, 4560-4561. (d) Le Bouder, T.; Maury, O.; Bondon, O.; Costuas, K.; Amouyal, E.; Ledoux, I.; Zyss, J.; Le Bozec, H. J. Am. Chem. Soc. 2003, 125, 12284-12299. (e) Viau, L.; Bidault, S.; Maury, O.; Brasselet, S.; Ledoux, I.; Zyss, J.; Ishow, E.; Nakatani, K.; Le Bozec, H. J. Am. Chem. Soc. 2004, 126, 8386-8387. (f) Maury, O.; Viau, L.; Sénéchal, K.; Corre, B.; Guégan, J.-P.; Renouard, T.; Ledoux, I.; Zyss, J.; Le Bozec, H. Chem. Eur. J. 2004, 10, 4454-4466. (g) Bidault, S.; Brasselet, S.; Zyss, J.; Maury, O.; Le Bozec, H. J. Chem. Phvs. 2007, 126, 034312-1-034312-13.

(12) (a) Girardot, C.; Lemercier, G.; Mulatier, J.-C.; Chauvin, J.; Baldeck, P. L.; Andraud, C. Dalton Trans. 2007, 3421-3426. (b) Feuvrie, C.; Maury, O.; Le Bozec, H.; Ledoux, I.; Morrall, J. P.; Dalton, G. T.; Samoc, M.; Humphrey, M. G. J. Phvs. Chem. A 2007, 111, 89808985. (c) Boca, S. C.; Four, M.; Bonne, A.; van der Sanden, B.; Astilean, S.; Baldeck, P. L.; Lemercier, G. Chem. Commun. 2009, $4590-4592$

(13) (a) Liu, X.-J.; Feng, J.-K.; Ren, A.-M.; Cheng, H.; Zhou, X. J. Chem. Phvs. 2004, 120, 11493-11499. (b) Zhang, X.-B.; Feng, J.-K.; Ren, A.-M. J. Phvs. Chem. A 2007, 111, 1328-1338.

(14) Vance, F. W.; Hupp, J. T. J. Am. Chem. Soc. 1999, 121, 4047-4053.

(15) Coe, B. J.; Harris, J. A.; Brunschwig, B. S.; Asselberghs, I.; Clays, K.; Garín, J.; Orduna, J. J. Am. Chem. Soc. 2005, 127, 13399-13410.

(16) Coe, B. J.; Samoc, M.; Samoc, A.; Zhu, L.-Y.; Yi, Y.-P.; Shuai, Z.G. J. Phys. Chem. A 2007, 111, 472-478.

(17) Evans, I. P.; Spencer, A.; Wilkinson, G. J. Chem. Soc., Dalton Trans. 1973, 204-209.

(18) Gillaizeau-Gauthier, I.; Odobel, F.; Alebbi, M.; Argazzi, R.; Costa, E.; Bignozzi, C. A.; Qu, P.; Meyer, G. J. Inorg. Chem. 2001, 40, $6073-$ 6079.

(19) Maury, O.; Guégan, J.-P.; Renouard, T.; Hilton, A.; Dupau, P.; Sandon, N.; Toupet, L.; Le Bozec, H. New J. Chem. 2001, 25, 1553-1566.

(20) Coe, B. J.; Harris, J. A.; Asselberghs, I.; Clays, K.; Olbrechts, G.; Persoons, A.; Hupp, J. T.; Johnson, R. C.; Coles, S. J.; Hursthouse, M. B.; Nakatani, K. Adv. Funct. Mater. 2002, 12, 110-116. nal, ${ }^{21}$ and 4-[(E)-2-(4-pyridyl)vinyl]benzaldehyde ${ }^{22}$ were prepared according to published procedures. All other reagents were obtained commercially and used as supplied. Products were dried overnight in a vacuum desiccator $\left(\mathrm{CaSO}_{4}\right)$ prior to characterization.

General Physical Measurements. ${ }^{1} \mathrm{H}$ NMR spectra were recorded on a Bruker $400 \mathrm{MHz}$ spectrometer, and all shifts are quoted with respect to TMS. The fine splitting of pyridyl or phenyl ring $\mathrm{AA}^{\prime} \mathrm{BB}^{\prime}$ patterns is ignored, and the signals are reported as simple doublets, with $J$ values referring to the two most intense peaks. Elemental analyses were performed by the Microanalytical Laboratory, University of Manchester, using a Carlo Erba EA1108 instrument. UV - vis spectra were obtained by using a Shimadzu UV-2401 PC spectrophotometer, and mass spectra were recorded by using electron impact on a Micromass Trio 2000 or positive electrospray on a Micromass Platform II spectrometer. Luminescence spectra were recorded on a Gilden photonics fluoroSENS fluorimeter. Cyclic voltammetric measurements were performed by using an EG\&G PAR Model 283 potentiostat/galvanostat. A single-compartment cell was used with a silver/silver chloride reference electrode ( $3 \mathrm{M} \mathrm{NaCl}$, saturated $\mathrm{AgCl}$ ) separated by a salt bridge from a 2 $\mathrm{mm}$ glassy-carbon-disk working electrode and Pt-wire auxiliary electrode. Acetonitrile was freshly distilled (from $\mathrm{CaH}_{2}$ ), and $\left[\mathrm{N}\left(\mathrm{C}_{4} \mathrm{H}_{9}-n\right)_{4}\right] \mathrm{PF}_{6}$, as supplied from Fluka, was used as the supporting electrolyte. Solutions containing ca. $10^{-3} \mathrm{M}$ analyte $(0.1 \mathrm{M}$ electrolyte) were deaerated by purging with $\mathrm{N}_{2}$. All $E_{1 / 2}$ values were calculated from $\left(E_{\mathrm{pa}}+E_{\mathrm{pc}}\right) / 2$ at a scan rate of $200 \mathrm{mV} \mathrm{s}^{-1}$.

Synthesis of 4,4'-Bis[(E)-2-(4-pyridyl)vinyl]-2,2'-bipyridyl, bbpe. Potassium tert-butoxide $(624 \mathrm{mg}, 5.56 \mathrm{mmol})$ was added to a stirred solution of 4,4'-bis-[(diethoxyphosphinyl)methyl]-2,2'-bipyridyl $(1.00 \mathrm{~g}, 2.19 \mathrm{mmol})$ and 4-pyridinecarboxaldehyde (528 mg, 4.93 $\mathrm{mmol})$ in tetrahydrofuran $(40 \mathrm{~mL})$. The reaction vessel was sealed and protected from the light, and the contents were stirred at room temperature for $2 \mathrm{~h}$. Distilled water $(60 \mathrm{~mL})$ was added, and the reaction mixture was stirred for a further $2 \mathrm{~min}$. The cream-colored solid was filtered off, washed with copious amounts of water followed by diethyl ether, and dried: yield $681 \mathrm{mg}, 84 \% .{ }^{1} \mathrm{H}$ NMR $\delta_{\mathrm{H}}\left(\mathrm{CDCl}_{3}\right): 8.72\left(2 \mathrm{H}, \mathrm{d}, J=5.1 \mathrm{~Hz}, \mathrm{C}_{5} \mathrm{H}_{3} \mathrm{~N}\right), 8.64(4 \mathrm{H}, \mathrm{d}, J=$ $\left.6.1 \mathrm{~Hz}, \mathrm{C}_{5} \mathrm{H}_{4} \mathrm{~N}\right), 8.60\left(2 \mathrm{H}, \mathrm{s}, \mathrm{C}_{5} \mathrm{H}_{3} \mathrm{~N}\right), 7.45-7.41\left(6 \mathrm{H}, \mathrm{C}_{5} \mathrm{H}_{3} \mathrm{~N}+\right.$ $\left.\mathrm{C}_{5} \mathrm{H}_{4} \mathrm{~N}\right), 7.39(2 \mathrm{H}, \mathrm{d}, J=16.5 \mathrm{~Hz}, \mathrm{CH}), 7.32(2 \mathrm{H}, \mathrm{d}, J=16.4$ $\mathrm{Hz}, \mathrm{CH}$ ). Anal. Calcd for $\mathrm{C}_{24} \mathrm{H}_{18} \mathrm{~N}_{4} \cdot 0.5 \mathrm{H}_{2} \mathrm{O}: \mathrm{C}, 77.61 ; \mathrm{H}, 5.16 ; \mathrm{N}$, 15.08. Found: C, 77.80; H, 4.89; N, 15.05. EI-MS: $\mathrm{m} / \mathrm{z} 363$ $\left([\mathrm{MH}]^{+}\right)$.

Synthesis of 4,4'-Bis[(E)-2-(N-methyl-4-pyridyl)vinyl]-2,2'-bipyridyl Iodide, $\left[\mathbf{M e}_{2} \mathbf{b b p e}^{2+}\right] \mathbf{I}_{2}$. A mixture of bbpe $\cdot 0.5 \mathrm{H}_{2} \mathrm{O}(303 \mathrm{mg}$, $0.816 \mathrm{mmol})$ and iodomethane $(10 \mathrm{~mL})$ in acetone $(40 \mathrm{~mL})$ was heated at reflux in the dark for $4 \mathrm{~h}$. The orange precipitate was filtered off, washed with a small amount of acetone followed by copious amounts of $\mathrm{CH}_{2} \mathrm{Cl}_{2}$ and then diethyl ether, and dried: yield $454 \mathrm{mg}, 84 \%$. ${ }^{1} \mathrm{H}$ NMR $\delta_{\mathrm{H}}\left(\mathrm{CD}_{3} \mathrm{SOCD}_{3}\right): 8.98(4 \mathrm{H}, \mathrm{d}, J=6.8$ $\left.\mathrm{Hz}, \mathrm{C}_{5} \mathrm{H}_{4} \mathrm{~N}\right), 8.86\left(2 \mathrm{H}, \mathrm{d}, J=5.2 \mathrm{~Hz}, \mathrm{C}_{5} \mathrm{H}_{3} \mathrm{~N}\right), 8.74(2 \mathrm{H}, \mathrm{s}$, $\left.\mathrm{C}_{5} \mathrm{H}_{3} \mathrm{~N}\right), 8.37\left(4 \mathrm{H}, \mathrm{d}, J=6.8 \mathrm{~Hz}, \mathrm{C}_{5} \mathrm{H}_{4} \mathrm{~N}\right), 8.16(2 \mathrm{H}, \mathrm{d}, J=16.4$ $\mathrm{Hz}, \mathrm{CH}), 7.91(2 \mathrm{H}, \mathrm{d}, J=16.4 \mathrm{~Hz}, \mathrm{CH}), 7.81(2 \mathrm{H}, \mathrm{dd}, J=5.0$, $\left.1.4 \mathrm{~Hz}, \mathrm{C}_{5} \mathrm{H}_{3} \mathrm{~N}\right), 4.30(6 \mathrm{H}, \mathrm{s}, \mathrm{Me})$. Anal. Calcd for $\mathrm{C}_{26} \mathrm{H}_{24} \mathrm{I}_{2} \mathrm{~N}_{4} \cdot 0.7 \mathrm{H}_{2} \mathrm{O}: \mathrm{C}, 47.39 ; \mathrm{H}, 3.89 ; \mathrm{N}, 8.50$. Found: $\mathrm{C}, 47.44$; H, 3.61; N, 8.45. ES-MS: $m / z 519\left([\mathrm{M}-\mathrm{I}]^{+}\right)$.

Synthesis of 4,4'-Bis[(E)-2-(N-methyl-4-pyridyl)vinyl]-2,2'-bipyridyl Hexafluorophosphate, $\left[\mathrm{Me}_{2} \mathrm{bbpe}^{2+}\right]\left[\mathrm{PF}_{6}\right]_{2} \cdot\left[\mathrm{Me}_{2} \mathrm{bbpe}^{2+}\right] \mathrm{I}_{2}$ • $0.7 \mathrm{H}_{2} \mathrm{O}$ (446 mg, $0.677 \mathrm{mmol}$ ) was dissolved in $2 / 1$ methanol/water. Slow addition of aqueous $\mathrm{NH}_{4} \mathrm{PF}_{6}(0.6 \mathrm{M})$ followed by water $(50$ $\mathrm{mL}$ ) gave a white precipitate, which was filtered off, washed with copious amounts of water and then diethyl ether, and dried: yield $433 \mathrm{mg}, 92 \%$. ${ }^{1} \mathrm{H}$ NMR $\delta_{\mathrm{H}}\left(\mathrm{CD}_{3} \mathrm{SOCD}_{3}\right): 8.97(4 \mathrm{H}, \mathrm{d}, J=6.6$ $\left.\mathrm{Hz}, \mathrm{C}_{5} \mathrm{H}_{4} \mathrm{~N}\right), 8.86\left(2 \mathrm{H}, \mathrm{d}, J=5.0 \mathrm{~Hz}, \mathrm{C}_{5} \mathrm{H}_{3} \mathrm{~N}\right), 8.75(2 \mathrm{H}, \mathrm{s}$,

(21) Coe, B. J.; Jones, L. A.; Harris, J. A.; Brunschwig, B. S.; Asselberghs, I.; Clays, K.; Persoons, A.; Garín, J.; Orduna, J. J. Am. Chem. Soc 2004, 126, 3880-3891.

(22) Ichimura, K.; Watanabe, S. J. Polvm. Sci., Polvm. Chem. 1982, 20, 1419-1432. 
$\left.\mathrm{C}_{5} \mathrm{H}_{3} \mathrm{~N}\right), 8.35\left(4 \mathrm{H}, \mathrm{d}, J=6.5 \mathrm{~Hz}, \mathrm{C}_{5} \mathrm{H}_{4} \mathrm{~N}\right), 8.14(2 \mathrm{H}, \mathrm{d}, J=16.4$ $\mathrm{Hz}, \mathrm{CH}), 7.90(2 \mathrm{H}, \mathrm{d}, J=16.5 \mathrm{~Hz}, \mathrm{CH}), 7.80(2 \mathrm{H}, \mathrm{dd}, J=5.2$, $\left.1.4 \mathrm{~Hz}, \mathrm{C}_{5} \mathrm{H}_{3} \mathrm{~N}\right), 4.30(6 \mathrm{H}, \mathrm{s}, \mathrm{Me})$. Anal. Calcd for $\mathrm{C}_{26} \mathrm{H}_{24} \mathrm{~F}_{12} \mathrm{~N}_{4} \mathrm{P}_{2} \cdot 0.8 \mathrm{H}_{2} \mathrm{O}: \mathrm{C}, 44.81 ; \mathrm{H}, 3.70 ; \mathrm{N}, 8.04$. Found: $\mathrm{C}, 44.79$; H, 3.44; N, 8.10. ES-MS: $m / z, 537\left(\left[\mathrm{M}-\mathrm{PF}_{6}\right]^{+}\right)$.

Synthesis of $4,4^{\prime}$-Bis $\{(E)-2-[N-(2,4-d i n i t r o p h e n y l)-4-p y r i d y l]$ vinyl $\}-2,2^{\prime}$-bipyridyl Chloride, $\left[(2,4-\mathrm{DNPh})_{2} \mathrm{bbpe}^{2+}\right] \mathrm{Cl}_{2}$. A mixture of bbpe $\cdot 0.5 \mathrm{H}_{2} \mathrm{O}(300 \mathrm{mg}, 0.808 \mathrm{mmol})$ and 2,4-dinitrochlorobenzene $(1.81 \mathrm{~g}, 8.94 \mathrm{mmol})$ in ethanol $(40 \mathrm{~mL})$ was heated at reflux for $72 \mathrm{~h}$, producing a beige precipitate. The precipitate was filtered off, washed with a small amount of ethanol and then dichloromethane and diethyl ether, and dried: yield $546 \mathrm{mg}, 81 \% .{ }^{1} \mathrm{H}$ NMR $\delta_{\mathrm{H}}\left(\mathrm{CD}_{3} \mathrm{SOCD}_{3}\right): 9.38\left(4 \mathrm{H}, \mathrm{d}, J=6.9 \mathrm{~Hz}, \mathrm{C}_{5} \mathrm{H}_{4} \mathrm{~N}\right), 9.15(2$ $\left.\mathrm{H}, \mathrm{d}, J=2.6 \mathrm{~Hz}, \mathrm{C}_{6} \mathrm{H}_{3}\right), 9.00\left(2 \mathrm{H}, \mathrm{dd}, J=8.7,2.5 \mathrm{~Hz}, \mathrm{C}_{6} \mathrm{H}_{3}\right)$, $8.92\left(2 \mathrm{H}, \mathrm{d}, J=5.0 \mathrm{~Hz}, \mathrm{C}_{5} \mathrm{H}_{3} \mathrm{~N}\right), 8.83\left(2 \mathrm{H}, \mathrm{s}, \mathrm{C}_{5} \mathrm{H}_{3} \mathrm{~N}\right), 8.67(4 \mathrm{H}$, $\left.\mathrm{d}, J=6.9 \mathrm{~Hz}, \mathrm{C}_{5} \mathrm{H}_{4} \mathrm{~N}\right), 8.47-8.41\left(4 \mathrm{H}, \mathrm{C}_{6} \mathrm{H}_{3}+\mathrm{CH}\right), 8.11(2 \mathrm{H}$, $\mathrm{d}, J=16.4 \mathrm{~Hz}, \mathrm{CH}), 7.89\left(2 \mathrm{H}, \mathrm{dd}, J=5.0,1.3 \mathrm{~Hz}, \mathrm{C}_{5} \mathrm{H}_{3} \mathrm{~N}\right)$. Anal. Calcd for $\mathrm{C}_{36} \mathrm{H}_{24} \mathrm{Cl}_{2} \mathrm{~N}_{8} \mathrm{O}_{8} \cdot 3.8 \mathrm{H}_{2} \mathrm{O}: \mathrm{C}, 51.72 ; \mathrm{H}, 3.81 ; \mathrm{N}$, 13.40. Found: C, 52.14; H, 3.62; N, 12.90. ES-MS: $m / z, 731$ ([M $\left.-\mathrm{Cl}]^{+}\right), 348\left([\mathrm{M}-2 \mathrm{Cl}]^{2+}\right)$.

Synthesis of $4,4^{\prime}$-Bis $\{(E)-2-[N$-(2,4-dinitrophenyl)-4-pyridyl $]$ vinyl\}-2,2'-bipyridyl Hexafluorophosphate, [(2,4-DNPh $\left.)_{2} \mathbf{b b p e}^{2+}\right]$ $\left[\mathbf{P F}_{6}\right]_{2} \cdot\left[(2,4-\mathrm{DNPh})_{2} \mathrm{bbpe}^{2+}\right] \mathrm{Cl}_{2} \cdot 3.8 \mathrm{H}_{2} \mathrm{O}(536 \mathrm{mg}, 0.641 \mathrm{mmol})$ was dissolved in a $1 / 1$ methanol/water mixture. Slow addition of aqueous $\mathrm{NH}_{4} \mathrm{PF}_{6}(0.6 \mathrm{M})$ gave a beige precipitate, which was filtered off, washed with water, and dried: yield $570 \mathrm{mg}, 89 \%$. ${ }^{1} \mathrm{H}$ NMR $\delta_{\mathrm{H}}$ $\left(\mathrm{CD}_{3} \mathrm{SOCD}_{3}\right): 9.34\left(4 \mathrm{H}, \mathrm{d}, J=6.7 \mathrm{~Hz}, \mathrm{C}_{5} \mathrm{H}_{4} \mathrm{~N}\right), 9.15(2 \mathrm{H}, \mathrm{d}, J=$ $\left.2.5 \mathrm{~Hz}, \mathrm{C}_{6} \mathrm{H}_{3}\right), 9.00\left(2 \mathrm{H}, \mathrm{dd}, J=8.7,2.4 \mathrm{~Hz}, \mathrm{C}_{6} \mathrm{H}_{3}\right), 8.92(2 \mathrm{H}, \mathrm{d}$, $\left.J=5.0 \mathrm{~Hz}, \mathrm{C}_{5} \mathrm{H}_{3} \mathrm{~N}\right), 8.83\left(2 \mathrm{H}, \mathrm{s}, \mathrm{C}_{5} \mathrm{H}_{3} \mathrm{~N}\right), 8.63(4 \mathrm{H}, \mathrm{d}, J=6.6$ $\left.\mathrm{Hz}, \mathrm{C}_{5} \mathrm{H}_{4} \mathrm{~N}\right), 8.43\left(2 \mathrm{H}, \mathrm{d}, J=8.7 \mathrm{~Hz}, \mathrm{C}_{6} \mathrm{H}_{3}\right), 8.38(2 \mathrm{H}, \mathrm{d}, J=$ $16.6 \mathrm{~Hz}, \mathrm{CH}), 8.07(2 \mathrm{H}, \mathrm{d}, J=16.4 \mathrm{~Hz}, \mathrm{CH}), 7.87(2 \mathrm{H}, \mathrm{d}, J=$ $5.3 \mathrm{~Hz}, \mathrm{C}_{5} \mathrm{H}_{3} \mathrm{~N}$ ). Anal. Calcd for $\mathrm{C}_{36} \mathrm{H}_{24} \mathrm{~F}_{12} \mathrm{~N}_{8} \mathrm{O}_{8} \mathrm{P}_{2} \cdot \mathrm{H}_{2} \mathrm{O}$ : C, 43.04; H, 2.61; N, 11.15. Found: C, 43.24; H, 2.31; N, 10.96. ES-MS: $m / z 841\left(\left[\mathrm{M}-\mathrm{PF}_{6}\right]^{+}\right), 348\left(\left[\mathrm{M}-2 \mathrm{PF}_{6}\right]^{2+}\right)$.

Synthesis of 4,4'-Bis[(E)-2-( $N$-phenyl-4-pyridyl)vinyl]-2,2' -bipyridyl Chloride, $\left[\mathbf{P h}_{2} \mathbf{b b p e}^{2+}\right] \mathbf{C l}_{2}$. A mixture of $2,2^{\prime}$-bipyridyl-4,4'dicarboxaldehyde $(350 \mathrm{mg}, 1.65 \mathrm{mmol})$ and $\left[\mathrm{Phpic}^{+}\right] \mathrm{Cl} \cdot 1.25 \mathrm{H}_{2} \mathrm{O}$ $(746 \mathrm{mg}, 3.27 \mathrm{mmol})$ in acetic anhydride $(20 \mathrm{~mL})$ was heated at reflux in the dark for $1.5 \mathrm{~h}$. The resulting beige precipitate was filtered off, washed with a small amount of acetic anhydride followed by acetone and then diethyl ether, and dried. Further purification was effected by reprecipitation from DMF/diethyl ether: yield $540 \mathrm{mg}, 49 \% .{ }^{1} \mathrm{H}$ NMR $\delta_{\mathrm{H}}\left(\mathrm{CD}_{3} \mathrm{SOCD}_{3}\right): 9.37(4 \mathrm{H}, \mathrm{d}, J=$ $\left.6.4 \mathrm{~Hz}, \mathrm{C}_{5} \mathrm{H}_{4} \mathrm{~N}\right), 8.90\left(2 \mathrm{H}, \mathrm{d}, J=5.0 \mathrm{~Hz}, \mathrm{C}_{5} \mathrm{H}_{3} \mathrm{~N}\right), 8.80(2 \mathrm{H}, \mathrm{s}$, $\left.\mathrm{C}_{5} \mathrm{H}_{3} \mathrm{~N}\right), 8.56\left(4 \mathrm{H}, \mathrm{d}, J=6.5 \mathrm{~Hz}, \mathrm{C}_{5} \mathrm{H}_{4} \mathrm{~N}\right), 8.37(2 \mathrm{H}, \mathrm{d}, J=16.2$ $\mathrm{Hz}, \mathrm{CH}), 8.08(2 \mathrm{H}, \mathrm{d}, J=16.4 \mathrm{~Hz}, \mathrm{CH}), 7.95-7.92(4 \mathrm{H}, \mathrm{m}, \mathrm{Ph})$, $7.87\left(2 \mathrm{H}, \mathrm{d}, J=5.8 \mathrm{~Hz}, \mathrm{C}_{5} \mathrm{H}_{3} \mathrm{~N}\right), 7.80-7.72(6 \mathrm{H}, \mathrm{Ph})$. Anal. Calcd for $\mathrm{C}_{36} \mathrm{H}_{28} \mathrm{Cl}_{2} \mathrm{~N}_{4} \cdot 4.5 \mathrm{H}_{2} \mathrm{O}$ : C, 64.67; $\mathrm{H}, 5.58 ; \mathrm{N}, 8.38$. Found: C, 64.71; H, 5.46; N, 8.49. ES-MS: $m / z 551\left([\mathrm{M}-\mathrm{Cl}]^{+}\right), 258([\mathrm{M}$ $\left.-2 \mathrm{Cl}]^{2+}\right)$.

Synthesis of 4,4'-Bis[(E)-2-( $N$-phenyl-4-pyridyl)vinyl]-2,2'-bipyridyl Hexafluorophosphate, $\left[\mathrm{Ph}_{2} \mathbf{b b p e}^{2+}\right]\left[\mathrm{PF}_{\mathbf{6}}\right]_{2} \cdot\left[\mathrm{Ph}_{2} \mathrm{bbpe}^{2+}\right] \mathrm{Cl}_{2}$ • $4.5 \mathrm{H}_{2} \mathrm{O}(540 \mathrm{mg}, 0.808 \mathrm{mmol})$ was dissolved in methanol. Slow addition of aqueous $\mathrm{NH}_{4} \mathrm{PF}_{6}(0.6 \mathrm{M})$ gave a beige precipitate, which was filtered off, washed with water and then diethyl ether, and dried. Further purification was effected by reprecipitation from DMF/ diethyl ether: yield $612 \mathrm{mg}, 93 \% .{ }^{1} \mathrm{H}$ NMR $\delta_{\mathrm{H}}\left(\mathrm{CD}_{3} \mathrm{SOCD}_{3}\right): 9.35$ $\left(4 \mathrm{H}, \mathrm{d}, J=6.6 \mathrm{~Hz}, \mathrm{C}_{5} \mathrm{H}_{4} \mathrm{~N}\right), 8.90\left(2 \mathrm{H}, \mathrm{d}, J=4.9 \mathrm{~Hz}, \mathrm{C}_{5} \mathrm{H}_{3} \mathrm{~N}\right)$, $8.81\left(2 \mathrm{H}, \mathrm{s}, \mathrm{C}_{5} \mathrm{H}_{3} \mathrm{~N}\right), 8.53\left(4 \mathrm{H}, \mathrm{d}, J=6.6 \mathrm{~Hz}, \mathrm{C}_{5} \mathrm{H}_{4} \mathrm{~N}\right), 8.34(2 \mathrm{H}$, $\mathrm{d}, J=16.7 \mathrm{~Hz}, \mathrm{CH}), 8.05(2 \mathrm{H}, \mathrm{d}, J=16.5 \mathrm{~Hz}, \mathrm{CH}), 7.93-7.91$ $(4 \mathrm{H}, \mathrm{m}, \mathrm{Ph}), 7.85\left(2 \mathrm{H}, \mathrm{d}, J=4.4 \mathrm{~Hz}, \mathrm{C}_{5} \mathrm{H}_{3} \mathrm{~N}\right), 7.80-7.74(6 \mathrm{H}$, $\mathrm{Ph}$ ). Anal. Calcd for $\mathrm{C}_{36} \mathrm{H}_{28} \mathrm{~F}_{12} \mathrm{~N}_{4} \mathrm{P}_{2} \cdot 0.6 \mathrm{H}_{2} \mathrm{O}: \mathrm{C}, 52.90 ; \mathrm{H}, 3.60 ; \mathrm{N}$, 6.85. Found: C, 52.90; H, 3.16; N, 6.85. ES-MS: $m / z 661$ ([M $\left.\mathrm{PF}_{6}\right]^{+}$).

Synthesis of $4,4^{\prime}$-Bis $[(E, E)-4-(4-p y r i d y l) b u t a-1,3-d i e n y l]-2$, $2^{\prime}$-bipyridyl, bbpb. This compound was prepared in a manner similar to that for bbpe by using potassium tert-butoxide $(312 \mathrm{mg}$, $2.78 \mathrm{mmol}), 4,4^{\prime}$-bis[(diethoxyphosphinyl)methyl]-2,2'-bipyridyl (500 mg, $1.10 \mathrm{mmol}$ ), and (E)-3-(4-pyridyl)-2-propenal (321 mg,
$2.41 \mathrm{mmol})$ instead of 4-pyridinecarboxaldehyde. Distilled water $(60 \mathrm{~mL})$ was added and a cream-colored solid obtained: yield 325 mg, 70\%. ${ }^{1} \mathrm{H}$ NMR $\delta_{\mathrm{H}}\left(\mathrm{CDCl}_{3}\right): 8.67\left(2 \mathrm{H}, \mathrm{d}, J=5.5 \mathrm{~Hz}, \mathrm{C}_{5} \mathrm{H}_{3} \mathrm{~N}\right)$, $8.59\left(4 \mathrm{H}, \mathrm{d}, J=6.1 \mathrm{~Hz}, \mathrm{C}_{5} \mathrm{H}_{4} \mathrm{~N}\right), 8.49\left(2 \mathrm{H}, \mathrm{s}, \mathrm{C}_{5} \mathrm{H}_{3} \mathrm{~N}\right), 7.36-7.25$ $\left(8 \mathrm{H}, \mathrm{C}_{5} \mathrm{H}_{3} \mathrm{~N}+\mathrm{C}_{5} \mathrm{H}_{4} \mathrm{~N}+\mathrm{CH}\right), 7.16(2 \mathrm{H}, \mathrm{dd}, J=15.2,10.8 \mathrm{~Hz}$, $\mathrm{CH}), 6.80(2 \mathrm{H}, \mathrm{d}, J=15.4 \mathrm{~Hz}, \mathrm{CH}), 6.72(2 \mathrm{H}, \mathrm{d}, J=15.4 \mathrm{~Hz}$, $\mathrm{CH}$ ). Anal. Calcd for $\mathrm{C}_{28} \mathrm{H}_{22} \mathrm{~N}_{4} \cdot 0.5 \mathrm{H}_{2} \mathrm{O}: \mathrm{C}, 79.41 ; \mathrm{H}, 5.47 ; \mathrm{N}$, 13.23. Found: C, 79.43; H, 5.57; N, 12.79. EI-MS: $m / z 415\left(\mathbf{M}^{+}\right)$.

Synthesis of $4,4^{\prime}-\operatorname{Bis}[(E, E)-4-(N$-methyl-4-pyridyl)buta-1, 3-dienyl]-2,2'-bipyridyl Iodide, $\left[\mathbf{M e}_{2} \mathbf{b b p b}^{2+}\right] \mathbf{I}_{2}$. This compound was prepared in a manner similar to that for $\left[\mathrm{Me}_{2} \mathrm{bbpe}^{2+}\right] \mathrm{I}_{2}$ by using bbpb $0.5 \mathrm{H}_{2} \mathrm{O}(200 \mathrm{mg}, 0.472 \mathrm{mmol})$ instead of bbpe $\cdot 0.5 \mathrm{H}_{2} \mathrm{O}$ and acetone $(50 \mathrm{~mL})$. Further purification was effected by precipitation from 1/1 DMF/diethyl ether to give an orange solid: yield $224 \mathrm{mg}$, 65\%. ${ }^{1} \mathrm{H} \mathrm{NMR} \delta_{\mathrm{H}}\left(\mathrm{CD}_{3} \mathrm{SOCD}_{3}\right): 8.87\left(4 \mathrm{H}, \mathrm{d}, J=6.9 \mathrm{~Hz}, \mathrm{C}_{5} \mathrm{H}_{4} \mathrm{~N}\right)$, $8.75\left(2 \mathrm{H}, \mathrm{d}, J=5.1 \mathrm{~Hz}, \mathrm{C}_{5} \mathrm{H}_{3} \mathrm{~N}\right), 8.54\left(2 \mathrm{H}, \mathrm{s}, \mathrm{C}_{5} \mathrm{H}_{3} \mathrm{~N}\right), 8.21(4 \mathrm{H}$, $\left.\mathrm{d}, J=6.9 \mathrm{~Hz}, \mathrm{C}_{5} \mathrm{H}_{4} \mathrm{~N}\right), 7.86(2 \mathrm{H}, \mathrm{dd}, J=15.4,10.8 \mathrm{~Hz}, \mathrm{CH})$, $7.72\left(2 \mathrm{H}, \mathrm{dd}, J=5.2,1.6 \mathrm{~Hz}, \mathrm{C}_{5} \mathrm{H}_{3} \mathrm{~N}\right), 7.62(2 \mathrm{H}, \mathrm{dd}, J=15.5$, $10.8 \mathrm{~Hz}, \mathrm{CH}), 7.19(2 \mathrm{H}, \mathrm{d}, J=15.5 \mathrm{~Hz}, \mathrm{CH}), 7.12(2 \mathrm{H}, \mathrm{d}, J=$ $15.5 \mathrm{~Hz}, \mathrm{CH}), 4.26(6 \mathrm{H}, \mathrm{s}, \mathrm{Me})$. Anal. Calcd for $\mathrm{C}_{30} \mathrm{H}_{28} \mathrm{I}_{2} \mathrm{~N}_{4} \cdot 1.9 \mathrm{H}_{2} \mathrm{O}: \mathrm{C}, 49.18 ; \mathrm{H}, 4.38 ; \mathrm{N}, 7.65$. Found: C, 49.17; $\mathrm{H}, 4.06 ; \mathrm{N}, 7.56$. ES-MS: $m / z$ z $571\left([\mathrm{M}-\mathrm{I}]^{+}\right)$.

Synthesis of 4,4'-Bis[(E,E)-4-(N-methyl-4-pyridyl)buta-1,3-dienyl]-2,2'-bipyridyl Hexafluorophosphate, $\left[\mathrm{Me}_{2} \mathbf{b b p b}^{2+}\right]\left[\mathrm{PF}_{6}\right]_{2}$. This compound was prepared in a manner similar to that for $\left[\mathrm{Me}_{2} \mathrm{bbpe}^{2+}\right]\left[\mathrm{PF}_{6}\right]_{2}$ by using $\left[\mathrm{Me}_{2} \mathrm{bbpb}^{2+}\right] \mathrm{I}_{2} \cdot 1.9 \mathrm{H}_{2} \mathrm{O}(216 \mathrm{mg}, 0.295$ $\mathrm{mmol})$ instead of $\left[\mathrm{Me}_{2} \mathrm{bbpe}^{2+}\right] \mathrm{I}_{2} \cdot 0.7 \mathrm{H}_{2} \mathrm{O}$ in DMF $(30 \mathrm{~mL})$ to afford a bright yellow solid: yield $193 \mathrm{mg}, 85 \%$. ${ }^{1} \mathrm{H}$ NMR $\delta_{\mathrm{H}}$ $\left(\mathrm{CD}_{3} \mathrm{SOCD}_{3}\right): 8.86\left(4 \mathrm{H}, \mathrm{d}, J=6.9 \mathrm{~Hz}, \mathrm{C}_{5} \mathrm{H}_{4} \mathrm{~N}\right), 8.75(2 \mathrm{H}, \mathrm{d}, J=$ $\left.5.1 \mathrm{~Hz}, \mathrm{C}_{5} \mathrm{H}_{3} \mathrm{~N}\right), 8.55\left(2 \mathrm{H}, \mathrm{s}, \mathrm{C}_{5} \mathrm{H}_{3} \mathrm{~N}\right), 8.20(4 \mathrm{H}, \mathrm{d}, J=7.0 \mathrm{~Hz}$, $\left.\mathrm{C}_{5} \mathrm{H}_{4} \mathrm{~N}\right), 7.85(2 \mathrm{H}, \mathrm{dd}, J=15.4,10.7 \mathrm{~Hz}, \mathrm{CH}), 7.71(2 \mathrm{H}, \mathrm{dd}, J$ $\left.=5.4,1.6 \mathrm{~Hz}, \mathrm{C}_{5} \mathrm{H}_{3} \mathrm{~N}\right), 7.62(2 \mathrm{H}, \mathrm{dd}, J=15.5,10.7 \mathrm{~Hz}, \mathrm{CH})$, $7.18(2 \mathrm{H}, \mathrm{d}, J=15.5 \mathrm{~Hz}, \mathrm{CH}), 7.11(2 \mathrm{H}, \mathrm{d}, J=15.5 \mathrm{~Hz}, \mathrm{CH})$, 4.26 (6 H, s, Me). Anal. Calcd for $\mathrm{C}_{30} \mathrm{H}_{28} \mathrm{~F}_{12} \mathrm{~N}_{4} \mathrm{P}_{2} \cdot 1.8 \mathrm{H}_{2} \mathrm{O}$ : C, 46.98; H, 4.15; N, 7.31. Found: C, 47.00; H, 3.83; N, 7.28. ES-MS: $\mathrm{m} / \mathrm{z}$ $589\left(\left[\mathrm{M}-\mathrm{PF}_{6}\right]^{+}\right)$.

Synthesis of 4,4'-Bis $((E, E)-2-\{4-[2-(4-p y r i d y l) v i n y l] p h e n y l\}$ vinyl)-2,2'-bipyridyl, bbpvb. This compound was prepared in a manner similar to that for bbpb by using 4-[(E)-2-(4-pyridyl)vinyl]benzaldehyde (515 mg, $2.45 \mathrm{mmol}$ ) instead of (E)-3-(4-pyridyl)2-propenal in tetrahydrofuran $(30 \mathrm{~mL})$. Distilled water $(40 \mathrm{~mL})$ was added and a cream-colored solid obtained after an additional wash with ethyl acetate: yield $325 \mathrm{mg}, 51 \%$. ${ }^{1} \mathrm{H} \mathrm{NMR} \delta_{\mathrm{H}}$ $\left(\mathrm{CF}_{3} \mathrm{CO}_{2} \mathrm{D}\right): 9.58\left(2 \mathrm{H}, \mathrm{d}, J=6.4 \mathrm{~Hz}, \mathrm{C}_{5} \mathrm{H}_{3} \mathrm{~N}\right), 9.31\left(2 \mathrm{H}, \mathrm{s}, \mathrm{C}_{5} \mathrm{H}_{3} \mathrm{~N}\right)$, $9.27\left(4 \mathrm{H}, \mathrm{d}, J=6.7 \mathrm{~Hz}, \mathrm{C}_{5} \mathrm{H}_{4} \mathrm{~N}\right), 8.99\left(2 \mathrm{H}, \mathrm{d}, J=6.4 \mathrm{~Hz}, \mathrm{C}_{5} \mathrm{H}_{3} \mathrm{~N}\right)$, $8.78\left(4 \mathrm{H}, \mathrm{d}, J=6.8 \mathrm{~Hz}, \mathrm{C}_{5} \mathrm{H}_{4} \mathrm{~N}\right), 8.68(2 \mathrm{H}, \mathrm{d}, J=16.2 \mathrm{~Hz}, \mathrm{CH})$, $8.52-8.45\left(10 \mathrm{H}, \mathrm{C}_{6} \mathrm{H}_{4}+\mathrm{CH}\right), 8.17(2 \mathrm{H}, \mathrm{d}, J=16.1 \mathrm{~Hz}, \mathrm{CH})$, $8.07(2 \mathrm{H}, \mathrm{d}, J=16.2 \mathrm{~Hz}, \mathrm{CH})$. Anal. Calcd for $\mathrm{C}_{40} \mathrm{H}_{30} \mathrm{~N}_{4} \cdot 0.5 \mathrm{H}_{2} \mathrm{O}$ : C, 83.45; H, 5.43; N, 9.73. Found: C, 83.47; H, 5.20; N, 9.63. EIMS: $m / z, 567\left(\mathrm{M}^{+}\right)$.

Synthesis of 4,4'-Bis((E,E)-2-\{4-[2-(N-methyl-4-pyridyl)vinyl]phenyl\}vinyl)-2,2'-bipyridyl Iodide, $\left[\mathrm{Me}_{2} \mathbf{b b p v b}^{2+}\right] \mathbf{I}_{2}$. This compound was prepared in a manner similar to that for $\left[\mathrm{Me}_{2} \mathrm{bbpb}^{2+}\right] \mathrm{I}_{2}$ by using bbpvb $0.5 \mathrm{H}_{2} \mathrm{O}(200 \mathrm{mg}, 0.347 \mathrm{mmol})$ instead of $\mathrm{bbpb} \cdot 0.5 \mathrm{H}_{2} \mathrm{O}$ to afford an orange solid: yield $447 \mathrm{mg}, 99 \%$. ${ }^{1} \mathrm{H}$ NMR $\delta_{\mathrm{H}}$ $\left(\mathrm{CD}_{3} \mathrm{SOCD}_{3}\right): 8.87\left(4 \mathrm{H}, \mathrm{d}, J=6.9 \mathrm{~Hz}, \mathrm{C}_{5} \mathrm{H}_{4} \mathrm{~N}\right), 8.73(2 \mathrm{H}, \mathrm{d}, J=$ $\left.5.1 \mathrm{~Hz}, \mathrm{C}_{5} \mathrm{H}_{3} \mathrm{~N}\right), 8.61\left(2 \mathrm{H}, \mathrm{s}, \mathrm{C}_{5} \mathrm{H}_{3} \mathrm{~N}\right), 8.23(4 \mathrm{H}, \mathrm{d}, J=6.9 \mathrm{~Hz}$, $\left.\mathrm{C}_{5} \mathrm{H}_{4} \mathrm{~N}\right), 8.04(2 \mathrm{H}, \mathrm{d}, J=16.2 \mathrm{~Hz}, \mathrm{CH}), 7.89-7.81\left(8 \mathrm{H}, \mathrm{m}, \mathrm{C}_{6} \mathrm{H}_{4}\right)$, 7.73-7.68 (4 H, $\left.\mathrm{C}_{5} \mathrm{H}_{3} \mathrm{~N}+\mathrm{CH}\right), 7.61-7.54(4 \mathrm{H}, \mathrm{CH}), 4.26(6 \mathrm{H}$, $\mathrm{s}, \mathrm{Me})$. Anal. Calcd for $\mathrm{C}_{42} \mathrm{H}_{36} \mathrm{I}_{2} \mathrm{~N}_{4} \cdot 2.5 \mathrm{H}_{2} \mathrm{O}: \mathrm{C}, 56.33 ; \mathrm{H}, 4.61 ; \mathrm{N}$, 6.26. Found: C, 56.31; H, 4.29; N, 6.17. ES-MS: $m / z, 723$ ([M $\left.\mathrm{I}]^{+}\right)$.

Synthesis of $4,4^{\prime}-\mathrm{Bis}((E, E)-2-\{4-[2-(N$-methyl-4-pyridyl)vinyl] phenyl\}vinyl)-2,2'-bipyridyl Hexafluorophosphate, $\left[\mathrm{Me}_{2} \mathrm{bbpvb}^{2+}\right]$ $\left[\mathbf{P F}_{6}\right]_{2}$. This compound was prepared in a manner similar to that for $\left[\mathrm{Me}_{2} \mathrm{bbpe}^{2+}\right]\left[\mathrm{PF}_{6}\right]_{2}$ by using $\left[\mathrm{Me}_{2} \mathrm{bbpvb}^{2+}\right] \mathrm{I}_{2} \cdot 2.5 \mathrm{H}_{2} \mathrm{O}(435 \mathrm{mg}$, $0.486 \mathrm{mmol})$ instead of $\left[\mathrm{Me}_{2} \mathrm{bbpe}^{2+}\right] \mathrm{I}_{2} \cdot 0.7 \mathrm{H}_{2} \mathrm{O}$ in DMF $(50 \mathrm{~mL})$. Further purification was effected by precipitation from $1 / 1 \mathrm{DMF} /$ diethyl ether to afford a bright yellow solid: yield $342 \mathrm{mg}, 75 \%$. 
${ }^{1} \mathrm{H} \mathrm{NMR} \delta_{\mathrm{H}}\left(\mathrm{CD}_{3} \mathrm{SOCD}_{3}\right): 8.86\left(4 \mathrm{H}, \mathrm{d}, J=6.7 \mathrm{~Hz}, \mathrm{C}_{5} \mathrm{H}_{4} \mathrm{~N}\right), 8.73$ $\left(2 \mathrm{H}, \mathrm{d}, J=5.1 \mathrm{~Hz}, \mathrm{C}_{5} \mathrm{H}_{3} \mathrm{~N}\right), 8.61\left(2 \mathrm{H}, \mathrm{s}, \mathrm{C}_{5} \mathrm{H}_{3} \mathrm{~N}\right), 8.22(4 \mathrm{H}, \mathrm{d}$, $\left.J=6.8 \mathrm{~Hz}, \mathrm{C}_{5} \mathrm{H}_{4} \mathrm{~N}\right), 8.02(2 \mathrm{H}, \mathrm{d}, J=16.3 \mathrm{~Hz}, \mathrm{CH}), 7.88-7.81$ $\left(8 \mathrm{H}, \mathrm{m}, \mathrm{C}_{6} \mathrm{H}_{4}\right), 7.72-7.68\left(4 \mathrm{H}, \mathrm{C}_{5} \mathrm{H}_{3} \mathrm{~N}+\mathrm{CH}\right), 7.60-7.54(4 \mathrm{H}$, $\mathrm{CH}), 4.26(6 \mathrm{H}, \mathrm{s}, \mathrm{Me})$. Anal. Calcd for $\mathrm{C}_{42} \mathrm{H}_{36} \mathrm{~F}_{12} \mathrm{~N}_{4} \mathrm{P}_{2} \cdot 2.8 \mathrm{H}_{2} \mathrm{O}$ : C, 53.83; H, 4.47; N, 5.98. Found: C, 53.80; H, 3.94; N, 5.97. ESMS: $m / z, 741\left(\left[\mathrm{M}-\mathrm{PF}_{6}\right]^{+}\right)$.

Synthesis of $\left[\mathbf{R u}^{\mathrm{II}}\left(\mathrm{Me}_{2} \mathrm{bbpe}^{2+}\right)_{3}\right]\left[\mathrm{PF}_{6}\right]_{8}$, 2. A mixture of cis$\mathrm{Ru}^{\mathrm{II}} \mathrm{Cl}_{2}$ (DMSO) $)_{4}(33 \mathrm{mg}, 0.068 \mathrm{mmol}$ ), silver(I) tosylate (38 mg, $0.136 \mathrm{mmol})$, and $\left[\mathrm{Me}_{2} \mathrm{bbpe}^{2+}\right]\left[\mathrm{PF}_{6}\right]_{2} \cdot 0.8 \mathrm{H}_{2} \mathrm{O}$ (162 mg, 0.232 $\mathrm{mmol})$ in degassed DMF $(10 \mathrm{~mL})$ was heated at reflux for $4 \mathrm{~h}$ under $\mathrm{Ar}$ in the dark. The resulting red mixture was cooled to room temperature, and the $\mathrm{AgCl}$ precipitate was filtered off. Addition of aqueous $\mathrm{NH}_{4} \mathrm{PF}_{6}$ to the filtrate produced a red precipitate, which was filtered off, washed with water, and dried. The crude product was dissolved in acetonitrile solution, loaded onto a silica gel column $(230-400$ mesh, $5 \times 22 \mathrm{~cm})$, and eluted with $0.1 \mathrm{M}$ $\mathrm{NH}_{4} \mathrm{PF}_{6}$ in acetonitrile, giving a major dark red band. Fractions were collected and compared by TLC. Similar fractions were combined, reduced to dryness, and precipitated from acetone/ aqueous $\mathrm{NH}_{4} \mathrm{PF}_{6}$ to afford a dark red-brown solid: $83 \mathrm{mg}, 49 \%$. ${ }^{1} \mathrm{H}$ NMR $\delta_{\mathrm{H}}\left(\mathrm{CD}_{3} \mathrm{CN}\right): 8.88\left(6 \mathrm{H}, \mathrm{d}, J=1.3 \mathrm{~Hz}, \mathrm{C}_{5} \mathrm{H}_{3} \mathrm{~N}\right), 8.59(12$ $\left.\mathrm{H}, \mathrm{d}, J=6.8 \mathrm{~Hz}, \mathrm{C}_{5} \mathrm{H}_{4} \mathrm{~N}\right), 8.13\left(12 \mathrm{H}, \mathrm{d}, J=6.9 \mathrm{~Hz}, \mathrm{C}_{5} \mathrm{H}_{4} \mathrm{~N}\right)$, 7.90-7.79 $\left(18 \mathrm{H}, \mathrm{CH}+\mathrm{C}_{5} \mathrm{H}_{3} \mathrm{~N}\right), 7.65(6 \mathrm{H}, \mathrm{dd}, J=6.0,1.7 \mathrm{~Hz}$, $\left.\mathrm{C}_{5} \mathrm{H}_{3} \mathrm{~N}\right), \quad 4.27 \quad(18 \mathrm{H}, \quad \mathrm{s}, \quad \mathrm{Me})$. Anal. Calcd for $\mathrm{C}_{78} \mathrm{H}_{72} \mathrm{~F}_{48} \mathrm{~N}_{12} \mathrm{P}_{8} \mathrm{Ru} \cdot 3.5 \mathrm{H}_{2} \mathrm{O}$ : C, 37.45; H, 3.18; N, 6.72. Found: $\mathrm{C}$, 37.44; H, 2.91; N, 6.73.

Synthesis of $\left[\mathbf{R u}^{\mathrm{II}}\left(\mathrm{Me}_{2} \mathrm{bbpe}^{2+}\right)_{3}\right]\left[\mathrm{BPh}_{4}\right]_{7} \mathbf{P F} 6,2 \mathrm{~B}$. A portion of 2 $(19 \mathrm{mg}, 7.60 \mu \mathrm{mol})$ in acetone $(2 \mathrm{~mL})$ was treated with aqueous $\mathrm{NaBPh}_{4}$, and the dark red precipitate was filtered off, washed with water, and dried: yield $22 \mathrm{mg}, 79 \% .{ }^{1} \mathrm{H}$ NMR $\delta_{\mathrm{H}}\left(\mathrm{CD}_{3} \mathrm{SOCD}_{3}\right)$ : $9.23\left(6 \mathrm{H}, \mathrm{s}, \mathrm{C}_{5} \mathrm{H}_{3} \mathrm{~N}\right), 8.89\left(12 \mathrm{H}, \mathrm{d}, J=6.4 \mathrm{~Hz}, \mathrm{C}_{5} \mathrm{H}_{4} \mathrm{~N}\right), 8.16(12$ $\left.\mathrm{H}, \mathrm{d}, J=6.0 \mathrm{~Hz}, \mathrm{C}_{5} \mathrm{H}_{4} \mathrm{~N}\right), 7.99(6 \mathrm{H}, \mathrm{d}, J=16.4 \mathrm{~Hz}, \mathrm{CH}), 7.93$ $(6 \mathrm{H}, \mathrm{d}, J=16.4 \mathrm{~Hz}, \mathrm{CH}), 7.86\left(6 \mathrm{H}, \mathrm{d}, J=6.0 \mathrm{~Hz}, \mathrm{C}_{5} \mathrm{H}_{3} \mathrm{~N}\right), 7.51$ $\left(6 \mathrm{H}, \mathrm{d}, J=6.0, \mathrm{C}_{5} \mathrm{H}_{3} \mathrm{~N}\right), 7.24-7.10(56 \mathrm{H}, \mathrm{m}, \mathrm{Ph}), 6.95-6.84$ $(56 \mathrm{H}, \mathrm{m}, \mathrm{Ph}), 6.80-6.70(28 \mathrm{H}, \mathrm{m}, \mathrm{Ph}), 4.27(18 \mathrm{H}, \mathrm{s}, \mathrm{Me})$. Anal. Calcd for $\mathrm{C}_{246} \mathrm{H}_{212} \mathrm{~B}_{7} \mathrm{~F}_{6} \mathrm{~N}_{12} \mathrm{PRu}$ : C, 80.77; $\mathrm{H}, 5.84 ; \mathrm{N}, 4.59$. Found: C, 80.70; H, 5.76; N, 4.29 .

Synthesis of $\left[\mathbf{R u}^{\mathrm{II}}\left(\mathrm{Ph}_{2} \mathrm{bbpe}^{2+}\right)_{3}\right]\left[\mathbf{P F}_{6}\right]_{8}$, 4. This compound was prepared and purified in a manner similar to that for $\mathbf{2}$ by using $\left[\mathrm{Ph}_{2} \mathrm{bbpe}^{2+}\right]\left[\mathrm{PF}_{6}\right]_{2} \cdot 0.6 \mathrm{H}_{2} \mathrm{O}(192 \mathrm{mg}, 0.235 \mathrm{mmol})$ instead of $\left[\mathrm{Me}_{2} \mathrm{bbpe}^{2+}\right]\left[\mathrm{PF}_{6}\right]_{2} \cdot 0.8 \mathrm{H}_{2} \mathrm{O}$ to afford a dark orange-red solid: yield $124 \mathrm{mg}, 63 \%$. ${ }^{1} \mathrm{H}$ NMR $\delta_{\mathrm{H}}\left(\mathrm{CD}_{3} \mathrm{CN}\right): 9.00(6 \mathrm{H}, \mathrm{d}, J=1.2 \mathrm{~Hz}$, $\left.\mathrm{C}_{5} \mathrm{H}_{3} \mathrm{~N}\right), 8.92\left(12 \mathrm{H}, \mathrm{d}, J=7.1 \mathrm{~Hz}, \mathrm{C}_{5} \mathrm{H}_{4} \mathrm{~N}\right), 8.34(12 \mathrm{H}, \mathrm{d}, J=7.1$ $\left.\mathrm{Hz}, \mathrm{C}_{5} \mathrm{H}_{4} \mathrm{~N}\right), 8.10-7.90\left(18 \mathrm{H}, \mathrm{CH}+\mathrm{C}_{5} \mathrm{H}_{3} \mathrm{~N}\right), 7.77-7.72(36 \mathrm{H}$, $\mathrm{C}_{5} \mathrm{H}_{3} \mathrm{~N}+\mathrm{Ph}$ ). Anal. Calcd for $\mathrm{C}_{108} \mathrm{H}_{84} \mathrm{~F}_{48} \mathrm{~N}_{12} \mathrm{P}_{8} \mathrm{Ru} \cdot 4.3 \mathrm{H}_{2} \mathrm{O}: \mathrm{C}$, 44.91; H, 3.23; N, 5.82. Found: C, 44.91; H, 2.94; N, 5.74.

Synthesis of $\left[\mathbf{R u}^{\mathrm{II}}\left(\mathbf{M e}_{2} \mathbf{b b p b}^{2+}\right)_{3}\right]\left[\mathbf{P F}_{6}\right]_{8}, \mathbf{5}$. This compound was prepared and purified in a manner similar to that for $\mathbf{2}$ by using $\left[\mathrm{Me}_{2} \mathrm{bbpb}^{2+}\right]\left[\mathrm{PF}_{6}\right]_{2} \cdot 1.8 \mathrm{H}_{2} \mathrm{O}(175 \mathrm{mg}, 0.228 \mathrm{mmol})$ instead of $\left[\mathrm{Me}_{2} \mathrm{bbpe}^{2+}\right]\left[\mathrm{PF}_{6}\right]_{2} \cdot 0.8 \mathrm{H}_{2} \mathrm{O}$ to afford a dark orange-red solid: yield $92 \mathrm{mg}, 51 \%$. ${ }^{1} \mathrm{H}$ NMR $\delta_{\mathrm{H}}\left(\mathrm{CD}_{3} \mathrm{CN}\right): 8.70(6 \mathrm{H}, \mathrm{d}, J=1.4 \mathrm{~Hz}$, $\left.\mathrm{C}_{5} \mathrm{H}_{3} \mathrm{~N}\right), 8.48\left(12 \mathrm{H}, \mathrm{d}, J=6.9 \mathrm{~Hz}, \mathrm{C}_{5} \mathrm{H}_{4} \mathrm{~N}\right), 7.99(12 \mathrm{H}, \mathrm{d}, J=6.9$ $\left.\mathrm{Hz}, \mathrm{C}_{5} \mathrm{H}_{4} \mathrm{~N}\right), 7.74\left(6 \mathrm{H}, \mathrm{d}, J=6.1 \mathrm{~Hz}, \mathrm{C}_{5} \mathrm{H}_{3} \mathrm{~N}\right), 7.70-7.59(12 \mathrm{H}$, $\mathrm{CH}), 7.54\left(6 \mathrm{H}, \mathrm{dd}, J=6.1,1.6 \mathrm{~Hz}, \mathrm{C}_{5} \mathrm{H}_{3} \mathrm{~N}\right), 7.16(6 \mathrm{H}, \mathrm{d}, J=$ $14.8 \mathrm{~Hz}, \mathrm{CH}), 7.05(6 \mathrm{H}, \mathrm{d}, J=14.8 \mathrm{~Hz}, \mathrm{CH}), 4.22(18 \mathrm{H}, \mathrm{s}, \mathrm{Me})$. Anal. Calcd for $\mathrm{C}_{90} \mathrm{H}_{84} \mathrm{~F}_{48} \mathrm{~N}_{12} \mathrm{P}_{8} \mathrm{Ru} \cdot 4.3 \mathrm{H}_{2} \mathrm{O}: \mathrm{C}, 40.46 ; \mathrm{H}, 3.49 ; \mathrm{N}$, 6.29. Found: C, 40.46; H, 3.01; N, 6.24.

Synthesis of $\left[\mathbf{R u}^{\mathrm{II}}\left(\mathbf{M e}_{2} \mathbf{b b p v b}{ }^{2+}\right)_{3}\right]\left[\mathbf{P F}_{6}\right]_{8}, 6$. This compound was prepared and purified in a manner similar to that for $\mathbf{2}$ by using cis- $\mathrm{Ru}^{\mathrm{II}} \mathrm{Cl}_{2}(\mathrm{DMSO})_{4}(28 \mathrm{mg}, 0.058 \mathrm{mmol})$, silver(I) tosylate (33 $\mathrm{mg}, 0.118 \mathrm{mmol})$, and $\left[\mathrm{Me}_{2} \mathrm{bbpvb}^{2+}\right]\left[\mathrm{PF}_{6}\right]_{2} \cdot 2.8 \mathrm{H}_{2} \mathrm{O}(180 \mathrm{mg}, 0.203$ mmol) to afford a dark red solid: yield $134 \mathrm{mg}, 75 \%$. ${ }^{1} \mathrm{H}$ NMR $\delta_{\mathrm{H}}$ $\left(\mathrm{CD}_{3} \mathrm{CN}\right): 8.80\left(6 \mathrm{H}, \mathrm{d}, J=1.5 \mathrm{~Hz}, \mathrm{C}_{5} \mathrm{H}_{3} \mathrm{~N}\right), 8.47(12 \mathrm{H}, \mathrm{d}, J=$ $\left.7.0 \mathrm{~Hz}, \mathrm{C}_{5} \mathrm{H}_{4} \mathrm{~N}\right), 8.02\left(12 \mathrm{H}, \mathrm{d}, J=7.0 \mathrm{~Hz}, \mathrm{C}_{5} \mathrm{H}_{4} \mathrm{~N}\right), 7.86-7.77$ $\left(42 \mathrm{H}, \mathrm{C}_{5} \mathrm{H}_{3} \mathrm{~N}+\mathrm{C}_{6} \mathrm{H}_{4}+\mathrm{CH}\right), 7.57(6 \mathrm{H}, \mathrm{dd}, J=6.1,1.5 \mathrm{~Hz}$, $\left.\mathrm{C}_{5} \mathrm{H}_{3} \mathrm{~N}\right), 7.48-7.40(12 \mathrm{H}, \mathrm{CH}), 4.21(18 \mathrm{H}, \mathrm{s}, \mathrm{Me})$. Anal. Calcd for $\mathrm{C}_{126} \mathrm{H}_{108} \mathrm{~F}_{48} \mathrm{~N}_{12} \mathrm{P}_{8} \mathrm{Ru} \cdot 2.3 \mathrm{H}_{2} \mathrm{O}: \mathrm{C}, 48.94 ; \mathrm{H}, 3.67 ; \mathrm{N}, 5.44$. Found: C, 48.93; H, 3.56; N, 5.28.
Synthesis of $\left[\mathrm{Fe}^{\mathrm{II}}\left(\mathrm{Me}_{2} \mathrm{bbpe}^{2+}\right)_{3}\right]\left[\mathrm{PF}_{6}\right]_{8}$, 8. $\mathrm{Fe}^{\mathrm{II}}\left(\mathrm{BF}_{4}\right)_{2} \cdot 6 \mathrm{H}_{2} \mathrm{O}(20$ $\mathrm{mg}, 0.059 \mathrm{mmol})$ was added to a solution of $\left[\mathrm{Me}_{2} \mathrm{bbpe}^{2+}\right]-$ $\left[\mathrm{PF}_{6}\right]_{2} \cdot 0.8 \mathrm{H}_{2} \mathrm{O}(129 \mathrm{mg}, 0.185 \mathrm{mmol})$ in DMF $(10 \mathrm{~mL})$, and the deep blue solution was stirred at room temperature for $2 \mathrm{~h}$ in the dark. Addition of aqueous $\mathrm{NH}_{4} \mathrm{PF}_{6}$ afforded a dark blue precipitate, which was filtered off, washed with water, and dried. Purification was effected as for 2 to afford a dark blue solid: yield $90 \mathrm{mg}, 62 \%$. ${ }^{1} \mathrm{H}$ NMR $\delta_{\mathrm{H}}\left(\mathrm{CD}_{3} \mathrm{CN}\right): 8.90\left(6 \mathrm{H}, \mathrm{d}, J=1.4 \mathrm{~Hz}, \mathrm{C}_{5} \mathrm{H}_{3} \mathrm{~N}\right), 8.59(12$ $\left.\mathrm{H}, \mathrm{d}, J=6.9 \mathrm{~Hz}, \mathrm{C}_{5} \mathrm{H}_{4} \mathrm{~N}\right), 8.14\left(12 \mathrm{H}, \mathrm{d}, J=7.0 \mathrm{~Hz}, \mathrm{C}_{5} \mathrm{H}_{4} \mathrm{~N}\right)$, $7.89(6 \mathrm{H}, \mathrm{d}, J=16.5 \mathrm{~Hz}, \mathrm{CH}), 7.83(6 \mathrm{H}, \mathrm{d}, J=16.5 \mathrm{~Hz}, \mathrm{CH})$, $7.64\left(6 \mathrm{H}, \mathrm{dd}, J=6.1,1.8 \mathrm{~Hz}, \mathrm{C}_{5} \mathrm{H}_{3} \mathrm{~N}\right), 7.57(6 \mathrm{H}, \mathrm{d}, J=6.0 \mathrm{~Hz}$, $\left.\mathrm{C}_{5} \mathrm{H}_{3} \mathrm{~N}\right), \quad 4.29 \quad(18 \mathrm{H}, \quad \mathrm{s}, \quad \mathrm{Me})$. Anal. Calcd for $\mathrm{C}_{78} \mathrm{H}_{72} \mathrm{~F}_{48} \mathrm{FeN}_{12} \mathrm{P}_{8} \cdot 3.5 \mathrm{H}_{2} \mathrm{O}: \mathrm{C}, 38.14 ; \mathrm{H}, 3.24 ; \mathrm{N}, 6.84$. Found: $\mathrm{C}$, 38.13 ; H, 2.89; N, 6.80 .

Synthesis of $\left[\mathrm{Fe}^{\mathrm{II}}\left(\mathrm{Me}_{2} \mathrm{bbpe}^{2+}\right)_{3}\right]\left[\mathrm{BPh}_{4}\right]_{7.3}\left[\mathrm{PF}_{6}\right]_{0.7}, 8 \mathrm{BB}$. This compound was prepared in a manner similar to that for $\mathbf{2 B}$ by using $\mathbf{8}$ (47 mg, 19.1 $\mu \mathrm{mol}$ ) instead of $\mathbf{2}$ to afford a dark blue solid: yield $63 \mathrm{mg}, 90 \% .{ }^{1} \mathrm{H}$ NMR $\delta_{\mathrm{H}}\left(\mathrm{CD}_{3} \mathrm{CN}\right): 8.28\left(6 \mathrm{H}, \mathrm{s}, \mathrm{C}_{5} \mathrm{H}_{3} \mathrm{~N}\right), 8.25$ $\left(12 \mathrm{H}, \mathrm{d}, J=6.8 \mathrm{~Hz}, \mathrm{C}_{5} \mathrm{H}_{4} \mathrm{~N}\right), 7.68\left(12 \mathrm{H}, \mathrm{d}, J=6.8 \mathrm{~Hz}, \mathrm{C}_{5} \mathrm{H}_{4} \mathrm{~N}\right)$, $7.36(6 \mathrm{H}, \mathrm{d}, J=16.4 \mathrm{~Hz}, \mathrm{CH}), 7.25-7.19\left(70.4 \mathrm{H}, \mathrm{C}_{5} \mathrm{H}_{3} \mathrm{~N}+\mathrm{CH}\right.$ $+\mathrm{Ph}), 6.97\left(6 \mathrm{H}, \mathrm{dd}, J=6.2,1.5 \mathrm{~Hz}, \mathrm{C}_{5} \mathrm{H}_{3} \mathrm{~N}\right), 6.91-6.88(58.4$ $\mathrm{H}, \mathrm{m}, \mathrm{Ph}), 6.80-6.76(29.2 \mathrm{H}, \mathrm{m}, \mathrm{Ph}), 4.13$ (18 H, s, Me). Anal. Calcd for $\mathrm{C}_{253.2} \mathrm{H}_{218} \mathrm{~B}_{7.3} \mathrm{~F}_{4.2} \mathrm{FeN}_{12} \mathrm{P}_{0.7}$ : C, 82.97; H, 6.00; N, 4.59. Found: C, 82.89; H, 6.02; N, 4.47.

Synthesis of $\left[\mathrm{Fe}^{\mathrm{II}}\left(\mathrm{Ph}_{2} \mathrm{bbpe}^{2+}\right)_{3}\right]\left[\mathrm{PF}_{6}\right]_{8}, \mathbf{1 0}$. This compound was prepared and purified in a manner similar to that for $\mathbf{8}$ by using $\left[\mathrm{Ph}_{2} \mathrm{bbpe}^{2+}\right]\left[\mathrm{PF}_{6}\right]_{2} \cdot 0.6 \mathrm{H}_{2} \mathrm{O}(153 \mathrm{mg}, 0.187 \mathrm{mmol})$ instead of $\left[\mathrm{Me}_{2} \mathrm{bbpe}^{2+}\right]\left[\mathrm{PF}_{6}\right]_{2} \cdot 0.8 \mathrm{H}_{2} \mathrm{O}$ to afford a dark blue solid: yield 121 mg, 73\%. ${ }^{1} \mathrm{H}$ NMR $\delta_{\mathrm{H}}\left(\mathrm{CD}_{3} \mathrm{CN}\right): 9.01\left(6 \mathrm{H}, \mathrm{d}, J=1.4 \mathrm{~Hz}, \mathrm{C}_{5} \mathrm{H}_{3} \mathrm{~N}\right)$, $8.92\left(12 \mathrm{H}, \mathrm{d}, J=7.1 \mathrm{~Hz}, \mathrm{C}_{5} \mathrm{H}_{4} \mathrm{~N}\right), 8.34(12 \mathrm{H}, \mathrm{d}, J=7.1 \mathrm{~Hz}$, $\left.\mathrm{C}_{5} \mathrm{H}_{4} \mathrm{~N}\right), 8.05(6 \mathrm{H}, \mathrm{d}, J=16.5 \mathrm{~Hz}, \mathrm{CH}), 7.98(6 \mathrm{H}, \mathrm{d}, J=16.5$ $\mathrm{Hz}, \mathrm{CH}), 7.77-7.71\left(36 \mathrm{H}, \mathrm{C}_{5} \mathrm{H}_{3} \mathrm{~N}+\mathrm{Ph}\right), 7.65(6 \mathrm{H}, \mathrm{d}, J=6.0$ $\mathrm{Hz}, \mathrm{C}_{5} \mathrm{H}_{3} \mathrm{~N}$ ). Anal. Calcd for $\mathrm{C}_{108} \mathrm{H}_{84} \mathrm{~F}_{48} \mathrm{FeN}_{12} \mathrm{P}_{8} \cdot 2 \mathrm{H}_{2} \mathrm{O}: \mathrm{C}$, 46.30; H, 3.17; N, 6.00. Found: C, 46.27; H, 2.91; N, 5.98.

Synthesis of $\left[\mathrm{Fe}^{\mathrm{II}}\left(\mathrm{Me}_{2} \mathbf{b b p b}^{2+}\right)_{3}\right]\left[\mathrm{PF}_{6}\right]_{8}, \mathbf{1 1}$. This compound was prepared and purified in a manner similar to that for $\mathbf{8}$ by using $\left[\mathrm{Me}_{2} \mathrm{bbpb}^{2+}\right]\left[\mathrm{PF}_{6}\right]_{2} \cdot 1.8 \mathrm{H}_{2} \mathrm{O}(131 \mathrm{mg}, 0.171 \mathrm{mmol})$ instead of $\left[\mathrm{Me}_{2} \mathrm{bbpe}^{2+}\right]\left[\mathrm{PF}_{6}\right]_{2} \cdot 0.8 \mathrm{H}_{2} \mathrm{O}$ to afford a dark blue solid: yield 85 mg, 57\%. ${ }^{1} \mathrm{H}$ NMR $\delta_{\mathrm{H}}\left(\mathrm{CD}_{3} \mathrm{CN}\right): 8.72\left(6 \mathrm{H}, \mathrm{d}, J=1.2 \mathrm{~Hz}, \mathrm{C}_{5} \mathrm{H}_{3} \mathrm{~N}\right)$, $8.48\left(12 \mathrm{H}, \mathrm{d}, J=6.8 \mathrm{~Hz}, \mathrm{C}_{5} \mathrm{H}_{4} \mathrm{~N}\right), 8.00(12 \mathrm{H}, \mathrm{d}, J=6.8 \mathrm{~Hz}$, $\left.\mathrm{C}_{5} \mathrm{H}_{4} \mathrm{~N}\right), 7.71-7.61(12 \mathrm{H}, \mathrm{CH}), 7.52(6 \mathrm{H}, \mathrm{dd}, J=6.0,1.5 \mathrm{~Hz}$, $\left.\mathrm{C}_{5} \mathrm{H}_{3} \mathrm{~N}\right), 7.43\left(6 \mathrm{H}, \mathrm{d}, J=6.1 \mathrm{~Hz}, \mathrm{C}_{5} \mathrm{H}_{3} \mathrm{~N}\right), 7.20-7.13(6 \mathrm{H}, \mathrm{m}$, $\mathrm{CH}), 7.09-7.02(6 \mathrm{H}, \mathrm{m}, \mathrm{CH}), 4.22(18 \mathrm{H}, \mathrm{s}, \mathrm{Me})$. Anal. Calcd for $\mathrm{C}_{90} \mathrm{H}_{84} \mathrm{~F}_{48} \mathrm{FeN}_{12} \mathrm{P}_{8} \cdot 4.2 \mathrm{H}_{2} \mathrm{O}: \mathrm{C}, 41.18 ; \mathrm{H}, 3.55 ; \mathrm{N}, 6.40$. Found: $\mathrm{C}$, 41.17; H, 3.24; N, 6.26.

Synthesis of $\left[\mathrm{Fe}^{\mathrm{II}}\left\{(2,4-\mathrm{DNPh})_{2} \mathrm{bbpe}^{2+}\right\}_{3}\right]\left[\mathrm{PF}_{6}\right]_{8}$, 12. This compound was prepared in a manner similar to that for $\mathbf{8}$ by using $[(2,4-$ $\left.\mathrm{DNPh})_{2} \mathrm{bbpe}^{2+}\right]\left[\mathrm{PF}_{6}\right]_{2} \cdot \mathrm{H}_{2} \mathrm{O}(175 \mathrm{mg}, 0.174 \mathrm{mmol})$ instead of $\left[\mathrm{Me}_{2} \mathrm{bbpe}^{2+}\right]\left[\mathrm{PF}_{6}\right]_{2} \cdot 0.8 \mathrm{H}_{2} \mathrm{O}$. Purification was achieved by careful reprecipitation from acetone/ethanol to afford a dark blue solid: yield $185 \mathrm{mg}, 94 \% .{ }^{1} \mathrm{H}$ NMR $\delta_{\mathrm{H}}\left(\mathrm{CD}_{3} \mathrm{CN}\right): 9.17(6 \mathrm{H}, \mathrm{d}, J=2.5$ $\left.\mathrm{Hz}, \mathrm{C}_{6} \mathrm{H}_{3}\right), 9.03\left(6 \mathrm{H}, \mathrm{d}, J=1.5 \mathrm{~Hz}, \mathrm{C}_{5} \mathrm{H}_{3} \mathrm{~N}\right), 8.85-8.82(18 \mathrm{H}$, $\left.\mathrm{C}_{6} \mathrm{H}_{3}+\mathrm{C}_{5} \mathrm{H}_{4} \mathrm{~N}\right), 8.41\left(12 \mathrm{H}, \mathrm{d}, J=7.0 \mathrm{~Hz}, \mathrm{C}_{5} \mathrm{H}_{4} \mathrm{~N}\right), 8.14-8.00$ $\left(12 \mathrm{H}, \mathrm{C}_{6} \mathrm{H}_{3}+\mathrm{CH}\right), 8.01(6 \mathrm{H}, \mathrm{d}, J=16.4 \mathrm{~Hz}, \mathrm{CH}), 7.75(6 \mathrm{H}$, $\left.\mathrm{dd}, J=5.8,1.4 \mathrm{~Hz}, \mathrm{C}_{5} \mathrm{H}_{3} \mathrm{~N}\right), 7.69\left(6 \mathrm{H}, \mathrm{d}, J=6.0 \mathrm{~Hz}, \mathrm{C}_{5} \mathrm{H}_{3} \mathrm{~N}\right)$. Anal. Calcd for $\mathrm{C}_{108} \mathrm{H}_{72} \mathrm{~F}_{48} \mathrm{FeN}_{24} \mathrm{O}_{24} \mathrm{P}_{8} \cdot 4.2 \mathrm{H}_{2} \mathrm{O}$ : C, 38.37; H, 2.40; N, 9.94. Found: C, 38.35; H, 1.97; N, 9.88.

Synthesis of $\left[\mathrm{Fe}^{\mathrm{II}}\left\{(2,4-\mathrm{DNPh})_{2} \mathrm{bbpe}^{2+}\right\}_{3}\right]\left[\mathrm{BPh}_{4}\right]_{6.55}\left[\mathrm{PF}_{6}\right]_{1.45}, 12 \mathrm{~B}$. This compound was prepared in a manner similar to that for $\mathbf{2 B}$ by using $12(31 \mathrm{mg}, 9.17 \mu \mathrm{mol})$ instead of 2 to afford a dark blue solid: yield $25 \mathrm{mg}, 61 \%$. ${ }^{1} \mathrm{H}$ NMR $\delta_{\mathrm{H}}\left(\mathrm{CD}_{3} \mathrm{CN}\right): 9.15(6 \mathrm{H}, \mathrm{d}, J=$ $\left.2.4 \mathrm{~Hz}, \mathrm{C}_{6} \mathrm{H}_{3}\right), 8.76\left(6 \mathrm{H}\right.$, dd, $\left.J=8.8,2.4 \mathrm{~Hz}, \mathrm{C}_{6} \mathrm{H}_{3}\right), 8.37-8.32$ $\left(18 \mathrm{H}, \mathrm{C}_{6} \mathrm{H}_{3}+\mathrm{C}_{5} \mathrm{H}_{4} \mathrm{~N}\right), 7.87-7.82\left(18 \mathrm{H}, \mathrm{C}_{5} \mathrm{H}_{4} \mathrm{~N}+\mathrm{C}_{5} \mathrm{H}_{3} \mathrm{~N}\right), 7.52$ $(6 \mathrm{H}, \mathrm{d}, J=16.4 \mathrm{~Hz}, \mathrm{CH}), 7.36-7.21\left(64.4 \mathrm{H}, \mathrm{C}_{6} \mathrm{H}_{3} \mathrm{~N}+\mathrm{CH}+\right.$ $\mathrm{Ph}), 7.05\left(6 \mathrm{H}, \mathrm{dd}, J=5.8,1.6 \mathrm{~Hz}, \mathrm{C}_{5} \mathrm{H}_{3} \mathrm{~N}\right), 6.94-6.86$ $(52.4 \mathrm{H}, \mathrm{Ph}), 6.81-6.74(26.2 \mathrm{H}, \mathrm{Ph})$. Anal. Calcd for $\mathrm{C}_{265.2} \mathrm{H}_{203} \mathrm{~B}_{6.55} \mathrm{~F}_{8.7} \mathrm{FeN}_{24} \mathrm{O}_{24} \mathrm{P}_{1.45}$ : C, 71.63; H, 4.60; N, 7.56. Found: C, 71.63; H, 4.58; N, 7.05. 
Table 1. Crystallographic Data and Refinement Details for the Salt $\left[\mathrm{Ph}_{2} \mathrm{bbpe}^{2+}\right]\left[\mathrm{PF}_{6}\right]_{2} \cdot 2 \mathrm{DMF}$

$\begin{array}{ll}\text { formula } & \mathrm{C}_{42} \mathrm{H}_{42} \mathrm{~F}_{12} \mathrm{~N}_{6} \mathrm{O}_{2} \mathrm{P}_{2} \\ M & 952.76 \\ \text { cryst syst } & \text { monoclinic } \\ \text { space group } & P 2_{1} / c \\ a, \AA & 9.1252(13) \\ b, \AA & 17.637(2) \\ c, \AA & 13.683(2) \\ \beta, \text { deg } & 109.478(5) \\ V, \AA^{3} & 2076.1(5) \\ Z & 2 \\ T, \mathrm{~K} & 100(2) \\ \mu, \mathrm{mm})^{-1} & 0.205 \\ \text { cryst size, mm } 3 & 0.30 \times 0.25 \times 0.20 \\ \text { cryst appearance } & \text { pale brown prism } \\ \text { no. of rflns collected } & 11919 \\ \text { no. of indep rflns }\left(R_{\text {int }}\right) & 4694(0.0315) \\ \text { no. of rflns with } I>2 \sigma(I) & 3259 \\ \text { goodness of fit on } F^{2} & 1.123 \\ \text { final R1, wR2 }(I>2 \sigma(I))^{a} & 0.0561,0.1595 \\ (\text { all data) } & 0.0802,0.1736 \\ \text { peak and hole, e } \AA^{-3} & 0.853,-0.569\end{array}$

${ }^{a}$ The structure was refined on $F_{\mathrm{o}}{ }^{2}$ using all data; the values of R1 are given for comparison with older refinements based on $F_{\mathrm{o}}$ with a typical threshold of $F_{\mathrm{o}}>4 \sigma\left(F_{\mathrm{o}}\right)$.

Synthesis of $\left[\mathrm{Fe}^{\mathrm{II}}\left(\mathrm{Me}_{2} \mathrm{bbpvb}^{2+}\right)_{3}\right]\left[\mathrm{PF}_{6}\right]_{8}, 13$. This compound was prepared and purified in a manner similar to that for $\mathbf{8}$ by using $\left[\mathrm{Me}_{2} \mathrm{bbpvb}^{2+}\right]\left[\mathrm{PF}_{6}\right]_{2} \cdot 2.8 \mathrm{H}_{2} \mathrm{O}(131 \mathrm{mg}, 0.140 \mathrm{mmol})$ instead of $\left[\mathrm{Me}_{2} \mathrm{bbpe}^{2+}\right]\left[\mathrm{PF}_{6}\right]_{2} \cdot 0.8 \mathrm{H}_{2} \mathrm{O}$. A final reprecipitation from acetonitrile/ diethyl ether afforded a dark blue solid: yield $107 \mathrm{mg}, 75 \% .{ }^{1} \mathrm{H}$ NMR $\delta_{\mathrm{H}}\left(\mathrm{CD}_{3} \mathrm{CN}\right): 8.83\left(6 \mathrm{H}, \mathrm{d}, J=1.4 \mathrm{~Hz}, \mathrm{C}_{5} \mathrm{H}_{3} \mathrm{~N}\right), 8.47(12 \mathrm{H}$, $\left.\mathrm{d}, J=6.9 \mathrm{~Hz}, \mathrm{C}_{5} \mathrm{H}_{4} \mathrm{~N}\right), 8.02\left(12 \mathrm{H}, \mathrm{d}, J=7.0 \mathrm{~Hz}, \mathrm{C}_{5} \mathrm{H}_{4} \mathrm{~N}\right)$, 7.86-7.81 $\left(36 \mathrm{H}, \mathrm{C}_{5} \mathrm{H}_{3} \mathrm{~N}+\mathrm{C}_{6} \mathrm{H}_{4}+\mathrm{CH}\right), 7.56(6 \mathrm{H}, \mathrm{dd}, J=6.1$, $\left.1.5 \mathrm{~Hz}, \mathrm{C}_{5} \mathrm{H}_{3} \mathrm{~N}\right), 7.50-7.40(18 \mathrm{H}, \mathrm{CH}), 4.21(18 \mathrm{H}, \mathrm{s}, \mathrm{Me})$. Anal. Calcd for $\mathrm{C}_{126} \mathrm{H}_{108} \mathrm{~F}_{48} \mathrm{FeN}_{12} \mathrm{P}_{8} \cdot 3.4 \mathrm{H}_{2} \mathrm{O}: \mathrm{C}, 49.34 ; \mathrm{H}, 3.77 ; \mathrm{N}, 5.48$. Found: C, 49.32; H, 3.55; N, 5.52.

X-ray Crystallography. Crystals of the proligand salt $\left[\mathrm{Ph}_{2} \mathrm{bbpe}^{2+}\right]\left[\mathrm{PF}_{6}\right]_{2} \cdot 2 \mathrm{DMF}$ were obtained by slow diffusion of diethyl ether vapor into a DMF solution. Data were collected on an Oxford Diffraction XCalibur 2 X-ray diffractometer and processed (including absorption correction) using the Oxford Diffraction CrysAlis RED software package, ${ }^{23}$ before final ins and $h k l$ files were prepared using the SHELXTL program suite. ${ }^{24}$ The structure was solved by direct methods using SIR-92 $2^{25}$ via WinGX ${ }^{26}$ and refined by full-matrix least squares on all $F_{0}{ }^{2}$ values using SHELXL-97 ${ }^{27}$ via SHELXTL. All non-hydrogen atoms were refined anisotropically, and hydrogen atoms were included in idealized positions using the riding model, with thermal parameters 1.2 times those of aryl parent carbon atoms and 1.5 times those of methyl parent carbons. The asymmetric unit contains half of one $\mathrm{Ph}_{2}$ bbpe ${ }^{2+}$ dication, one $\mathrm{PF}_{6}{ }^{-}$anion, and one DMF molecule. All other calculations were carried out using the SHELXTL package. ${ }^{24}$ Crystallographic data and refinement details are presented in Table 1.

Hyper-Rayleigh Scattering. Details of the hyper-Rayleigh scattering (HRS) experiment have been discussed elsewhere, ${ }^{28}$ and the experimental procedure used for the nanosecond measurements was as previously described. ${ }^{29}$ The latter studies were performed by using the $1064 \mathrm{~nm}$ fundamental of an injection-seeded, Q-

(23) CrysAlis RED (Version 1.171.32.4); Oxford Diffraction Ltd., Yarnton, Oxfordshire, U.K., 2006.

(24) SHELXTL (Version 6.10); Bruker AXS Inc., Madison, WI, 2000

(25) Altomare, A.; Cascarano, G.; Giacovazzo, C.; Guagliardi, A.; Burla, M. C.; Polidori, G.; Camalli, M. J. Appl. Crystallogr. 1994, 27, 435435.

(26) Farrugia, L. J. J. Appl. Crystallogr. 1999, 32, 837-838.

(27) Sheldrick, G. M. SHELXL 97, Programs for Crystal Structure Analysis (Release 97-2); University of Göttingen, Göttingen, Germany, 1997. switched Nd:YAG laser (Quanta-Ray PRO, 8 ns pulses, $7 \mathrm{~mJ}, 10$ $\mathrm{Hz}$ ). $\beta$ values were determined by using the electric-field-induced second harmonic generation $\beta_{z z z, 1064}$ for $p$-nitroaniline $\left(29 \times 10^{-30}\right.$ esu in acetonitrile) ${ }^{30}$ as an external reference. The different nature of the reference tensor component for $p$-nitroaniline has been taken into account in deriving the octupolar $\beta_{x x x}$ values for the compounds studied. The apparatus and experimental procedures used for the femtosecond HRS studies were exactly as described previously. ${ }^{31}$ These measurements were also carried out in acetonitrile, and the reference compound was crystal violet (octupolar $\beta_{x x x, 800}=327 \times$ $10^{-30}$ esu in acetonitrile; from the value of $338 \times 10^{-30} \mathrm{esu}$ in methanol, corrected for local field factors at optical frequencies). For all measurements, dilute solutions $\left(10^{-5}-10^{-6} \mathrm{M}\right)$ were used to ensure a linear dependence of $I_{2 \omega} / I_{\omega}{ }^{2}$ on concentration, precluding the need for Lambert-Beer correction factors. The absence of demodulation at $800 \mathrm{~nm}$, i.e. constant values of $\beta$ versus frequency, confirmed that no luminescence contributions to the HRS signals were present at $400 \mathrm{~nm}$, consistent with the observation of luminescence well above this wavelength (for the $\mathrm{Ru}$ compounds) or no luminescence at all (for the $\mathrm{Fe}$ compounds). The reported $\beta_{x x x, 800}$ values are the averages taken from measurements at different amplitude modulation frequencies.

Stark Spectroscopy. The Stark apparatus, experimental methods, and data collection procedure were as previously reported, ${ }^{32}$ except that a Xe arc lamp was used as the light source in the place of a $\mathrm{W}$ filament bulb. The Stark spectrum for each compound was measured at least twice. To fit the Stark data, the absorption ( $\varepsilon / v$ vs $v$ ) spectrum was modeled with a sum of Gaussian curves that reproduce the data and separate the peaks. The first and second derivatives of the Gaussian curves were then used to fit the Stark spectra with Liptay's equation. ${ }^{33}$ The dipole moment change, $\Delta \mu_{12}$ $=\mu_{\mathrm{e}}-\mu_{\mathrm{g}}$, where $\mu_{\mathrm{e}}$ and $\mu_{\mathrm{g}}$ are the respective excited- and groundstate dipole moments, associated with each of the optical transitions considered in the fit was then calculated from the coefficient of the second-derivative component. Butyronitrile was used as the glassing medium, for which the local field correction $f_{\text {int }}$ is estimated as $1.33 .^{32} \mathrm{~A}$ two-state analysis of the ICT transitions gives

$$
\Delta \mu_{\mathrm{ab}}{ }^{2}=\Delta \mu_{12}{ }^{2}+4 \mu_{12}{ }^{2}
$$

where $\Delta \mu_{\mathrm{ab}}$ is the dipole moment change between the diabatic states and $\Delta \mu_{12}$ is the observed (adiabatic) dipole moment change. The value of the transition dipole moment $\mu_{12}$ can be determined from the oscillator strength $f_{\text {os }}$ of the transition by

(28) (a) Terhune, R. W.; Maker, P. D.; Savage, C. M. Phvs. Rev. Lett 1965, 14, 681-684. (b) Clays, K.; Persoons, A. Phvs. Rev. Lett. 1991, 66, 2980-2983. (c) Clays, K.; Persoons, A. Rev. Sci. Instrum. 1992, 63, 3285-3289. (d) Hendrickx, E.; Clays, K.; Persoons, A. Acc. Chem. Res. 1998, 31, 675-683.

(29) Houbrechts, S.; Clays, K.; Persoons, A.; Pikramenou, Z.; Lehn, J.-M. Chem. Phvs. Lett. 1996, 258, 485-489.

(30) Stähelin, M.; Burland, D. M.; Rice, J. E. Chem. Phvs. Lett. 1992, 191, $245-250$.

(31) (a) Olbrechts, G.; Strobbe, R.; Clays, K.; Persoons, A. Rev. Sci. Instrum. 1998, 69, 2233-2244. (b) Olbrechts, G.; Wostyn, K.; Clays, K.; Persoons, A. Opt. Lett. 1999, 24, 403-405. (c) Clays, K.; Wostyn, K.; Olbrechts, G.; Persoons, A.; Watanabe, A.; Nogi, K.; Duan, X.M.; Okada, S.; Oikawa, H.; Nakanishi, H.; Vogel, H.; Beljonne, D.; Brédas, J.-L. J. Opt. Soc. Am. B 2000, 17, 256-265. (d) Franz, E.; Harper, E. C.; Coe, B. J.; Zahradnik, P.; Clays, K.; Asselberghs, I. Proc. SPIE-Int. Soc. Opt. Eng. 2008, 6999, 699923-1-699923-11.

(32) (a) Shin, Y. K.; Brunschwig, B. S.; Creutz, C.; Sutin, N. J. Phys. Chem. 1996, 100, 8157-8169. (b) Coe, B. J.; Harris, J. A.; Brunschwig, B. S. J. Phys. Chem. A 2002, 106, 897-905.

(33) (a) Liptay, W. In Excited States; Lim, E. C., Ed.; Academic Press: New York, 1974; Vol. 1, pp 129-229. (b) Bublitz, G. U.; Boxer, S. G. Annu. Rev. Phvs. Chem. 1997, 48, 213-242. (c) Vance, F. W.; Williams, R. D.; Hupp, J. T. Int. Rev. Phys. Chem. 1998, 17, 307329. (d) Brunschwig, B. S.; Creutz, C.; Sutin, N. Coord. Chem. Rev. 1998, 177, 61-79. 


$$
\left|\mu_{12}\right|=\left(\frac{f_{\mathrm{os}}}{1.08 \times 10^{-5} E_{\max }}\right)^{1 / 2}
$$

where $E_{\max }$ is the energy of the ICT maximum (in wavenumbers) and $\mu_{12}$ is in e $\AA$. The latter is converted into Debye units upon multiplying by 4.803 . The degree of delocalization $c_{\mathrm{b}}{ }^{2}$ and electronic coupling matrix element $H_{\mathrm{ab}}$ for the diabatic states are given by

$$
\begin{gathered}
\mathrm{c}_{\mathrm{b}}^{2}=\frac{1}{2}\left[1-\left(\frac{\Delta \mu_{12}^{2}}{\Delta \mu_{12}^{2}+4 \mu_{12}^{2}}\right)^{1 / 2}\right] \\
\left|H_{\mathrm{ab}}\right|=\left|\frac{E_{\max }\left(\mu_{12}\right)}{\Delta \mu_{\mathrm{ab}}}\right|
\end{gathered}
$$

If the hyperpolarizability $\beta_{0}$ tensor has only nonzero elements along the ICT direction, then this quantity is given by

$$
\beta_{0}=\frac{3 \Delta \mu_{12}\left(\mu_{12}\right)^{2}}{\left(E_{\max }\right)^{2}}
$$

A relative error of $\pm 20 \%$ is estimated for the $\beta_{0}$ values derived from the Stark data and using eq 5, while experimental errors of $\pm 10 \%$ are estimated for $\mu_{12}, \Delta \mu_{12}$, and $\Delta \mu_{\mathrm{ab}}, \pm 15 \%$ for $H_{\mathrm{ab}}$, and $\pm 50 \%$ for $c_{\mathrm{b}}^{2}$.

Z-Scan Measurements. All compounds were investigated as DMF solutions placed in $1 \mathrm{~mm}$ path length glass cells. The measurements were carried out at $750 \mathrm{~nm}(1.652 \mathrm{eV})$. This wavelength was chosen in such a way as to provide the optimum possible enhancement of the NLO properties of the compounds while avoiding excessive one-photon absorption. The system used for the measurements used a Clark-MXR regenerative amplifier operating at $250 \mathrm{~Hz}$ and generating ca. $800 \mu \mathrm{J}$ pulses at $775 \mathrm{~nm}$, which were used to pump a Light Conversion TOPAS traveling wave optical parametric amplifier (OPA). The wavelength for the present measurements was obtained by doubling the OPA signal.

A standard Z-scan measurement system was used, the beam from the OPA being first attenuated and spatially filtered by a pair of apertures and then focused with a lens to form a $w_{0} \approx 60 \mu \mathrm{m}$ spot at the focal plane: i.e., $z=0$. The cell was mounted on a traveling stage which was moved from $z=-40$ to $z=40 \mathrm{~mm}$. The beam transmitted through the sample was probed with a beam splitter to provide an "open aperture" signal and with an iris aperture in the far field to provide the "closed aperture" signal simultaneously. The Z-scans were analyzed using home-written software implementing the calculations as presented by Sheikh-Bahae et al. ${ }^{34}$

By comparing the shapes of computed curves with the experimental ones, we obtained the values of the nonlinear phase shift $\Delta \Phi_{0}\left(=\operatorname{Re}\left(\Delta \hat{\Phi}_{0}\right)\right)$ and the $T$ factor defined here according to the equation

$$
T=4 \pi \frac{\operatorname{Im}\left(\Delta \hat{\Phi}_{0}\right)}{\operatorname{Re}\left(\Delta \hat{\Phi}_{0}\right)}
$$

where $\Delta \hat{\Phi}_{0}$ stands for the complex phase shift. All measurements were performed in the range of intensities at which the phase shifts were below ca. $1 \mathrm{rad}$. The Z-scans were measured for three concentrations of each compound in DMF (the highest concentration being typically about $4 \mathrm{wt} \%$ ) as well as for the solvent itself, and the real and imaginary components of the $\gamma$ response of the solute were determined assuming linear dependences of the real and

(34) (a) Sheik-Bahae, M.; Said, A. A.; van Stryland, E. W. Opt. Lett. 1989 14, 955-957. (b) Sheik-Bahae, M.; Said, A. A.; Wei, T. H.; Hagan, D. J.; van Stryland, E. W. IEEE J. Ouantum Electron. 1990, 26, 760769. (c) van Stryland, E. W.; Sheik-Bahae, M. Opt. Eng. 1998, 60, 655-692. imaginary parts of the phase shift on concentration as described elsewhere. ${ }^{35}$ The data were calibrated against the nonlinearity of fused silica, which was taken to be $n_{2}=2.78 \times 10^{-16} \mathrm{~cm}^{2} \mathrm{~W}^{-1}$ at $750 \mathrm{~nm} .{ }^{35}$ The nonlinear absorption was also expressed in terms of the values of $\sigma_{2}$ calculated as shown previously. ${ }^{35}$

\section{Results and Discussion}

Synthetic Studies. The new 4,4'-disubstituted bpy derivatives bbpe, bbpb, and bbpvb were prepared in good yields via Wadsworth-Emmons reactions of 4,4'-bis-[(diethoxyphosphinyl)methyl]-2,2'-bipyridyl with the appropriate aldehyde in THF at room temperature using potassium tert-butoxide as the base (Scheme 1a). Although the related compound 4-methyl-4'-[(E)2-(4-pyridyl)vinyl]-2,2'-bipyridyl is already known, ${ }^{36}$ perhaps surprisingly, to our knowledge bbpe has not been reported previously. Dimethylation of bbpe, bbpb, and bbpvb with iodomethane in acetone proceeded smoothly to afford the salts $\left[\mathrm{Me}_{2} \mathrm{bbpe}^{2+}\right] \mathrm{I}_{2},\left[\mathrm{Me}_{2} \mathrm{bbpb}^{2+}\right] \mathrm{I}_{2}$, and $\left[\mathrm{Me}_{2} \mathrm{bbpvb}^{2+}\right] \mathrm{I}_{2}$, with no evidence for methylation of the bpy $\mathrm{N}$ atoms. The salt $[(2,4-$ $\mathrm{DNPh})_{2} \mathrm{bbpe}^{2+} \mathrm{Cl}_{2}$ was prepared in high yield via reaction of bbpe with an excess of 2,4-dinitrochlorobenzene in ethanol under reflux (Scheme 1a), as previously described for the preparation of $\left[(2,4-\mathrm{DNPh})_{2} \mathrm{qpy}^{2+}\right] \mathrm{Cl}_{2}$ (qpy $=2,2^{\prime}: 4,4^{\prime \prime}: 4^{\prime}, 4^{\prime \prime \prime}$-quaterpyridyl). ${ }^{15}$ We have previously used a Zincke-type reaction with aniline to convert (2,4-DNPh) ${ }_{2} \mathrm{qpy}^{2+}$ into $\mathrm{Ph}_{2} \mathrm{qpy}^{2+} .{ }^{37}$ However, attempts at achieving a similar reaction of $[(2,4-$ $\mathrm{DNPh})_{2} \mathrm{bbpe}^{2+} \mathrm{Cl}_{2}$ under various different conditions failed to yield any trace of $\left[\mathrm{Ph}_{2} \mathrm{bbpe}^{2+}\right] \mathrm{Cl}_{2}$. We therefore prepared the latter compound in good yield by a Knoevenagel-type reaction of $\left[\mathrm{Phpic}^{+}\right] \mathrm{Cl}$ with 2,2'-bipyridyl-4,4'-dicarboxaldehyde using acetic anhydride as the solvent and dehydration catalyst (Scheme 1b). All of the new proligand halide salts were readily converted into their corresponding hexafluorophosphate salts by anion metathesis with aqueous $\mathrm{NH}_{4} \mathrm{PF}_{6}$.

The $\mathrm{Ru}^{\mathrm{II}}$ complex salts $\mathbf{2}$ and $\mathbf{4 - 6}$ (Figure 1) were prepared via a previously reported procedure ${ }^{15}$ involving reactions of the precursor cis- $\mathrm{Ru}^{\mathrm{II}} \mathrm{Cl}_{2}(\mathrm{DMSO})_{4}{ }^{17}$ with $\left[\mathrm{Me}_{2} \mathrm{bbpe}^{2+}\right]\left[\mathrm{PF}_{6}\right]_{2}$, $\left[\mathrm{Me}_{2} \mathrm{bbpb}^{2+}\right]\left[\mathrm{PF}_{6}\right]_{2},\left[\mathrm{Me}_{2} \mathrm{bbpvb}^{2+}\right]\left[\mathrm{PF}_{6}\right]_{2}$, or $\left[\mathrm{Ph}_{2} \mathrm{bbpe}^{2+}\right]\left[\mathrm{PF}_{6}\right]_{2}$. Yields of ca. 50-65\% were obtained after column chromatographic purification. Attempts to isolate the compound $\left[\mathrm{Ru}^{\mathbb{I}}\{(2,4-\right.$ $\left.\left.\mathrm{DNPh})_{2} \mathrm{bbpe}^{2+}\right\}_{3}\right]\left[\mathrm{PF}_{6}\right]_{8}$ were unfortunately unsuccessful, probably due to decomposition of the strongly electron-deficient pyridinium units under the relatively harsh reaction conditions used (DMF under reflux). However, the use of milder conditions (DMF at room temperature) and the precursor $\mathrm{Fe}^{\mathrm{II}}\left(\mathrm{BF}_{4}\right)_{2} \cdot 6 \mathrm{H}_{2} \mathrm{O}$ allowed the synthesis of $\left[\mathrm{Fe}^{\mathrm{II}}\left\{(2,4-\mathrm{DNPh})_{2} \mathrm{bbpe}^{2+}\right\}_{3}\right]\left[\mathrm{PF}_{6}\right]_{8}(\mathbf{1 2})$ as well as the other related $\mathrm{Fe}^{\mathrm{II}}$ complex salts $\mathbf{8 , 1 0}, \mathbf{1 1}$, and $\mathbf{1 3}$ (Figure 1). The last four compounds were purified by using column chromatography and isolated in yields of ca. $55-75 \%$, while 12 was obtained pure in over $90 \%$ yield without any need for chromatographic purification.

The expected $E$ configuration of the ethenyl units in all of the proligands and complex salts is confirmed by the ${ }^{1} \mathrm{H}$ NMR coupling constants $J$ in the range ca. $15-17 \mathrm{~Hz}$ in all cases (except where signal overlap prevents these from being measured). The alternative $Z$ configuration would give $J$ values of

(35) Samoc, M.; Samoc, A.; Dalton, G. T.; Cifuentes, M. P.; Humphrey, M. G.; Fleitz, P. A. In Multiphoton Processes in Organics and Their Application; Rau, I., Kajzar, F., Eds.; Old City Publishing: Philadelphia, in press.

(36) Kim, D.; Shin, E. J. Bull. Korean Chem. Soc. 2003, 24, 1490-1494.

(37) Coe, B. J.; Harris, J. A.; Jones, L. A.; Brunschwig, B. S.; Song, K.; Clays, K.; Garín, J.; Orduna, J.; Coles, S. J.; Hursthouse, M. B. J. Am. Chem. Soc. 2005, 127, 4845-4859. 
Scheme 1. Syntheses of the New Proligand Salts $\left[\mathrm{Me}_{2} \mathrm{bbpe}^{2+}\right]\left[\mathrm{PF}_{6}\right]_{2},\left[\mathrm{Me}_{2} \mathrm{bbpb}^{2+}\right]\left[\mathrm{PF}_{6}\right]_{2},\left[\mathrm{Me}_{2} \mathrm{bbpvb}^{2+}\right]\left[\mathrm{PF}_{6}\right]_{2},\left[\left(2,4-\mathrm{DNPh}_{2} \mathrm{bbpe}^{2+}\right]\left[\mathrm{PF}_{6}\right]_{2}\right.$, and $\left[\mathrm{Ph}_{2} \mathrm{bbpe}^{2+}\right]\left[\mathrm{PF}_{6}\right]_{2}$, Together with Their Precursors bbpe, bbpb, and bbpvb ${ }^{a}$

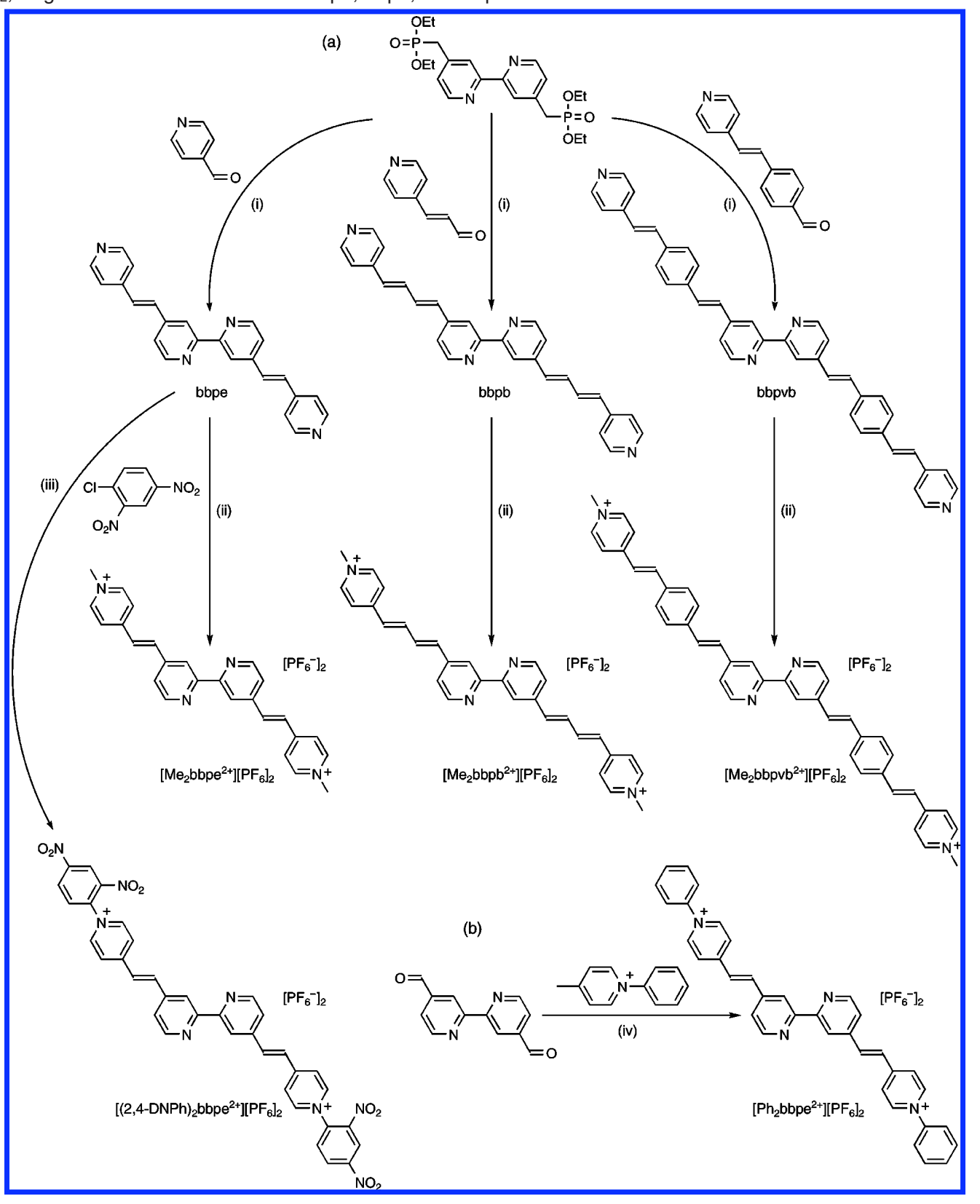

${ }^{a}$ Legend: (i) $\mathrm{KO} \mathrm{Bu}^{t} \mathrm{THF}$; (ii) $\mathrm{MeI} / \mathrm{Me}_{2} \mathrm{CO}$, then $\mathrm{NH}_{4} \mathrm{PF}_{6}$; (iii) EtOH under reflux, then $\mathrm{NH}_{4} \mathrm{PF}_{6}$; (iv) $\mathrm{Ac}_{2} \mathrm{O}$ under reflux, then $\mathrm{NH}_{4} \mathrm{PF}_{6}$.

about $11 \mathrm{~Hz}$ and also doublets shifted to relatively higher fields. We have observed no evidence for thermal isomerization/ photoisomerization of these linkages in any of the compounds studied. It is worth noting that the pyridinium-substituted "arms" of the complexes will adopt various conformations due to rotations about the $\mathrm{C}-\mathrm{C}$ single bonds. The spectroscopic and other measurements clearly probe the averaged properties of all possible structures that exist in solutions or frozen glasses. Because the $\mathrm{C}=\mathrm{C}$ bonds are held in a fixed $E$ configuration, the overall directionality of the ICT processes will not be greatly variable, but some parameters such as dipole moments may show some conformational dependency. However, given that all of the samples were studied under the same experimental conditions, comparisons between the data obtained are still expected to be valid.

For the purposes of Stark spectroscopy (see below), complex salts $2, \mathbf{8}$, and 12 were converted into their tetraphenylborate analogues (denoted $\mathbf{2 B}, \mathbf{8 B}$, and $\mathbf{1 2 B}$ ) in order to give increased solubilities in the butyronitrile glassing medium. However, both the ${ }^{1} \mathrm{H}$ NMR spectra and $\mathrm{CHN}$ analyses show that anion metathesis is not complete, with as much as ca. 1.5 equiv of $\mathrm{PF}_{6}{ }^{-}$being retained in the products; this observation is unsurprising given the unusually large number of anions associated with each complex cation.

Electronic Spectroscopy Studies. The electronic absorption spectra of the new complex salts 2, 4-6, 8, and 10-13 have been measured in acetonitrile, and the data are presented in Table 2 . These spectra feature intense intraligand $\pi \rightarrow \pi^{*}$ absorptions in the UV region, together with intense, broad $\mathrm{d}\left(\mathrm{M}^{\mathrm{II}}\right) \rightarrow \pi^{*}\left(\mathrm{~L}^{\mathrm{A}}\right)$ $\left(\mathrm{M}=\mathrm{Ru} / \mathrm{Fe}, \mathrm{L}^{\mathrm{A}}=\mathrm{N}\right.$-substituted bpy derivative) visible MLCT bands, with two maxima in each case. The energy separation between the two MLCT maxima lies in the range of $0.45-0.48$ $\mathrm{eV}$ for the $\mathrm{Ru}^{\mathrm{II}}$ complexes and $0.48-0.58 \mathrm{eV}$ for their $\mathrm{Fe}^{\mathrm{II}}$ analogues. The data for only the MLCT bands are collected in 


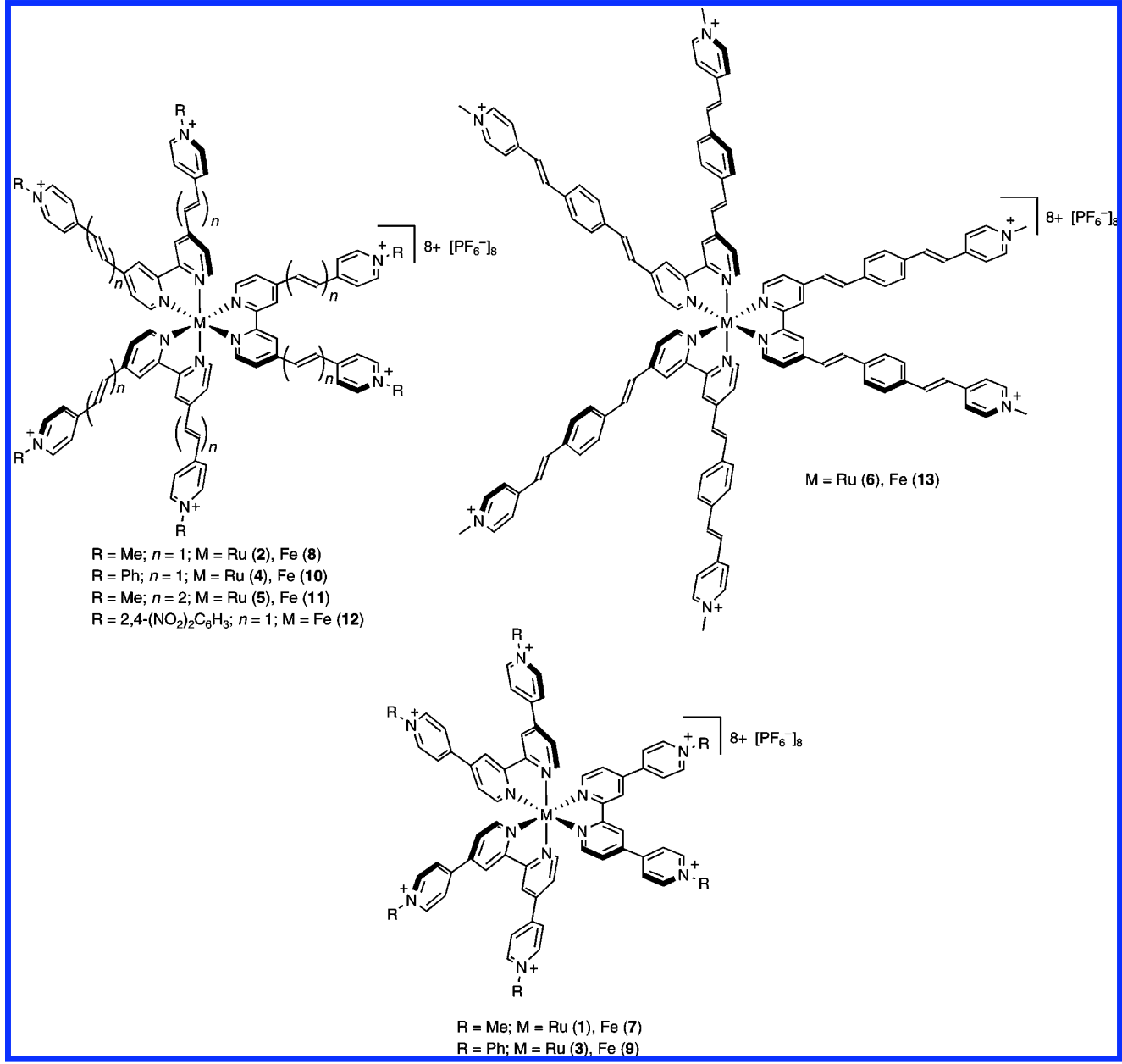

Figure 1. Chemical structures of the new complex salts investigated, together with the previously reported $\mathbf{1}, \mathbf{3}, \mathbf{7}$, and $\mathbf{9} .{ }^{15}$

Table 3, together with those for the previously reported related compounds $\mathbf{1}, \mathbf{3}, \mathbf{7}$, and $\mathbf{9}^{15}$ for comparison purposes. Representative UV-vis spectra of the complex salts $\mathbf{1}, \mathbf{2}, \mathbf{4 - 6}, \mathbf{1 0}$, and $\mathbf{1 3}$ are shown in Figures 2 and 3.

Considering the MLCT absorptions of the $\mathrm{Ru}^{\mathrm{II}}$ complexes $\mathbf{1}$, $\mathbf{2}$, and $\mathbf{5}$ (Figure 2), the lower energy (LE) band shows a steady red shift as the conjugation within the ligands is extended, with sequential energy differences of 0.10 and $0.03 \mathrm{eV}$ (Table 3). The energy of the higher energy (HE) band decreases by 0.25 $\mathrm{eV}$ on moving from $\mathbf{1}$ to $\mathbf{2}$ but then remains constant in $\mathbf{5}$, although it should be noted that the error limits on the quoted HE maxima are large due to strong overlap with the LE band. The band intensities also increase steadily and substantially on moving along this series, due to the increasing extent of $\pi$-coupling. Comparison of the data for the $N$-Ph derivatives 3 and 4 reveals a similar pattern, with red shifts of 0.09 and 0.16 $\mathrm{eV}$ for the LE and HE bands, respectively. A concomitant ca. 2-fold enhancement in $\epsilon$ is also observed. The $\mathrm{Me}_{2}$ bbpvb ${ }^{2+}$ containing complex in $\mathbf{6}$ shows only one discernible MLCT maximum (but with a high-energy shoulder; Figure 2) that lies to high energy of those of all the other new $\mathrm{Ru}^{\mathrm{II}}$ chromophores, but to slightly lower energy in comparison with the qpy-based species $\mathbf{1}$ and 3. A major difference between the latter and the new complexes is the increased intensities of the ILCT bands; for $\mathbf{1}$ and 3, the lowest energy such absorptions are observed at $324 \mathrm{~nm}\left(\epsilon=55.0 \times 10^{3} \mathrm{M}^{-1} \mathrm{~cm}^{-1}\right)$ and $328 \mathrm{~nm}(\epsilon=65.7 \times$ $\left.10^{3} \mathrm{M}^{-1} \mathrm{~cm}^{-1}\right)$, respectively. ${ }^{15}$ As for the MLCT bands, this change is attributable to the presence of extended and more strongly coupled $\pi$-conjugated systems in $\mathbf{2}$ and $\mathbf{4 - 6}$. The MLCT $\epsilon$ values quoted for $\mathbf{6}$ and $\mathbf{1 3}$ are strongly enhanced by overlap with the ILCT bands (Figures 2 and 3).

As noted previously for the qpy-based chromophores, ${ }^{15}$ the MLCT bands of the $\mathrm{Fe}^{\mathrm{II}}$ complexes are somewhat less intense and red-shifted in comparison with those of their $\mathrm{Ru}^{\mathrm{II}}$ counterparts (Figure 3). On moving from 1 to 7 , the energy decrease for the LE band is ca. $0.39 \mathrm{eV}$ and that for the $\mathrm{HE}$ band is ca. $0.33 \mathrm{eV}$. The corresponding changes for the pair $\mathbf{3}$ and $\mathbf{9}$ are 0.38 and $0.29 \mathrm{eV}$. For the new complexes, the shift of the LE absorption $(-0.35 \mathrm{eV}$ in all cases) on replacing $\mathrm{Ru}$ with $\mathrm{Fe}$ is slightly smaller than those for the analogous qpy-based chromophores. The shift for the HE band appears to vary over a range of ca. $0.25-0.30 \mathrm{eV}$, but this may not really be the case, due to the uncertainties in the $E_{\max }$ values. In contrast to the behavior of the MLCT absorptions, the intraligand bands are more intense in the $\mathrm{Fe}^{\mathrm{II}}$ complexes in comparison with their $\mathrm{Ru}^{\mathrm{II}}$ analogues. In keeping with our previous studies on various chromophores with $N$-arylpyridinium electron acceptor groups, ${ }^{15,20,37}$ replacing a methyl with a phenyl substituent causes the MLCT bands to red-shift in every case; these decreases in energy for the HE bands are generally about twice those of the LE absorptions (Table 3). On moving from 10 to its $(2,4-\mathrm{DNPh})_{2} \mathrm{bbpe}^{2+}$ counterpart in 12, further small red shifts are observed.

Taken in the context of the vast literature on transition-metal tris-chelate complexes of $\alpha$-diimine ligands, our new chromophores, and especially the $\mathrm{Me}_{2} \mathrm{bbpvb}^{2+}$-containing species 
Table 2. UV-Vis Absorption and Electrochemical Data for the New Complex Salts $\left[\mathrm{M}^{\prime \prime}\left(\mathrm{L}^{\mathrm{A}}\right)_{3}\right]\left[\mathrm{PF}_{6}\right]_{8}$ in Acetonitrile

\begin{tabular}{|c|c|c|c|c|c|}
\hline \multirow[b]{2}{*}{ salt $\left(M / L^{A}\right)$} & \multirow[b]{2}{*}{$\lambda_{\max }, \mathrm{nm}^{a}\left(\epsilon, 10^{3} \mathrm{M}^{-1} \mathrm{~cm}^{-1}\right)$} & \multirow[b]{2}{*}{$E_{\max }, \mathrm{eV}$} & \multirow[b]{2}{*}{ assignment } & \multicolumn{2}{|c|}{$E_{1 / 2}, \mathrm{~V}$ vs $\mathrm{Ag} / \mathrm{AgCl}\left(\Delta E_{\mathrm{p}}, \mathrm{mV}\right)^{b}$} \\
\hline & & & & $M^{\|\| / I I}$ & $\mathrm{~L}^{\mathrm{A}}$ reduction \\
\hline \multirow[t]{3}{*}{$2\left(\mathrm{Ru} / \mathrm{Me}_{2} \mathrm{bbpe}^{2+}\right)$} & $506(58.9)$ & 2.45 & $\mathrm{~d} \rightarrow \pi^{*}$ & $1.43(90)$ & $-0.67(190)$ \\
\hline & $428(40.5)$ & 2.90 & $\mathrm{~d} \rightarrow \pi^{*}$ & & $-0.99^{c}$ \\
\hline & 314 (159.4) & 3.95 & $\pi \rightarrow \pi^{*}$ & & \\
\hline \multirow[t]{3}{*}{$4\left(\mathrm{Ru} / \mathrm{Ph}_{2} \mathrm{bbpe}^{2+}\right)$} & $514(75.6)$ & 2.41 & $\mathrm{~d} \rightarrow \pi^{*}$ & $1.42(80)$ & $-0.52^{c}$ \\
\hline & 434 (49.2) & 2.86 & $\mathrm{~d} \rightarrow \pi^{*}$ & & $-0.94(130)$ \\
\hline & $329(206.5)$ & 3.77 & $\pi \rightarrow \pi^{*}$ & & \\
\hline \multirow[t]{4}{*}{$5\left(\mathrm{Ru} / \mathrm{Me}_{2} \mathrm{bbpb}^{2+}\right)$} & $513(80.1)$ & 2.42 & $\mathrm{~d} \rightarrow \pi^{*}$ & $1.32(80)$ & $-0.67^{c}$ \\
\hline & $428(61.6)$ & 2.90 & $\mathrm{~d} \rightarrow \pi^{*}$ & & $-1.13^{c}$ \\
\hline & $351(215.4)$ & 3.53 & $\pi \rightarrow \pi^{*}$ & & \\
\hline & $255(61.1)$ & 4.86 & $\pi \rightarrow \pi^{*}$ & & \\
\hline \multirow{4}{*}{$6\left(\mathrm{Ru} / \mathrm{Me}_{2} \mathrm{bbpvb}^{2+}\right)$} & $501(106.1)$ & 2.48 & $\mathrm{~d} \rightarrow \pi^{*}$ & $1.23(90)$ & $-0.83^{c}$ \\
\hline & $385(275.0)$ & 3.22 & $\pi \rightarrow \pi^{*}$ & & $-0.99^{c}$ \\
\hline & 307 (61.5) & 4.04 & $\pi \rightarrow \pi^{*}$ & & $-1.78^{c}$ \\
\hline & $247(88.2)$ & 5.02 & $\pi \rightarrow \pi^{*}$ & & \\
\hline \multirow[t]{4}{*}{$8\left(\mathrm{Fe} / \mathrm{Me}_{2} \mathrm{bbpe}^{2+}\right)$} & $591(40.9)$ & 2.10 & $\mathrm{~d} \rightarrow \pi^{*}$ & $1.19(80)$ & $-0.67(180)$ \\
\hline & 468 (26.9) & 2.65 & $\mathrm{~d} \rightarrow \pi^{*}$ & & $-1.10^{c}$ \\
\hline & $317(190.0)$ & 3.91 & $\pi \rightarrow \pi^{*}$ & & \\
\hline & $221(60.2)$ & 2.21 & $\pi \rightarrow \pi^{*}$ & & \\
\hline \multirow[t]{3}{*}{$10\left(\mathrm{Fe} / \mathrm{Ph}_{2} \mathrm{bbpe}^{2+}\right)$} & $601(50.7)$ & 2.06 & $\mathrm{~d} \rightarrow \pi^{*}$ & $1.19(90)$ & $-0.54^{c}$ \\
\hline & 485 (31.7) & 2.56 & $\mathrm{~d} \rightarrow \pi^{*}$ & & $-0.96(120)$ \\
\hline & 336 (241.9) & 3.69 & $\pi \rightarrow \pi^{*}$ & & \\
\hline \multirow[t]{4}{*}{$11\left(\mathrm{Fe} / \mathrm{Me}_{2} \mathrm{bbpb}^{2+}\right)$} & $600(56.6)$ & 2.07 & $\mathrm{~d} \rightarrow \pi^{*}$ & $1.08(90)$ & $-0.71^{c}$ \\
\hline & 475 (38.5) & 2.61 & $\mathrm{~d} \rightarrow \pi^{*}$ & & $-1.18^{c}$ \\
\hline & 358 (274.7) & 3.46 & $\pi \rightarrow \pi^{*}$ & & \\
\hline & $251(61.9)$ & 4.94 & $\pi \rightarrow \pi^{*}$ & & \\
\hline \multirow[t]{5}{*}{$12\left(\mathrm{Fe} /(2,4-\mathrm{DNPh})_{2} \mathrm{bbpe}^{2+}\right)$} & $611(50.0)$ & 2.03 & $\mathrm{~d} \rightarrow \pi^{*}$ & $1.21(100)$ & $-0.30^{c}$ \\
\hline & 495 (29.2) & 2.51 & $\mathrm{~d} \rightarrow \pi^{*}$ & & $-0.98^{c}$ \\
\hline & $333(228.1)$ & 3.72 & $\pi \rightarrow \pi^{*}$ & & $-1.31^{c}$ \\
\hline & $226(159.0)$ & 5.49 & $\pi \rightarrow \pi^{*}$ & & $-1.39^{c}$ \\
\hline & & & & & $-1.53^{c}$ \\
\hline \multirow{4}{*}{$13\left(\mathrm{Fe} / \mathrm{Me}_{2} \mathrm{bbpvb}^{2+}\right)$} & $581(61.5)$ & 2.13 & $\mathrm{~d} \rightarrow \pi^{*}$ & $0.97(80)$ & $-0.87^{c}$ \\
\hline & 458 (67.9) & 2.71 & $\mathrm{~d} \rightarrow \pi^{*}$ & & $-1.05^{c}$ \\
\hline & 385 (349.8) & 3.22 & $\pi \rightarrow \pi^{*}$ & & $-1.84^{c}$ \\
\hline & $246(93.8)$ & 5.04 & $\pi \rightarrow \pi^{*}$ & & \\
\hline
\end{tabular}

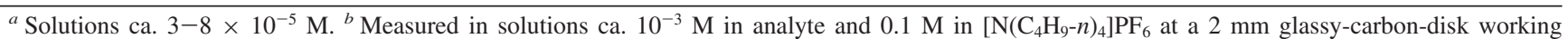
electrode with a scan rate of $200 \mathrm{mV} \mathrm{s}^{-1}$. Ferrocene was used as the internal reference: $E_{1 / 2}=0.43 \mathrm{~V}, \Delta E_{\mathrm{p}}=80-90 \mathrm{mV}$. ${ }^{c} E_{\mathrm{pc}}$ for an irreversible reduction process.

6 and 13, display unusually intense ICT absorptions. The underivatized complexes $\left[\mathrm{M}^{\mathrm{II}}(\mathrm{bpy})_{3}\right]^{2+}$ give MLCT $\epsilon$ values of ca. $1.3 \times 10^{4} \mathrm{M}^{-1} \mathrm{~cm}^{-1}$ for $\mathrm{M}=\mathrm{Ru}\left(\lambda_{\max } 452 \mathrm{~nm}\right.$ in acetonitrile $)^{38}$ and ca. $0.9 \times 10^{4} \mathrm{M}^{-1} \mathrm{~cm}^{-1}$ for $\mathrm{M}=\mathrm{Fe}\left(\lambda_{\max }\right.$ $522 \mathrm{~nm}$ in water). ${ }^{39}$ In comparison with the latter, the LE MLCT band of $\mathbf{1 3}$ is approximately 7 times more intense (Table 2). Comparisons with more closely related species substituted with extended $\pi$-conjugated systems are of more immediate interest. Le Bozec and colleagues have reported a number of such species, and the most intense absorption band apparently reported is for a $\mathrm{Zn}^{\mathrm{II}}$ complex of an azobenzene-derivatized bpy ligand. ${ }^{1 \mathrm{e}}$ Thus, replacing the outer vinyl units in $\mathrm{Me}_{2} \mathrm{bbpvb}{ }^{2+}$ with $\mathrm{N}=\mathrm{N}$ and swapping the $N$-methylpyridinium groups for $-\mathrm{N}\left({ }^{\mathrm{n}} \mathrm{Bu}\right)_{2}$ gives an $\epsilon$ value of $2.46 \times 10^{5} \mathrm{M}^{-1} \mathrm{~cm}^{-1}$ for the ILCT band ( $\lambda_{\max } 496 \mathrm{~nm}$ in dichloromethane); this intensity is slightly smaller than that observed for $\mathbf{6}$ but only about $70 \%$ of that for 13. Indeed, to our knowledge the ILCT band for $\mathbf{1 3}$ is the most intense single absorption feature yet reported for a mononuclear complex of this type.

Electrochemical Studies. The new complex salts 2, 4-6, 8, and 10-13 have been studied by cyclic voltammetry in acetonitrile, and the results are presented in Table 2. Selected data are also collected together with those for the previously

(38) Juris, A.; Balzani, V.; Belser, P.; von Zelewsky, A. Helv. Chim. Acta 1981, 64, 2175-2182.

(39) Ford-Smith, M. H.; Sutin, N. J. Am. Chem. Soc. 1961, 83, 18301834. reported qpy-based species $\mathbf{1}, \mathbf{3}, \mathbf{7}$, and $\mathbf{9}^{15}$ in Table 3. In general, these compounds give better defined electrochemistry when using a glassy-carbon working electrode rather than a platinum disk $;{ }^{15}$ therefore, only data obtained by using the former are quoted here. Representative cyclic voltammograms of the complex salts $\mathbf{4}$ and $\mathbf{1 0}$ are shown in Figure 4.

All of the complexes show reversible or quasi-reversible $\mathrm{M}^{\mathrm{II} / \mathrm{II}}(\mathrm{M}=\mathrm{Ru}, \mathrm{Fe})$ oxidation waves, together with reversible, quasi-reversible, and/or irreversible ligand-based reductions. The most obvious trend evident in these data is that the $E_{1 / 2}\left(\mathrm{Fe}^{\mathrm{III} / \mathrm{II}}\right)$ values are lower than the corresponding potentials for their $\mathrm{Ru}$ analogues by $210-260 \mathrm{mV}$ (Figure 4), a consequence of the higher electron density at the Fe ${ }^{I I}$ centers (Table 3$) .{ }^{15}$ This difference is also reflected in the red shifts observed for the MLCT bands (see above). For a given conjugated system in $\mathrm{L}^{\mathrm{A}}$, the $E_{1 / 2}\left(\mathrm{M}^{\mathrm{III} / \mathrm{II}}\right)$ values are not significantly affected by the nature of the $\mathrm{N}$ substituent, as observed for example in the series 8, 10, and 12. However, extending the conjugation within the ligands does affect the potentials. For the series 1, 2, 5, and $\mathbf{6}$, $E_{1 / 2}\left(\mathrm{Ru}^{\mathrm{III} / \mathrm{II}}\right)$ decreases by respective increments of 170,110 , and $90 \mathrm{mV}$, while for the series 7, 8, 11, and $\mathbf{1 3}$ the sequential changes in $E_{1 / 2}\left(\mathrm{Fe}^{\mathrm{III} / \mathrm{II}}\right)$ are 200,110 , and $110 \mathrm{mV}$. This trend is attributable to the cumulative electron-donating influence of the ethylene substituents, and it is apparent that the $(E, E)-1,4-$ bis(vinyl)phenylene unit is more strongly donating than the $(E, E)$-1,3-butadienyl fragment. By way of comparison, inserting an $(E)$-vinyl unit into the $N$-methyl-4,4'-bipyridinium ligand in 
Table 3. MLCT Absorption and Selected Electrochemical Data for Complex Salts 1-13 in Acetonitrile

\begin{tabular}{|c|c|c|c|c|}
\hline \multirow[b]{2}{*}{ salt $\left(M / L^{A}\right)$} & \multirow[b]{2}{*}{$\lambda_{\max }, \mathrm{nm}^{a}\left(\epsilon, 10^{3} \mathrm{M}^{-1} \mathrm{~cm}^{-1}\right)$} & \multirow[b]{2}{*}{$E_{\max }, \mathrm{eV}$} & \multicolumn{2}{|c|}{$E_{1 / 2}$ or $E, \mathrm{~V}$ vs $\mathrm{Ag} / \mathrm{AgCl}^{b}$} \\
\hline & & & $\mathrm{M}^{\|1 /\| I}$ & $\mathrm{~L}^{\mathrm{A}}$ reduction \\
\hline \multirow{2}{*}{$1\left(\mathrm{Ru} / \mathrm{Me}_{2} \mathrm{qpy} y^{2+}\right)^{c}$} & $486(37.3)$ & 2.55 & 1.60 & -0.70 \\
\hline & $394(26.4)$ & 3.15 & & \\
\hline $2\left(\mathrm{Ru} / \mathrm{Me}_{2} \mathrm{bbpe}^{2+}\right)$ & $428(40.5)$ & 2.90 & & \\
\hline \multirow[t]{2}{*}{$3\left(\mathrm{Ru} / \mathrm{Ph}_{2} \mathrm{qpy}^{2+}\right)^{c}$} & $496(37.3)$ & 2.50 & 1.61 & -0.53 \\
\hline & $410(26.1)$ & 3.02 & & \\
\hline \multirow{2}{*}{$5\left(\mathrm{Ru} / \mathrm{Me}_{2} \mathrm{bbpb}^{2+}\right)$} & $513(80.1)$ & 2.42 & 1.32 & $-0.67^{d}$ \\
\hline & 428 (61.6) & 2.90 & & \\
\hline $6\left(\mathrm{Ru} / \mathrm{Me}_{2} \mathrm{bbpvb}^{2+}\right)$ & $501(106.1)$ & 2.48 & 1.23 & $-0.83^{d}$ \\
\hline \multirow[t]{2}{*}{$7\left(\mathrm{Fe} / \mathrm{Me}_{2} \mathrm{qpy}^{2+}\right)^{c}$} & $574(26.0)$ & 2.16 & 1.39 & $-0.72^{d}$ \\
\hline & $440(18.8)$ & 2.82 & & \\
\hline \multirow[t]{2}{*}{$8\left(\mathrm{Fe} / \mathrm{Me}_{2} \mathrm{bbpe}^{2+}\right)$} & $591(40.9)$ & 2.10 & 1.19 & -0.67 \\
\hline & $468(26.9)$ & 2.65 & & \\
\hline \multirow[t]{2}{*}{$11\left(\mathrm{Fe} / \mathrm{Me}_{2} \mathrm{bbpb}^{2+}\right)$} & $600(56.6)$ & 2.07 & 1.08 & $-0.71^{d}$ \\
\hline & $475(38.5)$ & 2.61 & & \\
\hline \multirow[t]{2}{*}{$12\left(\mathrm{Fe} /(2,4-\mathrm{DNPh})_{2} \mathrm{bbpe}^{2+}\right)$} & $611(50.0)$ & 2.03 & 1.21 & $-0.30^{d}$ \\
\hline & $495(29.2)$ & 2.51 & & \\
\hline \multirow[t]{2}{*}{$13\left(\mathrm{Fe} / \mathrm{Me}_{2} \mathrm{bbpvb}^{2+}\right)$} & $581(61.5)$ & 2.13 & 0.97 & $-0.87^{d}$ \\
\hline & $458(67.9)$ & 2.71 & & \\
\hline
\end{tabular}

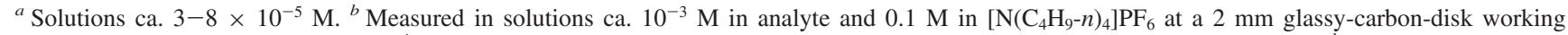
electrode with a scan rate of $200 \mathrm{mV} \mathrm{s}{ }^{-1}$. Ferrocene was used as the internal reference: $E_{1 / 2}=0.43 \mathrm{~V}$. ${ }^{c}$ Data taken from ref $15 .{ }^{d} E_{\mathrm{pc}}$ for an irreversible reduction process.

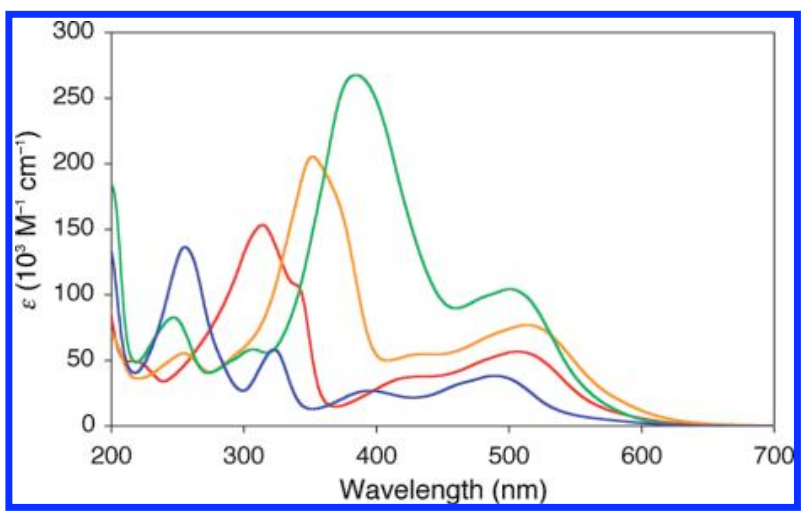

Figure 2. Electronic absorption spectra of 1 (blue), 2 (red), 5 (gold), and 6 (green) at $293 \mathrm{~K}$ in acetonitrile.

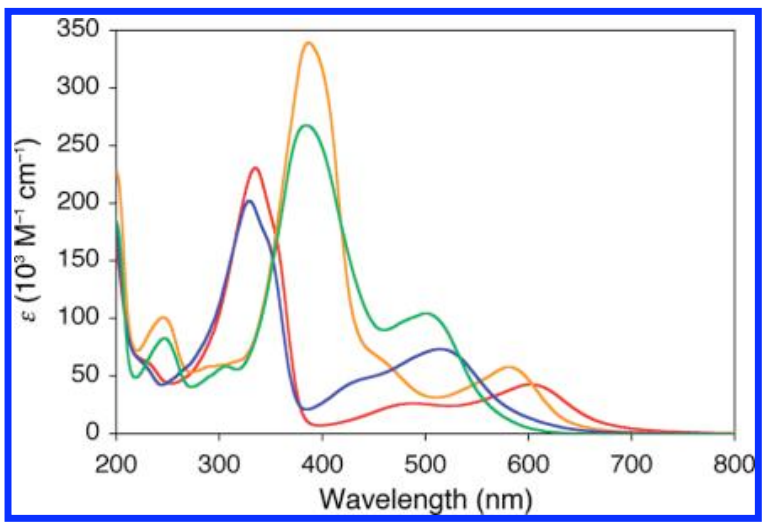

Figure 3. Electronic absorption spectra of 4 (blue), 6 (green), 10 (red), and $\mathbf{1 3}$ (gold) at $293 \mathrm{~K}$ in acetonitrile.

1D dipolar complexes causes $E_{1 / 2}\left(\mathrm{M}^{\mathrm{II} / \mathrm{II}}\right)$ to decrease by ca. $30-50 \mathrm{mV}$, while moving to a $(E, E)$-1,3-butadienyl or a $(E, E)$ -

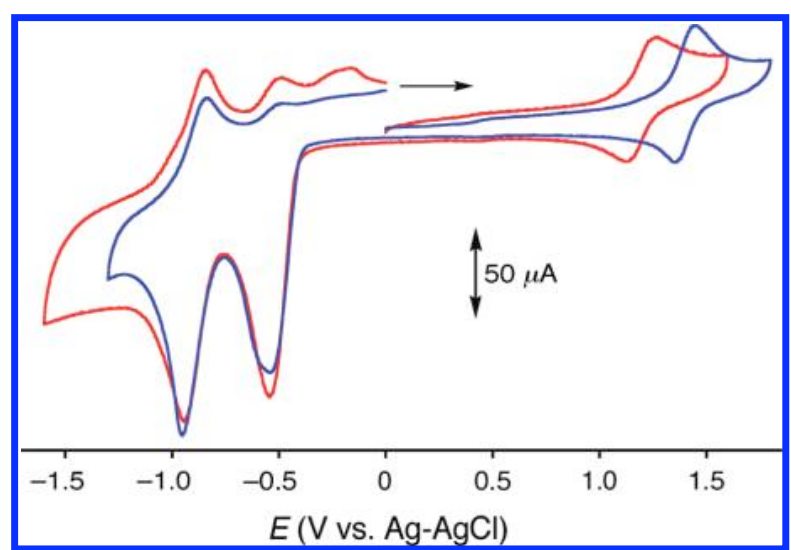

Figure 4. Cyclic voltammograms of $\mathbf{4}$ (blue) and $\mathbf{1 0}$ (red) in acetonitrile at $200 \mathrm{mV} \mathrm{s}^{-1}$ at a glassy-carbon working electrode (the arrow indicates the direction of the initial scans).

1,4-bis(vinyl)phenylene bridge gives smaller additional changes of $0-30 \mathrm{mV} \cdot{ }^{21,40}$ These decreases in the $E_{1 / 2}\left(\mathrm{M}^{\mathrm{III} / \mathrm{II}}\right)$ values correlate with the steady red shifts observed in the LE MLCT bands (see above), except for the $\mathrm{Me}_{2} \mathrm{bbpvb}^{2+}$ compounds.

The often irreversible nature of the ligand-based reduction processes precludes further extensive discussion. However, it is apparent that the nature of the metal center does not significantly affect the potentials. Also, the first reduction wave is markedly sensitive to the $\mathrm{N}$ substituent, with all of the $\mathrm{N}$-phenyl complexes showing higher potentials in comparison with their $N$-methyl analogues. A total anodic shift of $370 \mathrm{mV}$ is observed moving along the series $\mathbf{8 , 1 0}$, and 12. This trend reflects the increasing electron-accepting ability of the pyri-

(40) Coe, B. J.; Foxon, S. P.; Harper, E. C.; Raftery, J.; Shaw, R.; Swanson, C. A.; Asselberghs, I.; Clays, K.; Brunschwig, B. S.; Fitch, A. G. Inorg. Chem. 2009, 48, 1370-1379. 


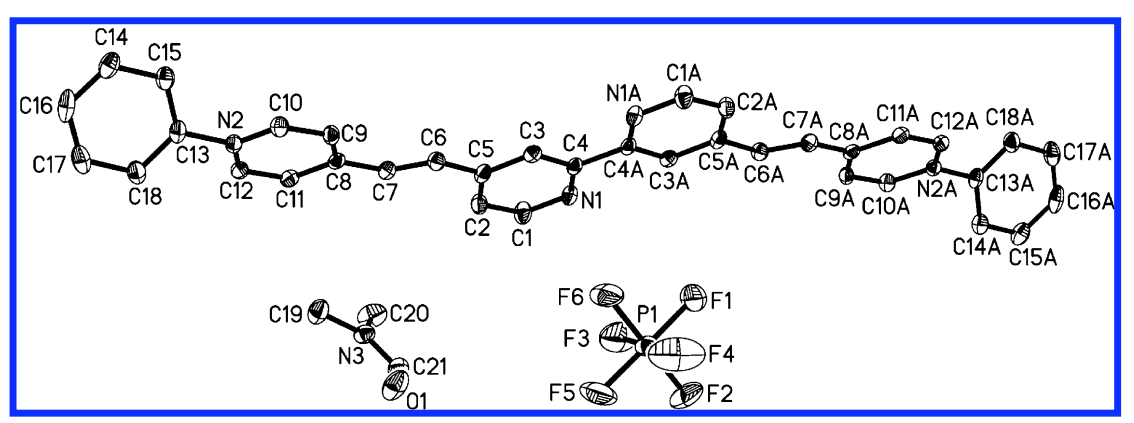

Figure 5. Representation of the molecular structure of the salt $\left[\mathrm{Ph}_{2} \mathrm{bbpe}^{2+}\right]\left[\mathrm{PF}_{6}\right]_{2} \cdot 2 \mathrm{DMF}$, with $\mathrm{H}$ atoms omitted for clarity (50\% probability ellipsoids). While the $\mathrm{Ph}_{2}$ bbpe $^{2+}$ cation has been completed by generating symmetry-equivalent atoms, only the single $\mathrm{PF}_{6}{ }^{-}$anion and $\mathrm{DMF}$ molecule present in the asymmetric unit are shown.

Table 4. Selected Interatomic Distances $(\AA ̊)$ and Angles (deg) for the Salt $\left[\mathrm{Ph}_{2} \mathrm{bbpe}^{2+}\right]\left[\mathrm{PF}_{6}\right]_{2} \cdot 2 \mathrm{DMF}$

\begin{tabular}{llll}
\hline $\mathrm{N}(1)-\mathrm{C}(4)$ & $1.336(3)$ & $\mathrm{C}(8)-\mathrm{C}(9)$ & $1.392(3)$ \\
$\mathrm{N}(1)-\mathrm{C}(1)$ & $1.333(3)$ & $\mathrm{C}(8)-\mathrm{C}(11)$ & $1.394(3)$ \\
$\mathrm{C}(3)-\mathrm{C}(4)$ & $1.380(3)$ & $\mathrm{C}(9)-\mathrm{C}(10)$ & $1.356(3)$ \\
$\mathrm{C}(1)-\mathrm{C}(2)$ & $1.373(3)$ & $\mathrm{C}(11)-\mathrm{C}(12)$ & $1.349(3)$ \\
$\mathrm{C}(3)-\mathrm{C}(5)$ & $1.391(3)$ & $\mathrm{N}(2)-\mathrm{C}(10)$ & $1.358(3)$ \\
$\mathrm{C}(2)-\mathrm{C}(5)$ & $1.385(3)$ & $\mathrm{N}(2)-\mathrm{C}(12)$ & $1.354(3)$ \\
$\mathrm{C}(5)-\mathrm{C}(6)$ & $1.454(3)$ & $\mathrm{N}(2)-\mathrm{C}(13)$ & $1.436(3)$ \\
$\mathrm{C}(6)-\mathrm{C}(7)$ & $1.325(3)$ & $\mathrm{C}(4)-\mathrm{C}(4) \mathrm{A}$ & $1.485(4)$ \\
$\mathrm{C}(7)-\mathrm{C}(8)$ & $1.449(3)$ & & \\
\hline $\mathrm{C}(5)-\mathrm{C}(6)-\mathrm{C}(7)$ & $123.1(2)$ & $\mathrm{C}(6)-\mathrm{C}(7)-\mathrm{C}(8)$ & $127.4(2)$ \\
\hline
\end{tabular}

dinium groups, as also manifested in the MLCT data (see above) and in previously reported related compounds. ${ }^{15,20,37}$ The relatively large currents for the cathodic peaks in comparison with those for the metal-based waves (Figure 4) are consistent with multielectron processes attributable to simultaneous reductions of all three $\mathrm{L}^{\mathrm{A}}$ ligands.

Crystallographic Study. Although we have unfortunately been unable to grow diffraction-quality crystals of any of the new complex salts, a single-crystal X-ray structure has been obtained for the proligand salt $\left[\mathrm{Ph}_{2} \mathrm{bbpe}^{2+}\right]\left[\mathrm{PF}_{6}\right]_{2} \cdot 2 \mathrm{DMF}$. A representation of the molecular structure is shown in Figure 5, and selected interatomic distances and angles are presented in Table 4. We have also determined structures of the related compounds $\left[\mathrm{Ph}_{2} \mathrm{qpy}^{2+}\right]\left[\mathrm{PF}_{6}\right]_{2} \cdot \mathrm{Me}_{2} \mathrm{CO}^{37}$ and $\left[\mathrm{Phbpe}^{+}\right] \mathrm{Cl} \cdot \mathrm{HCl} \cdot \mathrm{H}_{2} \mathrm{O} .{ }^{41}$

As expected on the basis of steric considerations, the $\mathrm{Ph}_{2}$ bbpe ${ }^{2+}$ cation adopts a transoid configuration with a crystallographic center of symmetry in the middle of the $\mathrm{C}-\mathrm{C}$ bond between the rings of the bpy group. The same applies to the related cation in $\left[\mathrm{Ph}_{2} \mathrm{qpy}^{2+}\right]\left[\mathrm{PF}_{6}\right]_{2} \cdot \mathrm{Me}_{2} \mathrm{CO}{ }^{37}$ The $\pi$-conjugated bbpe unit in $\left[\mathrm{Ph}_{2} \mathrm{bbpe}^{2+}\right]\left[\mathrm{PF}_{6}\right]_{2} \cdot 2 \mathrm{DMF}$ is close to planar, with a dihedral angle of $7.9^{\circ}$ between the two pyridyl rings; this compares with a corresponding angle of $15.7^{\circ}$ in $\left[\mathrm{Phbpe}^{+}\right] \mathrm{Cl} \cdot \mathrm{HCl} \cdot \mathrm{H}_{2} \mathrm{O} .{ }^{41}$ The dihedral angle between the quaternized pyridyl and attached phenyl rings in the latter is $53.4^{\circ}, 41$ somewhat larger than that observed in $\left[\mathrm{Ph}_{2} \mathrm{bbpe}^{2+}\right]\left[\mathrm{PF}_{6}\right]_{2} \cdot 2 \mathrm{DMF}$ $\left(44.5^{\circ}\right)$. Such twists are attributable in part to steric interactions between the ortho hydrogen atoms of the two rings. All of the other geometric parameters are closely similar for both $\left[\mathrm{Ph}_{2} \mathrm{bbpe}^{2+}\right]\left[\mathrm{PF}_{6}\right]_{2} \cdot 2 \mathrm{DMF}$ and $\left[\mathrm{Phbpe}^{+}\right] \mathrm{Cl} \cdot \mathrm{HCl} \cdot \mathrm{H}_{2} \mathrm{O}{ }^{41}$

Hyper-Rayleigh Scattering and Luminescence Studies. The $\beta$ values of the complex salts $\mathbf{2}, \mathbf{4}-\mathbf{6}, \mathbf{8}$, and $\mathbf{1 0 - 1 3}$ were mea-

(41) Coe, B. J.; Foxon, S. P.; Harper, E. C.; Helliwell, M.; Raftery, J.; Swanson, C. A.; Brunschwig, B. S.; Clays, K.; Franz, E.; Garín, J.; Orduna, J.; Horton, P. N.; Hursthouse, M. B. J. Am. Chem. Soc. 2010, 132, 1706-1723. sured in acetonitrile solutions by using the HRS technique 28,29,31 $^{2}$ with a $800 \mathrm{~nm} \mathrm{Ti}^{3+}$ :sapphire laser, and the results are collected in Table 5, together with the previously published data for $\mathbf{1}$, 3, 7, and 9. ${ }^{15}$ This wavelength was chosen originally because the latter complexes do not absorb strongly at either $800 \mathrm{~nm}$ or at the second harmonic $(\mathrm{SH})$ of $400 \mathrm{~nm}$, their MLCT bands falling conveniently between these wavelengths. However, several of the new, extended chromophores do show intense ILCT bands near $400 \mathrm{~nm}$ (Table 2, Figures 2 and 3), and the results obtained for these salts are hence strongly affected by resonance enhancement. In order to obtain additional data, we have therefore also used a $1064 \mathrm{~nm} \mathrm{Nd}^{3+}$ :YAG laser to study these compounds, and the results are included in Table 5. A benefit of using a 800 or $1064 \mathrm{~nm}$ laser is the avoidance of any contributions due to two-photon luminescence. All of the $\mathrm{Ru}^{\mathrm{II}}$ complexes emit at wavelengths above $670 \mathrm{~nm}$ (Table 5), with negligible emission below about $600 \mathrm{~nm}$. In contrast, the $\mathrm{Fe}^{\mathrm{II}}$ complexes are predictably nonemissive at room temperature. Within the series $\mathbf{1}, \mathbf{2}$, and $\mathbf{5}$, the $\lambda_{\mathrm{em}}$ values red-shift steadily, consistent with the trend in the MLCT absorption maxima. However, the emission intensity is significantly weaker for $\mathbf{5}$ in comparison with those for $\mathbf{1}$ and $\mathbf{2}$. Comparison of the $\lambda_{\mathrm{em}}$ data for $\mathbf{3}$ and $\mathbf{4}$ also reveals a red shift, while the emission band for $\mathbf{6}$ falls between those of $\mathbf{2}$ and $\mathbf{4}$. The experimentally determined $\beta$ values for each operating wavelength are based on the assumption of a single tensor component, $\beta_{x x x}$, and are derived from the total HRS intensity $\left\langle\beta_{\mathrm{HRS}}{ }^{2}\right\rangle$ by using the equation

$$
\left\langle\beta_{\mathrm{HRS}}^{2}\right\rangle=\left(\frac{8}{35}+\frac{16}{105}\right) \beta_{x x x}^{2}=\left(\frac{8}{21}\right) \beta_{x x x}{ }^{2}
$$

While the effects of resonance preclude a detailed analysis of the HRS results, some trends are apparent. First, extending the ligand conjugated systems leads to steadily increasing $\beta$ values in all instances except for the $N$-phenylated pair $\mathbf{3}$ and $\mathbf{4}$ when studied at $800 \mathrm{~nm}$ (Table 5). It is well established that $\beta$ responses normally increase as conjugated systems are extended, ${ }^{1}$ although the very large values obtained for $\mathbf{2}$ and 4-6 can be attributed in large part to resonance enhancement. The second trend that emerges from the collected data is that the Ru-based chromophores give larger $\beta$ values than their $\mathrm{Fe}$ analogues in all cases except for the $\mathrm{Me}_{2} \mathrm{bbpe}^{2+}$-containing pair $\mathbf{2}$ and $\mathbf{8}$ when studied at $800 \mathrm{~nm}$. However, the $\beta_{x x x, 1064}$ value for $\mathbf{2}$ is about 3 times that for $\mathbf{8}$. Comparison within the Febased series 8, 10, and $\mathbf{1 2}$ does not reveal the steady increases in $\beta_{x x x, 800}$ that might be expected on the basis of the decreasing 
Table 5. MLCT Absorption, Luminescence, and HRS Data for Complex Salts 1-13 in Acetonitrile

\begin{tabular}{|c|c|c|c|c|}
\hline salt $\left(M / L^{A}\right)$ & $\lambda_{\max }, \mathrm{nm}$ & $\lambda_{\mathrm{em}}, \mathrm{nm}^{\mathrm{a}}$ & $\beta_{x x x, 800,}, 10^{-30}$ esu & $\beta_{x x x, 1064,}{ }^{c} 10^{-30}$ esu \\
\hline $1\left(\mathrm{Ru} / \mathrm{Me}_{2} \mathrm{qpy}^{2+}\right)^{d}$ & 486,394 & 671 & $170 \pm 21$ & $e$ \\
\hline $2\left(\mathrm{Ru} / \mathrm{Me}_{2} \mathrm{bbpe}^{2+}\right)$ & 506,428 & 712 & $190 \pm 35$ & $850 \pm 160$ \\
\hline $3\left(\mathrm{Ru} / \mathrm{Ph}_{2} \mathrm{qpy}^{2+}\right)^{d}$ & 496,410 & 685 & $270 \pm 35$ & $e$ \\
\hline $5\left(\mathrm{Ru} / \mathrm{Me}_{2} \mathrm{bbpb}^{2+}\right)$ & 513,428 & $764^{f}$ & $1200 \pm 400$ & $3100 \pm 700$ \\
\hline $6\left(\mathrm{Ru} / \mathrm{Me}_{2} \mathrm{bbpvb}^{2+}\right)$ & 501 & 718 & $1500 \pm 300$ & $5600 \pm 1300$ \\
\hline $7\left(\mathrm{Fe} / \mathrm{Me}_{2} \mathrm{qpy}^{2+}\right)^{d}$ & 574,440 & $g$ & $78 \pm 10$ & $\begin{array}{c}e \\
e\end{array}$ \\
\hline $8\left(\mathrm{Fe} / \mathrm{Me}_{2} \mathrm{bbpe}^{2+}\right)$ & 591,468 & $g$ & $320 \pm 110$ & $275 \pm 110$ \\
\hline $11\left(\mathrm{Fe} / \mathrm{Me}_{2} \mathrm{bbpb}^{2+}\right)$ & 600,475 & $g$ & $340 \pm 180$ & $340 \pm 200$ \\
\hline $12\left(\mathrm{Fe} /(2,4-\mathrm{DNPh})_{2} \mathrm{bbpe}^{2+}\right)$ & 611,495 & $g$ & $145 \pm 40$ & $400 \pm 140$ \\
\hline $13\left(\mathrm{Fe} / \mathrm{Me}_{2} \mathrm{bbpvb}^{2+}\right)$ & 581,458 & $g$ & $660 \pm 130$ & $700 \pm 200$ \\
\hline
\end{tabular}

${ }^{a}$ Emission maximum upon excitation into the MLCT bands ( $\lambda_{\mathrm{ex}} 480 \mathrm{~nm}$ for $\mathbf{2}$ and $\mathbf{5}, 514 \mathrm{~nm}$ for $\mathbf{4}$, and $510 \mathrm{~nm}$ for $\left.\mathbf{6}\right)$, with ca. $10^{-5} \mathrm{M}$ solutions; dilution produced no changes in the $\lambda_{\mathrm{em}}$ values. ${ }^{b}$ First hyperpolarizability measured by using a $800 \mathrm{~nm} \mathrm{Ti}{ }^{3+}$ :sapphire laser. ${ }^{c}$ First hyperpolarizability measured by using a $1064 \mathrm{~nm} \mathrm{Nd}^{3+}$ :YAG laser. The quoted cgs units (esu) can be converted into SI units $\left(\mathrm{C}^{3} \mathrm{~m}^{3} \mathrm{~J}^{-2}\right)$ by dividing by a factor of 2.693 $\times 10^{20} .{ }^{d}$ Data taken from ref $15 .{ }^{e}$ Not measured. ${ }^{f}$ Relatively weak signal. ${ }^{g}$ Not observed.

MLCT energies or previous studies with related 1D complexes. ${ }^{42}$ In contrast, $\mathbf{8}$ does show the smallest $\beta_{x x x, 1064}$ value of these three compounds.

Stark Spectroscopic Studies. Complex salts 2B, 4-6, 8B, 10, 11, 12B, and 13 have been studied by Stark spectroscopy ${ }^{33}$ in butyronitrile glasses at $77 \mathrm{~K}$, and the results are presented in Table 6, together with the previously published data for $\mathbf{1 , 3}, \mathbf{7}$, and 9. ${ }^{15}$ Gaussian fitting of the MLCT absorption spectra using three or four curves was necessary to successfully model the Stark data. It is likely that the ILCT transitions in the extended chromophores, especially 5, 6, 11, and 13, will contribute substantially to their NLO responses. However, these bands could be analyzed only for $\mathbf{6}$ and $\mathbf{1 3}$ because the shortwavelength operational cutoff of our Stark spectrometer is about $350 \mathrm{~nm}$. For both $\mathbf{6}$ and 13, the entire ICT region was treated as one. In the case of $\mathbf{6}$, strong overlap between the MLCT and ILCT bands means that it is not possible to fully resolve the two types of transition and a total of eight curves was used. In contrast, for $\mathbf{1 3}$ there is a relatively good degree of separation between the MLCT and ILCT bands; three curves were then used for the former and four for the latter, with some overlap even so. The resulting data for $\mathbf{6}$ and $\mathbf{1 3}$ are included in Table 6. Representative absorption spectra and electroabsorption spectra in the MLCT region for the complex salts $\mathbf{2 B}, \mathbf{5}$, and 8B are shown in Figure 6, while the full ICT region spectra for for $\mathbf{6}$ and $\mathbf{1 3}$ are shown in Figure 7. The MLCT region spectra for all of the other new complex salts are provided as Supporting Information (Figures S1 and S2).

As noted previously for $\mathbf{1}$ and $\mathbf{3}$, for each of the Ru complexes a relatively low intensity Gaussian curve was placed to low energy in order to fit the Stark signal satisfactorily; these curves may correspond with spin-forbidden transitions to triplet MLCT excited states. ${ }^{43}$ For $\mathbf{1}, \mathbf{3}, \mathbf{7}$, and $\mathbf{9}$, only two of the three Gaussian curves used to fit the transitions to singlet excited states contribute significantly to the observed Stark signals; therefore, the data obtained for the other functions are not quoted. However, for all of the new complex salts the latter are significant contributors; thus, the corresponding data are included in Table 6.

(42) (a) Coe, B. J.; Harris, J. A.; Harrington, L. J.; Jeffery, J. C.; Rees, L. H.; Houbrechts, S.; Persoons, A. Inorg. Chem. 1998, 37, 33913399. (b) Coe, B. J.; Harries, J. L.; Harris, J. A.; Brunschwig, B. S.; Coles, S. J.; Light, M. E.; Hursthouse, M. B. Dalton Trans. 2004, $2935-2942$.

(43) Kober, E. M.; Meyer, T. J. Inorg. Chem. 1982, 21, 3967-3977.
For the MLCT bands, the $E_{\max }$ values for the two most intense Gaussian curves fitted to the absorption spectra at $77 \mathrm{~K}$ in butyronitrile are generally decreased in comparison with the data recorded in acetonitrile solutions (Table 3). In almost every instance, the data obtained at $77 \mathrm{~K}$ show the same trends as those observed at room temperature: i.e., the red-shifting effects arising from extending the conjugated systems, replacing $\mathrm{Ru}$ with Fe, or replacing $N$-methyl with $N$-phenyl/2,4-dinitrophenyl substituents (see above). In order to analyze the $f_{\text {os }}$ and $\mu_{12}$ data, it is appropriate to consider the totals for the various components, since the superposition of these gives the overall absorption profiles. The total $f_{\text {os }}$ and $\mu_{12}$ values measured at 77 $\mathrm{K}$ show trends that generally correlate with those observed in the $\epsilon$ values in acetonitrile solutions (Table 3). Thus, the MLCT intensities increase when the conjugated systems are extended or when a $N$-methyl is replaced with a $N$-phenyl/2,4-dinitrophenyl substituent. Also, the Fe complexes show lower total $f_{\text {os }}$ and $\mu_{12}$ values in comparison with their $\mathrm{Ru}$ analogues. This latter observation also applies to the combined MLCT and ILCT data for 6 and 13.

The derived adiabatic and diabatic dipole moment changes ( $\Delta \mu_{12}$ and $\Delta \mu_{\mathrm{ab}}$, respectively) for the MLCT bands cover a broad range of ca. 5-34 D, with the largest being measured for one of the Gaussian components for 13. For the purpose of analyzing and comparing these parameters, it is reasonable to consider average values because the fitted Gaussian components represent hypothetical ICT transitions that are codirectional but not additive in terms of their dipole moment changes. Both $\Delta \mu_{12}$ and $\Delta \mu_{\mathrm{ab}}$ generally increase in a logical fashion as the conjugated systems are extended: for example, on moving along the $\mathrm{Ru}$ series $\mathbf{1} \rightarrow \mathbf{2 B} \rightarrow \mathbf{5}$. Except for the pair $\mathbf{1}$ and $\mathbf{3}$, a pattern of increasing $\Delta \mu_{12}$ and $\Delta \mu_{\mathrm{ab}}$ on replacing $N$-methyl with $N$-phenyl substituents is also observed, and steady increases occur on moving along the Fe series $\mathbf{8 B} \rightarrow \mathbf{1 0} \rightarrow \mathbf{1 2 B}$. Overall, there is no clear trend in the dipole moment changes on varying the metal center. Since they derive directly from these data, the average delocalized and localized electron-transfer distances $\left(r_{12}\right.$ and $r_{\mathrm{ab}}$, respectively) show the same trends, with $r_{\mathrm{ab}}$ exceeding $5 \AA$ in several instances and being as high as $7 \AA$ for $\mathbf{1 3}$. Although the parameters $c_{\mathrm{b}}{ }^{2}$ and $H_{\mathrm{ab}}$ might be expected to decrease steadily with extension of the conjugated systems, the data obtained do not provide convincing evidence for such behavior, especially since the estimated errors on the $c_{\mathrm{b}}{ }^{2}$ values are very large. 
Table 6. ICT Absorption and Stark Spectroscopic Data for Complex Salts 1-13

\begin{tabular}{|c|c|c|c|c|c|c|c|c|c|c|c|c|c|c|c|}
\hline salt $\left(M / L^{A}\right)$ & $\begin{array}{l}\nu_{\max }{ }^{a}{ }^{a} \\
\mathrm{~cm}^{-1}\end{array}$ & $\begin{array}{c}\lambda_{\max }{ }^{a} \\
n m\end{array}$ & $\begin{array}{c}E_{\max }{ }^{a} \\
\mathrm{eV}\end{array}$ & $f_{\text {os }}$ & $\begin{array}{c}\mu_{12,}^{b} \\
\mathrm{D}\end{array}$ & $\begin{array}{c}\Delta \mu_{12,}{ }^{c} \\
D\end{array}$ & $\begin{array}{c}\Delta \mu_{\mathrm{ab}}{ }^{d} \\
\mathrm{D}\end{array}$ & $\begin{array}{c}r_{12}, e \\
\AA\end{array}$ & $\stackrel{r_{a b}, f}{\AA}$ & $c_{0}^{2 g}$ & $\begin{array}{c}H_{\mathrm{ab},}{ }^{\mathrm{h}}{ }^{-1} \\
10^{3} \mathrm{~cm}^{-1}\end{array}$ & $\begin{array}{c}\beta_{0,}{ }^{i} \\
10^{-30} \text { esu } \\
\end{array}$ & $\underset{10^{-30}}{\sum \beta_{0}}$ esu & $N^{j}$ & $\begin{array}{c}\beta_{\mathrm{EN},}{ }^{k} \\
10^{-32} \mathrm{esu}\end{array}$ \\
\hline \multirow[t]{2}{*}{$1\left(\mathrm{Ru} / \mathrm{Me}_{2} \mathrm{qpy}{ }^{2+}\right)^{l}$} & 17359 & 576 & 2.15 & 0.002 & 0.5 & 12.9 & 12.9 & 2.7 & 2.7 & 0.00 & 0.7 & 1 & 68 & 72 & 11.1 \\
\hline & 20149 & 496 & 2.50 & 0.17 & 4.2 & 7.9 & 11.6 & 1.6 & 2.4 & 0.16 & 7.4 & 26 & & & \\
\hline \multirow[t]{4}{*}{ 2B $\left(\mathrm{Ru} / \mathrm{Me}_{2} \mathrm{bbpe}^{2+}\right)$} & 17421 & 574 & 2.16 & 0.07 & 2.9 & 17.4 & 18.4 & 3.6 & 3.8 & 0.02 & 2.8 & 37 & 191 & 84 & 24.8 \\
\hline & 19115 & 523 & 2.37 & 0.32 & 6.0 & 13.0 & 17.7 & 2.7 & 3.7 & 0.13 & 6.5 & 98 & & & \\
\hline & 21132 & 473 & 2.62 & 0.19 & 4.3 & 9.6 & 13.0 & 2.0 & 2.7 & 0.13 & 7.1 & 30 & & & \\
\hline & 23309 & 429 & 2.89 & 0.16 & 3.8 & 12.9 & 14.9 & 2.7 & 3.1 & 0.07 & 5.9 & 26 & & & \\
\hline $3\left(\mathrm{Ru} / \mathrm{Ph}_{2} \mathrm{qpy}^{2+}\right)^{l}$ & 24553 & 407 & 3.04 & 0.51 & 6.7 & 13.5 & 19.0 & 2.8 & 4.0 & 0.14 & 8.6 & 76 & & & \\
\hline \multirow[t]{4}{*}{$4\left(\mathrm{Ru} / \mathrm{Ph}_{2} \mathrm{bbpe}^{2+}\right)$} & 17664 & 566 & 2.19 & 0.27 & 5.7 & 22.8 & 25.4 & 4.8 & 5.3 & 0.05 & 4.0 & 181 & 586 & 120 & 44.6 \\
\hline & 18712 & 534 & 2.32 & 0.39 & 6.7 & 15.9 & 20.7 & 3.3 & 4.3 & 0.12 & 6.0 & 146 & & & \\
\hline & 20164 & 496 & 2.50 & 0.32 & 5.8 & 15.7 & 19.5 & 3.3 & 4.1 & 0.10 & 6.0 & 101 & & & \\
\hline & 22100 & 452 & 2.74 & 0.52 & 7.1 & 21.2 & 25.5 & 4.4 & 5.3 & 0.08 & 6.1 & 158 & & & \\
\hline $5\left(\mathrm{Ru} / \mathrm{Me}_{2} \mathrm{bbpb}^{2+}\right)$ & 17180 & 582 & 2.13 & 0.12 & 3.7 & 20.6 & 21.9 & 4.3 & 4.6 & 0.03 & 2.8 & 73 & 451 & 96 & 47.9 \\
\hline \multirow{6}{*}{$6\left(\mathrm{Ru} / \mathrm{Me}_{2} \mathrm{bbpvb}^{2+}\right)$} & 20978 & 477 & 2.60 & 0.49 & 7.1 & 16.4 & 21.7 & 3.4 & 4.5 & 0.12 & 6.8 & 141 & & & \\
\hline & 22896 & 437 & 2.84 & 1.28 & 10.9 & 18.1 & 28.4 & 3.8 & 5.9 & 0.18 & 8.8 & 313 & & & \\
\hline & 24396 & 410 & 3.02 & 1.00 & 9.4 & 4.7 & 19.4 & 1.0 & 4.0 & 0.38 & 11.8 & 53 & & & \\
\hline & 25326 & 395 & 3.14 & 0.68 & 7.6 & 6.0 & 16.3 & 1.3 & 3.4 & 0.31 & 11.8 & 41 & & & \\
\hline & 26239 & 381 & 3.25 & 1.21 & 9.9 & 5.1 & 20.5 & 1.1 & 4.3 & 0.37 & 12.7 & 56 & & & \\
\hline & 27602 & 362 & 3.42 & 0.57 & 6.6 & 0.0 & 13.3 & 0.0 & 2.8 & 0.50 & 13.8 & 0 & & & \\
\hline \multirow[t]{2}{*}{$7\left(\mathrm{Fe} / \mathrm{Me}_{2} \mathrm{qpy}^{2+}\right)^{l}$} & 17225 & 581 & 2.14 & 0.16 & 4.4 & 6.1 & 10.6 & 1.3 & 2.2 & 0.22 & 7.1 & 30 & 86 & 72 & 14.1 \\
\hline & 22531 & 444 & 2.79 & 0.35 & 5.8 & 11.2 & 16.1 & 2.3 & 3.4 & 0.15 & 8.1 & 56 & & & \\
\hline \multirow{3}{*}{$\mathbf{8 B}\left(\mathrm{Fe} / \mathrm{Me}_{2} \mathrm{bbpe}^{2+}\right)$} & 16292 & 614 & 2.02 & 0.11 & 3.9 & 10.1 & 12.7 & 2.1 & 2.7 & 0.10 & 4.9 & 44 & 197 & 84 & 25.6 \\
\hline & 16534 & 605 & 2.05 & 0.26 & 5.8 & 9.7 & 15.1 & 2.0 & 3.2 & 0.08 & 6.4 & 91 & & & \\
\hline & 20486 & 488 & 2.54 & 0.18 & 4.3 & 18.5 & 20.4 & 3.9 & 4.3 & 0.05 & 4.4 & 62 & & & \\
\hline \multirow[t]{2}{*}{$9\left(\mathrm{Fe} / \mathrm{Ph}_{2} \mathrm{qpy}^{2+}\right)^{l}$} & 16950 & 590 & 2.10 & 0.16 & 4.5 & 7.4 & 11.6 & 1.5 & 2.4 & 0.18 & 6.5 & 39 & 121 & 108 & 10.8 \\
\hline & 21675 & 461 & 2.69 & 0.37 & 6.1 & 13.9 & 18.4 & 2.9 & 3.8 & 0.12 & 7.1 & 82 & & & \\
\hline \multirow[t]{7}{*}{$13\left(\mathrm{Fe} / \mathrm{Me}_{2} \mathrm{bbpvb}^{2+}\right)$} & 16569 & 604 & 2.05 & 0.42 & 7.4 & 10.0 & 17.8 & 2.1 & 3.7 & 0.22 & 6.9 & 150 & $836(450)^{m}$ & 132 & $55.1(29.7)^{m}$ \\
\hline & 17833 & 561 & 2.21 & 0.16 & 4.4 & 8.8 & 12.5 & 1.8 & 2.6 & 0.15 & 6.3 & 41 & & & \\
\hline & 20675 & 484 & 2.56 & 0.46 & 6.9 & 30.7 & 33.7 & 6.4 & 7.0 & 0.04 & 4.2 & 259 & & & \\
\hline & 23818 & 420 & 2.95 & 0.86 & 8.8 & 5.8 & 18.5 & 1.2 & 3.9 & 0.34 & 11.3 & 60 & & & \\
\hline & 24635 & 406 & 3.05 & 1.44 & 11.2 & 10.9 & 24.9 & 2.3 & 5.2 & 0.28 & 11.1 & 170 & & & \\
\hline & 25561 & 391 & 3.17 & 1.18 & 9.9 & 8.9 & 21.7 & 1.8 & 4.5 & 0.30 & 11.7 & 101 & & & \\
\hline & 26759 & 374 & 3.32 & 0.97 & 8.8 & 6.7 & 18.8 & 1.4 & 3.9 & 0.32 & 12.5 & 55 & & & \\
\hline
\end{tabular}

\footnotetext{
${ }^{a}$ Maxima for Gaussian functions used to fit to spectral data recorded in butyronitrile glass at $77 \mathrm{~K} .{ }^{b}$ Calculated using eq $2 ; f_{\text {os }}$ obtained from $(4.60 \times$ $\left.10^{-9} \mathrm{M} \mathrm{cm}^{2}\right) \epsilon_{\max } \times f w_{1 / 2}$, where $\epsilon_{\max }$ is the maximal molar extinction coefficient and $f w_{1 / 2}$ is the full width at half height (in wavenumbers). ${ }^{c}$ Calculated from $f_{\text {int }} \Delta \mu_{12}$ using $f_{\text {int }}=1.33$. ${ }^{d}$ Calculated from eq $1 .{ }^{e}$ Delocalized electron-transfer distance calculated from $\Delta \mu_{12} / e$. ${ }^{f}$ Effective (localized) electron-transfer distance calculated from $\Delta \mu_{\mathrm{ab}} / e .{ }^{g}$ Calculated from eq $3 .{ }^{h}$ Calculated from eq $4 .{ }^{i}$ Calculated from eq $5 .{ }^{j}$ The total number of $\pi$-bonding electrons (excluding metal d-electrons) in the complex cation. ${ }^{k}$ Electron-normalized off-resonant first hyperpolarizability, defined as $\sum \beta_{0} / N^{3 / 2} \cdot{ }^{50}{ }^{l}$ Data taken from ref $15 .{ }^{m}$ The total contribution associated with (largely) only the MLCT transitions is in brackets for purposes of comparison with the other compounds.
}

As in our previous studies, ${ }^{15}$ we have used the standard twostate model (eq 5$)^{44}$ to estimate static first hyperpolarizabilities from the Stark data for the transitions to dipolar ICT excited states. Although metal tris-chelate complexes have octupolar ground-state structures, ${ }^{7}$ their NLO responses are clearly associated largely with dipolar ICT transitions. In this context, it is also important to note that other two-state descriptions are also available, which would alter the Stark-derived $\beta_{0}$ values

(44) (a) Oudar, J. L.; Chemla, D. S. J. Chem. Phvs. 1977, 66, 2664-2668. (b) Oudar, J. L. J. Chem. Phys. 1977, 67, 446-457. quoted here by a constant factor of 0.5 or $2 .{ }^{45}$ These data may hence be used primarily as a means to reveal structure-activity relationships involving all contributing ICT transitions without any interference from variable resonance effects, rather than necessarily revealing the absolute magnitudes of $\beta_{0}$. Even so, it is remarkable that our previous studies with dipolar $\mathrm{Ru}^{\mathrm{II}}$ ammine complexes demonstrate that using the two-state equation (5) with a prefactor of 3 (the "perturbation series convention") ${ }^{45}$

(45) Willetts, A.; Rice, J. E.; Burland, D. M.; Shelton, D. P. J. Chem. Phvs. 1992, 97, 7590-7599. 


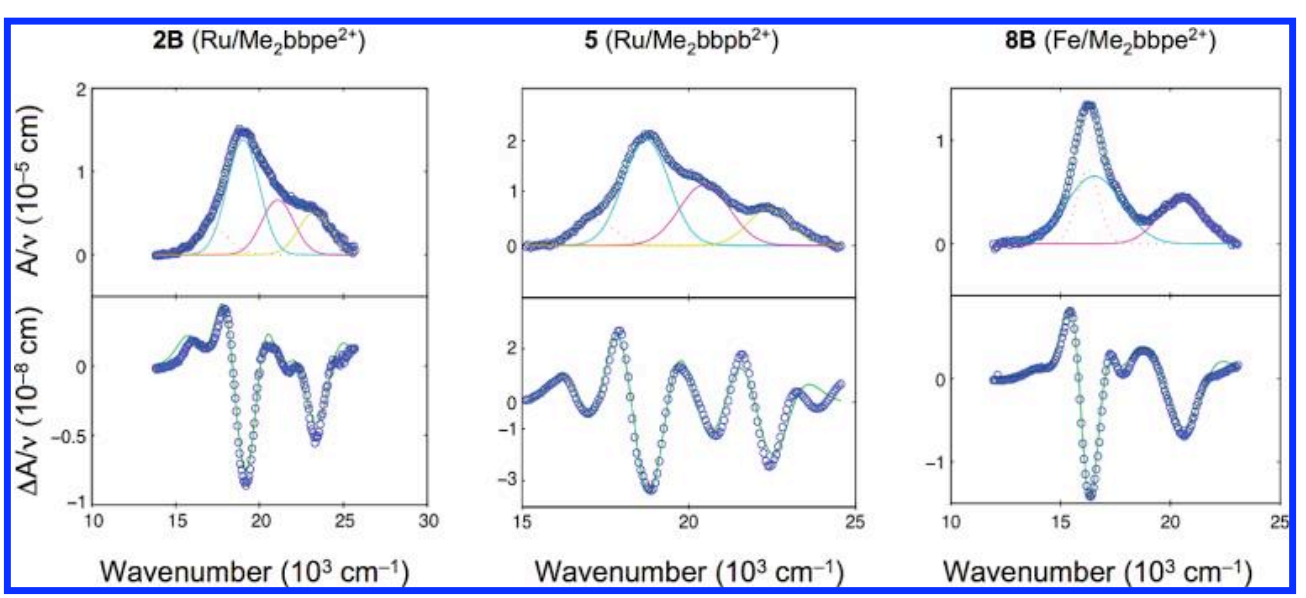

Figure 6. MLCT and Stark spectra with calculated fits for complex salts $\mathbf{2 B}, \mathbf{5}$, and $\mathbf{8 B}$ in an external electric field of $4.74 \times 10^{7} \mathrm{~V} \mathrm{~m} \mathrm{~m}^{-1}$ : (top panels) absorption spectra illustrating Gaussian curves used in data fitting; (bottom panels) electroabsorption spectra, with experimental data shown in blue and fits in green according to the Liptay equation. ${ }^{33}$

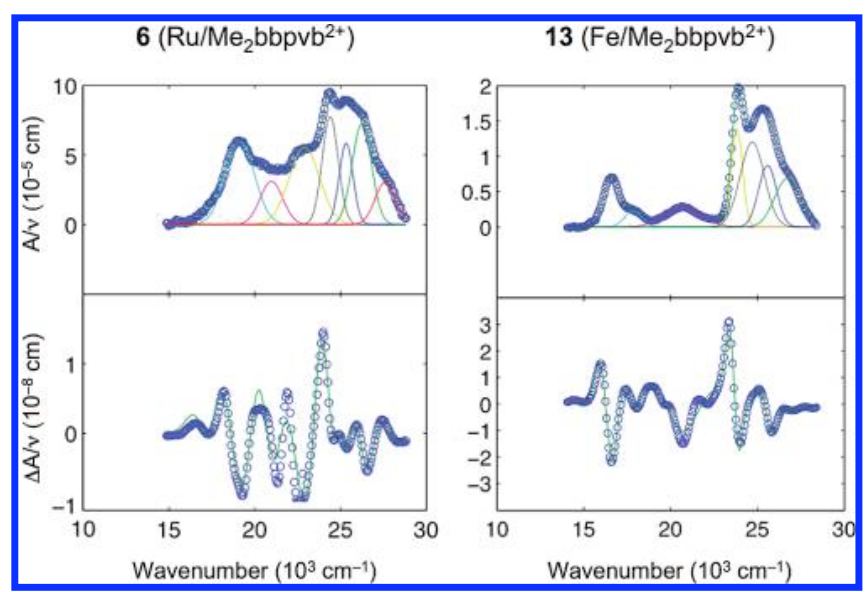

Figure 7. ICT and Stark spectra with calculated fits for complex salts 6 and $\mathbf{1 3}$ in an external electric field of $5.36 \times 10^{7} \mathrm{~V} \mathrm{~m}$ m $^{-1}$ : (top panels) absorption spectra illustrating Gaussian curves used in data fitting; (bottom panels) electroabsorption spectra, with experimental data shown in blue and fits in green according to the Liptay equation. ${ }^{33}$

often gives excellent quantitative agreement with the $\beta_{0}$ values determined via HRS with a $1064 \mathrm{~nm}$ laser. ${ }^{32 \mathrm{~b}}$

We have shown in several previous studies that while using deconvolution is required to give satisfactory fits to the Stark data, the total $\left[\beta_{0}\right]_{\text {ICT }}$ values that arise from such treatment are generally very similar to those obtained without deconvolution. ${ }^{41,46}$ These precedents confirm the reliability of this indirect approach to deriving $\beta_{0}$ responses. While it is probably of little value to consider the data for the individual Gaussian components in detail due to the uncertainties involved in the spectral deconvolution, the resulting total $\beta_{0}$ responses do merit further comments. First, sequential and dramatic increases are observed on extending the conjugated systems in moving along the Ru series $\mathbf{1} \rightarrow \mathbf{2 B} \rightarrow \mathbf{5}$ or the Fe series $\mathbf{7} \rightarrow \mathbf{8 B} \rightarrow \mathbf{1 1}$, with the $\mathrm{Me}_{2} \mathrm{bbpb}^{2+}{ }_{\text {-containing chromophores having estimated total }}$ MLCT-associated $\beta_{0}$ values about 6 times larger than those of their previously reported qpy-based counterparts. Moving from $\mathbf{1 1}$ to $\mathbf{1 3}$ does not change $\sum \beta_{0}$ significantly when considering only the MLCT-based contributions, but the total $\beta_{0}$ response derived for $\mathbf{1 3}$ is increased almost 2-fold when the ILCT

(46) Coe, B. J.; Foxon, S. P.; Harper, E. C.; Harris, J. A.; Helliwell, M.; Raftery, J.; Asselberghs, I.; Clays, K.; Franz, E.; Brunschwig, B. S.; Fitch, A. G. Dves Pigments 2009, 82, 171-186. contributions are included. Large increases in $\Sigma \beta_{0}$ also accompany the insertion of $(E)$-vinyl bridges into the $N$-phenyl chromophores, i.e. on moving from $\mathbf{3}$ to $\mathbf{4}$ and from $\mathbf{9}$ to $\mathbf{1 0 .}$

The second trend observed in the $\Sigma \beta_{0}$ values is that these increase substantially (ca. $1.5-3$ times) on replacing $N$-methyl with more strongly electron-accepting $N$-phenyl substituents. Both of these trends evident in the $\Sigma \beta_{0}$ values for $\mathbf{1 - 1 3}$ reflect those we have observed previously when analyzing the MLCT bands for related 1D metallochromophores. ${ }^{21,32 b, 40,42 b}$ Although the $N$-2,4-dinitrophenyl substituent is an even more powerful electron acceptor according to the MLCT energies and ligandbased reduction potentials (see above), the total $\beta_{0}$ response derived for $\mathbf{1 2 B}$ is possibly slightly smaller than that for $\mathbf{1 0}$. This result can be attributed largely to the decreased MLCT transition intensities caused by diminished $\pi$-orbital overlap due to twisting of the 2,4-DNPh group with respect to the rest of the conjugated systems; this effect is especially evident in the $f_{\text {os }}$ and $\mu_{12}$ values measured at $77 \mathrm{~K}$ (Table 6). It is, however, worth noting that our previous Stark studies have shown that replacing $N$-Ph with 2,4 -DNPh substituents leads to increases in $\beta_{0}$, in both metal complexes ${ }^{42 \mathrm{~b}}$ and purely organic chromophores. ${ }^{47}$ Also in contrast with the HRS results (see above), the estimated $\sum \beta_{0}$ values for $\mathbf{1 - 1 3}$ taken as a whole do not provide any clear indication that the nature of the metal center substantially affects the NLO responses. Although $\Sigma \beta_{0}$ appears to decrease considerably on replacing $\mathrm{Ru}$ with $\mathrm{Fe}$ in two instances (pairs 4/10 and 6/13), the differences are within the experimental error limits.

It is not appropriate to attempt direct, quantitative comparisons between HRS and Stark-derived $\beta$ values, because of the importance of resonance effects in HRS measurements and the potential uncertainty about the prefactor in eq 5 (see above). The challenges inherent in obtaining reliable $\beta_{0}$ data, especially from HRS studies, have been discussed previously. ${ }^{48}$ Unfortunately, it is not possible to use this technique to derive anything

(47) Coe, B. J.; Harris, J. A.; Asselberghs, I.; Wostyn, K.; Clays, K.; Persoons, A.; Brunschwig, B. S.; Coles, S. J.; Gelbrich, T.; Light, M. E.; Hursthouse, M. B.; Nakatani, K. Adv. Funct. Mater. 2003, 13, 347-357.

(48) Selected examples: (a) Woodford, J. N.; Wang, C. H.; Jen, A. K.-Y. Chem. Phys. 2001, 271, 137-143. (b) Di Bella, S. New J. Chem. 2002, 26, 495-497. (c) Tai, O. Y.-H.; Wang, C. H.; Ma, H.; Jen, A. K.-Y. J. Chem. Phys. 2004, 121, 6086-6092. (d) Campo, J.; Wenseleers, W.; Goovaerts, E.; Szablewski, M.; Cross, G. H. J. Phvs. Chem. C 2008, 112, 287-296. 
Table 7. Cubic NLO Parameters for Complex Salts $\mathbf{1 - 1 3}$ at $750 \mathrm{~nm}$

\begin{tabular}{|c|c|c|c|c|}
\hline salt $\left(M / L^{A}\right)$ & $\gamma_{\text {real }}, 10^{-36}$ esu & $\gamma_{\text {imag }}, 10^{-36} \mathrm{esu}$ & $|\gamma|, 10^{-36}$ esu & $\sigma_{2}, \mathrm{GM}^{a}$ \\
\hline $1\left(\mathrm{Ru} / \mathrm{Me}_{2} \mathrm{qpy}^{2+}\right)^{b}$ & $-4300 \pm 600$ & $220 \pm 30$ & $4300 \pm 600$ & $62 \pm 8$ \\
\hline $2\left(\mathrm{Ru} / \mathrm{Me}_{2} \mathrm{bbpe}^{2+}\right)$ & $-3000 \pm 1500$ & $2600 \pm 300$ & $4000 \pm 1500$ & $720 \pm 90$ \\
\hline $3\left(\mathrm{Ru} / \mathrm{Ph}_{2} \mathrm{qpy}^{2+}\right)^{b}$ & $-5800 \pm 1500$ & $420 \pm 100$ & $5800 \pm 1500$ & $120 \pm 25$ \\
\hline $4\left(\mathrm{Ru} / \mathrm{Ph}_{2} \mathrm{bbpe}^{2+}\right)$ & $-9000 \pm 1000$ & $4200 \pm 200$ & $9900 \pm 1000$ & $1200 \pm 50$ \\
\hline $5\left(\mathrm{Ru} / \mathrm{Me}_{2} \mathrm{bbpb}^{2+}\right)$ & $-12000 \pm 2000$ & $5200 \pm 600$ & $13100 \pm 2000$ & $1500 \pm 170$ \\
\hline $6\left(\mathrm{Ru} / \mathrm{Me}_{2} \mathrm{bbpvb}^{2+}\right)$ & $-24000 \pm 6000$ & $8900 \pm 900$ & $25600 \pm 6000$ & $2500 \pm 250$ \\
\hline $7\left(\mathrm{Fe} / \mathrm{Me}_{2} \mathrm{qpy}^{2+}\right)^{b}$ & $-7400 \pm 800$ & $48 \pm 15$ & $7400 \pm 800$ & $13 \pm 4$ \\
\hline $8\left(\mathrm{Fe} / \mathrm{Me}_{2} \mathrm{bbpe}^{2+}\right)$ & $-1700 \pm 1000$ & $1200 \pm 150$ & $2100 \pm 1000$ & $350 \pm 40$ \\
\hline $9\left(\mathrm{Fe} / \mathrm{Ph}_{2} \mathrm{qpy}^{2+}\right)^{b}$ & $-6400 \pm 1500$ & $13 \pm 6$ & $6400 \pm 1500$ & $3.6 \pm 2$ \\
\hline $10\left(\mathrm{Fe} / \mathrm{Ph}_{2} \mathrm{bbpe}^{2+}\right)$ & $-11000 \pm 2000$ & $2200 \pm 400$ & $11200 \pm 2000$ & $600 \pm 100$ \\
\hline $11\left(\mathrm{Fe} / \mathrm{Me}_{2} \mathrm{bbpb}^{2+}\right)$ & $-13000 \pm 4000$ & $5500 \pm 700$ & $14100 \pm 4000$ & $1500 \pm 200$ \\
\hline $12\left(\mathrm{Fe} /(2,4-\mathrm{DNPh})_{2} \mathrm{bbpe}^{2+}\right)$ & $-21000 \pm 2000$ & $950 \pm 120$ & $21000 \pm 2000$ & $265 \pm 30$ \\
\hline $13\left(\mathrm{Fe} / \mathrm{Me}_{2} \mathrm{bbpvb}^{2+}\right)$ & $-18000 \pm 3000$ & $5100 \pm 300$ & $18700 \pm 3000$ & $1400 \pm 100$ \\
\hline
\end{tabular}

${ }^{a} 1 \mathrm{GM}=10^{-50} \mathrm{~cm}^{4} \mathrm{~s}$ photon ${ }^{-1} \cdot{ }^{b}$ Data taken from ref 16.

more than resonance-enhanced $\beta$ values for compounds that display more than one ICT band. Therefore, the Stark-derived $\beta_{0}$ data we report for $\mathbf{1 - 1 3}$ give the most accurate description of the (relative) NLO responses of this series of chromophores. However, it should also be emphasized that the $\beta_{0}$ values for most of the new complex chromophores are only lower limits, since they do not account for contributions associated with the ILCT transitions. In $\mathrm{Ru}^{\mathrm{II}}$ ammine complexes of monodentate ligands related to $\mathrm{Me}_{2} \mathrm{bbpb}^{2+}$, the ILCT processes account for ca. $10-20 \%$ of the total estimated $\beta_{0}$ values. ${ }^{21,41}$ Given that the tris-chelate derivatives contain six individual $\pi$-conjugated "arms", the total ILCT contributions to $\beta_{0}$ will be larger when compared with related $1 \mathrm{D}$ or $2 \mathrm{D}$ systems. Nevertheless, the MLCT-associated responses achieved of ca. $(400-600) \times 10^{-30}$ esu for 4, 5, 10, and $\mathbf{1 1}$ are among the largest (off-resonance) quadratic NLO responses determined for metal-containing chromophores. Furthermore, the $\Sigma \beta_{0}$ values for $\mathbf{6}$ and $\mathbf{1 3}$ are possibly the highest yet recorded. These results serve to highlight the utility of the Stark-based approach, which is the only method by which such data for chromophores with relatively complicated ICT spectra may be obtained. In order to set these results in context, it is worth considering data obtained by applying the Stark-based approach to a benchmark chromophore, under the same experimental conditions and using eq 5. The salt $(E)-$ $4^{\prime}$-(dimethylamino)- $N$-methyl-4-stilbazolium (DAS) tosylate ${ }^{49}$ is the first organic NLO material to become commercialized (for $\mathrm{THz}$ wave generation via nonlinear frequency mixing). We have derived previously a $\beta_{0}$ value of $236 \times 10^{-30}$ esu for [DAS] $\mathrm{PF}_{6}{ }^{47}$ substantially smaller than most of those determined for the new compounds (Table 6).

It is well established that hyperpolarizabilities generally increase with the size of the $\pi$-conjugated system. ${ }^{1}$ In attempts to assess the extent to which quadratic NLO responses are also influenced by other factors and to probe their fundamental quantum limits, recent studies with purely organic dipolar chromophores have included electron-normalized off-resonant

(49) Selected examples: (a) Kawase, K.; Mizuno, M.; Sohma, S.; Takahashi, H.; Taniuchi, T.; Urata, Y.; Wada, S.; Tashiro, H.; Ito, H. Opt. Lett. 1999, 24, 1065-1067. (b) Kawase, K.; Hatanaka, T.; Takahashi, H.; Nakamura, K.; Taniuchi, T.; Ito, H. Opt. Lett. 2000, 25, 1714-1716. (c) Taniuchi, T.; Okada, S.; Nakanishi, H. Appl. Phvs. Lett. 2004, 95, 5984-5988. (d) Taniuchi, T.; Ikeda, S.; Okada, S.; Nakanishi, H. Jpn. J. Appl. Phvs. 2005, 44, L652-L654. (e) Schneider, A.; Neis, M.; Stillhart, M.; Ruiz, B.; Khan, R. U. A.; Günter, P. J. Opt. Soc. Am. B 2006, 23, 1822-1835. (f) Schneider, A.; Stillhart, M.; Günter, P. Opt. Express 2006, 14, 5376-5384. (g) Yang, Z.; Mutter, L.; Stillhart, M.; Ruiz, B.; Aravazhi, S.; Jazbinsek, M.; Schneider, A.; Gramlich, V.; Günter, P. Adv. Funct. Mater. 2007, 17, 2018-2023.

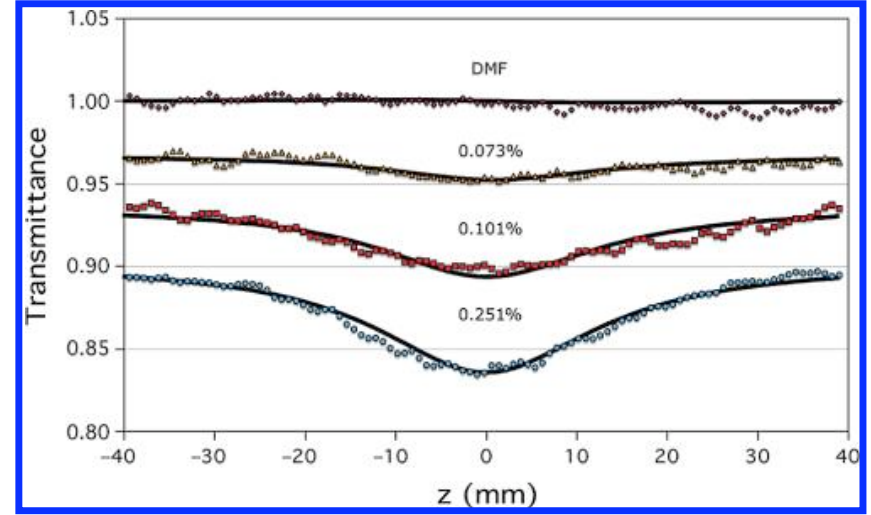

Figure 8. Examples of open-aperture Z-scans on $\mathbf{1 1}$ in DMF solutions at $750 \mathrm{~nm}$. Full lines denote theoretically computed curves.

first hyperpolarizabilities $\beta_{\mathrm{EN}},{ }^{50}$ which are derived by considering the total number of $\pi$-bonding electrons. We have therefore calculated $\beta_{\mathrm{EN}}$ values for $\mathbf{1 - 1 3}$, and these are included in Table 6. These data confirm substantial enhancements in the NLO response on moving along the series $\mathbf{1} \rightarrow \mathbf{2 B} \rightarrow \mathbf{5}$ and $\mathbf{7} \rightarrow \mathbf{8 B}$ $\rightarrow$ 11. Replacing $\mathrm{R}=\mathrm{Me}$ with $\mathrm{R}=\mathrm{Ph}$ gives lower $\beta_{\mathrm{EN}}$ values for the qpy-based species but higher values for their bbpe-based counterparts. The series $\mathbf{8 B} \rightarrow \mathbf{1 0} \rightarrow \mathbf{1 2 B}$ shows an increase in $\beta_{\mathrm{EN}}$ on moving from $\mathrm{R}=\mathrm{Me}$ to $\mathrm{R}=\mathrm{Ph}$, but then a decrease for the 2,4-DNPh-containing complex. The application of this approach to the Stark-derived $\beta_{0}$ values for stilbazolium and closely related organic chromophores ${ }^{46,47}$ also indicates that the $\beta_{\mathrm{EN}}$ responses of $N$-Ph species are larger than those of their 2,4-DNPh analogues. ${ }^{51}$

Two-Photon Absorption Studies. The new complex salts 2, 4-6, 8, and 10-13 were investigated in DMF solutions by using the Z-scan technique ${ }^{34,35}$ at a laser wavelength of $750 \mathrm{~nm}$, to afford the real and imaginary parts of $\gamma$ together with the 2PA cross sections $\sigma_{2}$. The results are collected in Table 7, along with the data published previously for $1,3,7$, and $9 .{ }^{16}$ Figure 8 shows an example set of open-aperture Z-scans on $\mathbf{1 1}$ showing the concentration dependence of the dip due to 2PA. The curves are shifted due to the presence of some one-photon absorption in solutions of $\mathbf{1 1}$ at $750 \mathrm{~nm}$.

All of the complex salts $\mathbf{1 - 1 3}$ show relatively strong negative refractive nonlinearity, as indicated by their $\gamma_{\text {real }}$ values (Table

(50) (a) Kuzyk, M. G. Phvs. Rev. Lett. 2000, 85, 1218-1221. (b) Kuzyk, M. G. Phys. Rev. Lett. 2003, 90, 039902. (c) Tripathy, K.; Pérez Moreno, J.; Kuzyk, M. G.; Coe, B. J.; Clays, K.; Myers Kelley, A. J. Chem. Phys. 2004, 121, 7932-7945. 


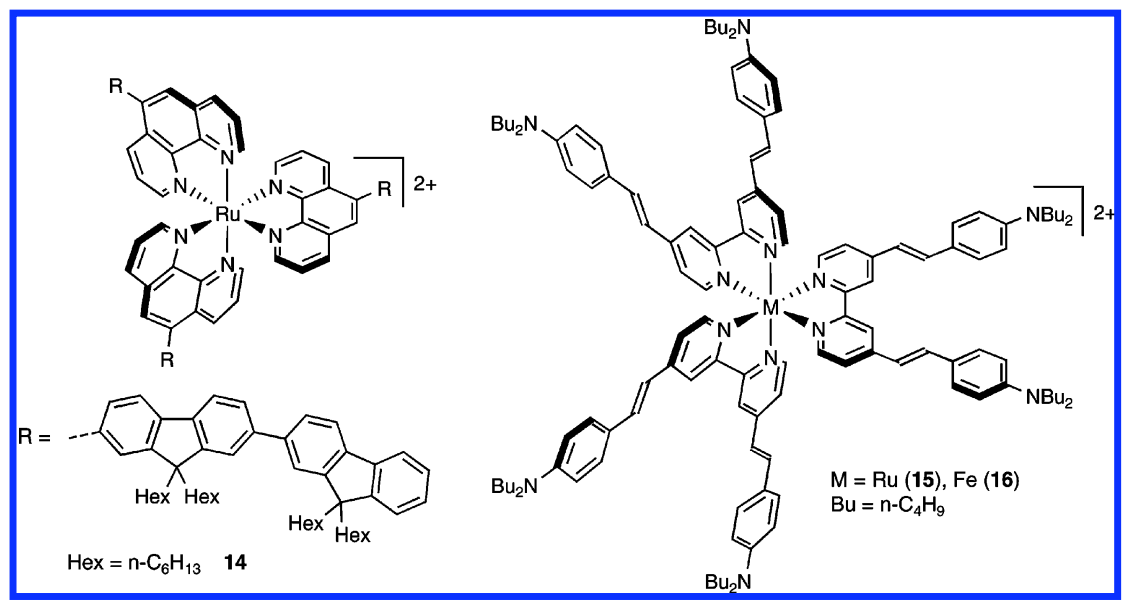

Figure 9. Chemical structures of some complex chromophores investigated for 2PA purposes previously. ${ }^{12}$

7). Such behavior is typical for molecules probed at wavelengths in the vicinity of strong one- or two-photon absorptions. Several trends are evident in the 2PA data. (i) Extending the conjugated systems of the ligands leads to large increases in activity, as expected on the basis of previous reports concerning purely organic chromophores. On moving along the Ru series $\mathbf{1} \rightarrow \mathbf{2}$ $\rightarrow$ 5, a total $\sigma_{2}$ enhancement of ca. 24-fold is observed, while the corresponding increase for the Fe series $\mathbf{7} \rightarrow \mathbf{8} \rightarrow \mathbf{1 1}$ is ca. 115-fold. (ii) Replacing $\mathrm{R}=\mathrm{Me}$ with $\mathrm{R}=\mathrm{Ph}$ causes an approximate doubling of $\sigma_{2}$ in three out of four instances (with 9 providing the exception). However, the 2PA activity of the 2,4-DNPh derivative $\mathbf{1 2}$ is only about half that of $\mathbf{1 0}$. (iii) The previously reported Fe compounds 7 and $\mathbf{9}$ show only very small $\sigma_{2}$ values, while their $\mathrm{Ru}$ analogues $\mathbf{1}$ and $\mathbf{3}$ are much more efficient two-photon absorbers. ${ }^{16}$ However, the data for the new chromophores show that the effect of the metal center becomes less significant as the ligand $\pi$-systems are extended. Hence, the $\mathrm{Ru}$ bbpe-based species $\mathbf{2}$ and $\mathbf{4}$ have $\sigma_{2}$ values about twice those of their $\mathrm{Fe}$ counterparts 8 and 10. The $\mathrm{Me}_{2} \mathrm{bbpb}^{2+}$ complexes in $\mathbf{5}$ and $\mathbf{1 1}$ have indistinguishable 2PA efficiencies, while that of $\mathbf{6}$ is substantially larger than that of $\mathbf{1 3}$.

Our new complex chromophores clearly show much larger $\sigma_{2}$ values in comparison with their previously reported counterparts, but it is also of interest to draw some comparisons with data obtained for other species. Girardot et al. have used twophoton excited luminescence measurements to determine a $\sigma_{2}$ value of $90 \mathrm{GM}$ (at $750 \mathrm{~nm}$ ) for a derivative of $\left[\mathrm{Ru}^{\mathrm{II}}(\mathrm{phen})_{3}\right]^{2+}$ with six fluorenyl substituents (14; Figure 9). ${ }^{12 a}$ The relatively low efficiency in this case is probably attributable to limited $\pi$-conjugation within the ligands due to twisting about the interannular bonds. Nonetheless, a complex related to $\mathbf{1 4}$, with $\sigma_{2}=40 \mathrm{GM}$ at $740 \mathrm{~nm}$, has recently been studied in vitro as a singlet oxygen generator for potential applications in photodynamic cancer therapy. ${ }^{12 \mathrm{c}}$ Some of us have also used the Z-scan approach to derive much larger $\sigma_{2}$ values of $2200 \pm 300$ and $1900 \pm 300 \mathrm{GM}$ (at $765 \mathrm{~nm}$ ) for the complexes $\mathbf{1 5}$ and $\mathbf{1 6}$ (Figure 9), respectively, ${ }^{12 \mathrm{~b}}$ which are of magnitude similar to that reported here for $\mathbf{6}$ (Table 7). It is interesting to note that although 15 contains shorter conjugated systems, its 2PA activity is close to that measured for 6. Comparison with the $\sigma_{2}$ value of $720 \mathrm{GM}$ for $\mathbf{2}$ indicates that replacing an electron-accepting $N$-methylpyridinium group with a di- $n$-butylamino donor enhances the 2PA activity approximately 3-fold. It therefore appears that such tris-chelate complexes give larger 2PA effects when these are associated with directionally opposed MLCT and ILCT transitions (the latter involving strong amino donors as in 14), as opposed to codirectional MLCT and ILCT processes (the latter involving weak pyridyl donors as in 2). However, it is probably inappropriate to speculate further without more extensive studies involving variations in the $\mathrm{Z}$-scan measurement wavelength. Despite the relatively impressive $\sigma_{2}$ values for these new and related complexes, other studies with $\mathrm{Cu}^{\mathrm{I}}$-, $\mathrm{Cd}^{\mathrm{II}}$-, or $\mathrm{Zn}^{\mathrm{II}}$-based systems have afforded considerably larger activities, exceeding even $10^{4} \mathrm{GM}^{52}$ A similarly huge $\sigma_{2}$ value has recently been reported for a dendrimeric nonanuclear $\mathrm{Ru}^{\mathrm{II}} \sigma$-acetylide complex, ${ }^{53}$ and appropriately substituted $\mathrm{Zn}^{\text {II }}$ porphyrin complexes, with their extensive $\pi$-systems, are also among the most active 2PA chromophores known. ${ }^{54}$

\section{Conclusions}

We have synthesized several new bpy-based proligands and used these to extend the family of pyridinium-substituted trischelate complexes of $\mathrm{Ru}^{\mathrm{II}}$ or $\mathrm{Fe}^{\mathrm{II}}$. As the conjugated systems extend, the presence of intense, low-energy ILCT bands becomes more apparent in the electronic absorption spectra. The

(51) The $\beta_{\mathrm{EN}}$ values (10 $10^{-32}$ esu) for (E)-4'-(dimethylamino)- $N$-phenyl-4stilbazolium hexafluorophosphate and its 2,4-dinitrophenyl counterpart are 322.0 and 307.9 , respectively. For the corresponding $(E, E)-1,3-$ butadienyl-containing compounds, the respective values are 602.8 and 532.5. Changing the electron donor to a julolidinyl group gives a similar pattern in the $\beta_{\mathrm{EN}}$ values $\left(10^{-32} \mathrm{esu}\right): 420.4(\mathrm{R}=\mathrm{Ph})$ and 398.0 $(\mathrm{R}=2,4-\mathrm{DNPh})$ for $(E)-N$-R-4-[2-(2,3,6,7-tetrahydro-1H,5H-pyrido[3,2,1-ij]quinolin-9-yl)vinyl]pyridinium hexafluorophosphate and $680.3(\mathrm{R}=\mathrm{Ph})$ and $663.0(\mathrm{R}=2,4-\mathrm{DNPh})$ for the corresponding $(E, E)$-1,3-butadienyl compounds. Note also that the $\beta_{\mathrm{EN}}$ values for these pseudo-1D organic species are about 1 order of magnitude larger than those derived for the 3D tris-chelate complexes (Table 6).

(52) (a) Das, S.; Nag, A.; Goswami, D.; Bharadwaj, P. K. J. Am. Chem. Soc. 2006, 128, 402-403. (b) Jana, A.; Jang, S. Y.; Shin, J.-Y.; Kumar De, A.; Goswami, D.; Kim, D.; Bharadwaj, P. K. Chem. Eur. J. 2008, 14, 10628-10638.

(53) Roberts, R. L.; Schwich, T.; Corkery, T. C.; Cifuentes, M. P.; Green, K. A.; Farmer, J. D.; Low, P. J.; Marder, T. B.; Samoc, M.; Humphrey, M. G. Adv. Mater. 2009, 21, 2318-2322.

(54) (a) Drobizhev, M.; Stepanenko, Y.; Rebane, A.; Wilson, C. J.; Screen, T. E. O.; Anderson, H. L. J. Am. Chem. Soc. 2006, 128, 12432-12433. (b) Kim, K. S.; Noh, S. B.; Katsuda, T.; Ito, S.; Osuka, A.; Kim, D.H. Chem. Commun. 2007, 2479-2481. (c) Collini, E.; Mazzucato, S.; Zerbetto, M.; Ferrante, C.; Bozio, R.; Pizzotti, M.; Tessore, F.; Ugo, R. Chem. Phvs. Lett. 2008, 454, 70-74. (d) Odom, S. A.; et al. J. Am. Chem. Soc. 2009, 131, 7510-7511. (e) Webster, S.; Odom, S. A.; Padilha, L. A.; Przhonska, O. V.; Peceli, D.; Hu, H.-H.; Nootz, G.; Kachkovski, A. D.; Matichak, J.; Barlow, S.; Anderson, H. L.; Marder, S. R.; Hagan, D. J.; Van Stryland, E. W. J. Phvs. Chem. B 2009, 113, 14854-14867. 
MLCT bands show red shifts on extending the $\pi$-conjugation, increasing the electron acceptor strength of the pyridinium groups, and/or replacing $\mathrm{Ru}$ with Fe. Cyclic voltammetry reveals reversible $\mathrm{M}^{\mathrm{III} / \mathrm{II}}$ waves, but generally irreversible ligand-based reductions. The $\beta$ responses determined by via HRS measurements at 800 and $1064 \mathrm{~nm}$ are very large (up to $5600 \times 10^{-30}$ esu), but are strongly enhanced by resonance. Stark spectroscopic studies on the MLCT bands lead to estimated $\beta_{0}$ values as high as ca. $600 \times 10^{-30}$ esu in one instance, which represent lower limits because the contributions associated with the ILCT bands are not taken into account in most cases. For the two compounds where the ILCT contributions are also amenable to Stark analysis, potentially record $\beta_{0}$ values as high as ca. $10^{-27}$ esu are determined. These indirectly derived NLO responses generally increase on extending the $\pi$-conjugation and increasing the electron-accepting strength of the ligands, and are much larger than those determined previously for related complexes. ${ }^{15}$ However, no clear superiority of either $\mathrm{Ru}$ or $\mathrm{Fe}$ is evident. The measured dipole moment changes can be as large as ca. 34 $\mathrm{D}$ and show the expected dependence on conjugation path length. $Z$-scan measurements at $750 \mathrm{~nm}$ reveal high $\sigma_{2}$ values of up to $2500 \mathrm{GM}$; these are slightly larger than or similar to those measured for related electron-donor-substituted complexes, ${ }^{12 \mathrm{~b}}$ but are as much as an order of magnitude higher than the activities we have determined previously for pyridinium derivatives. ${ }^{16}$ The new chromophores reported are therefore relatively rare examples of species that combine unusually high quadratic and cubic NLO effects with potentially redoxswitchable transition metal centers.

Acknowledgment. We thank the EPSRC for support (grants EP/D070732 and EP/E000738) and also the Fund for Scientific Research-Flanders (FWO-V, G.0312.08), the University of Leuven (GOA/2006/3), the NSF (grant CHE-0802907, 'Powering the Planet: an NSF Center for Chemical Innovation') and the Foundation for Polish Science. I.A. is a postdoctoral fellow of the FWO-V and M.S. is a Laureate of the FNP Welcome programme.

Supporting Information Available: Crystallographic information in CIF format; Figures S1-S3; complete ref 54d (PDF). This material is available free of charge via the Internet at http:// pubs.acs.org.

JA910538S 